National Water-Quality Assessment Program

\title{
Linking Urbanization to the Biological Condition Gradient (BCG) for Stream Ecosystems in the Northeastern United States Using a Bayesian Network Approach
}

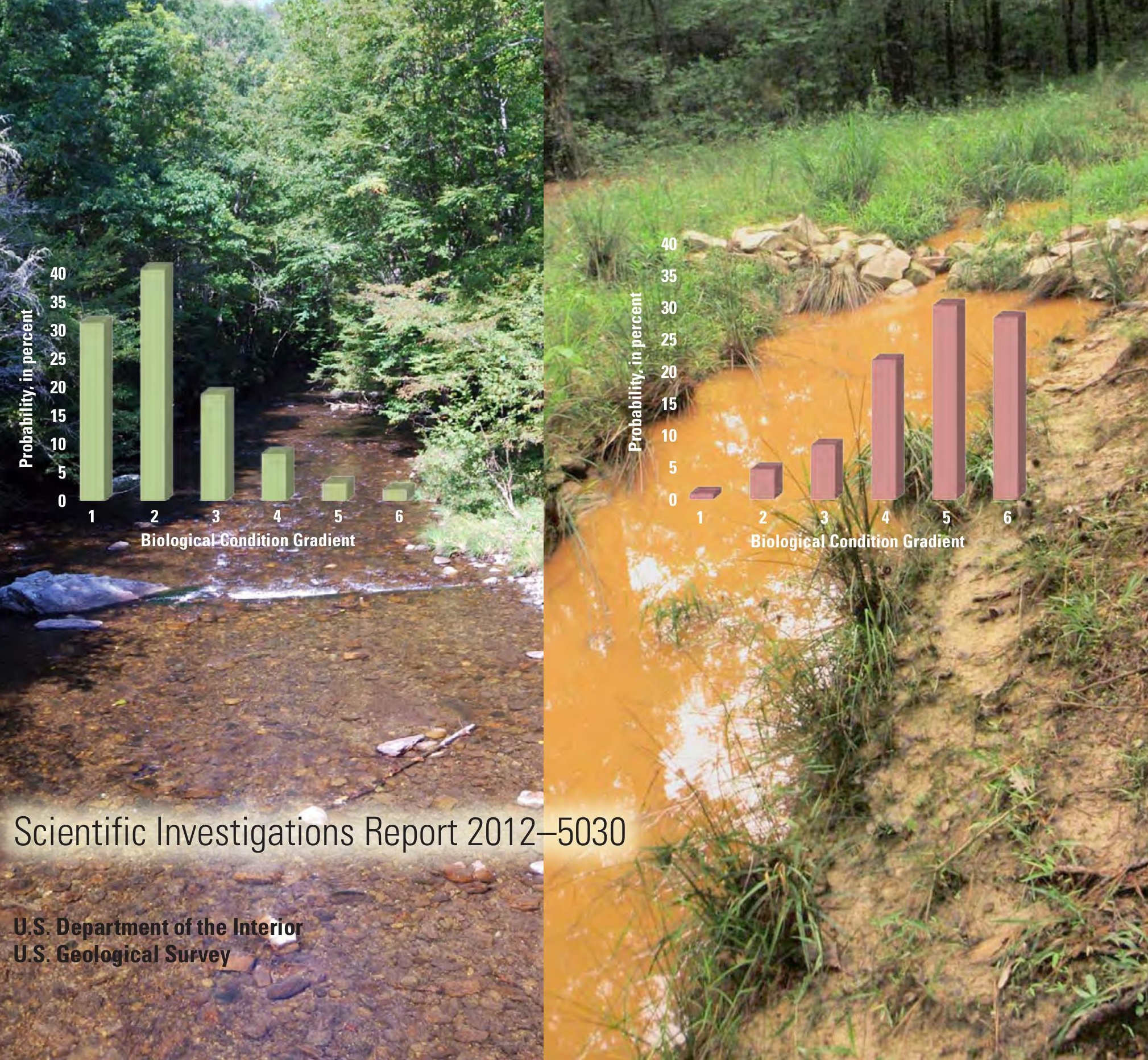


Cover. Left photograph-Example of a stream with low levels of urban stress, which is more likely to have biological condition in BCG tiers 1 or 2 as shown by the superimposed probability distribution in green (Thomas F. Cuffney, USGS, September 2011).

Right photograph-Example of a stream with high levels of urban stress, which is more likely to have biological condition in BCG tiers 5 or 6 as shown by the superimposed probability distribution in red (Michelle Moorman, USGS, April 2011). 


\section{Linking Urbanization to the Biological Condition Gradient (BCG) for Stream Ecosystems in the Northeastern United States Using a Bayesian Network Approach}

By Roxolana Kashuba, Gerard McMahon, Thomas F. Cuffney, Song Qian, Kenneth Reckhow, Jeroen Gerritsen, and Susan Davies

Prepared in cooperation with Duke University

Scientific Investigations Report 2012-5030 


\title{
U.S. Department of the Interior \\ KEN SALAZAR, Secretary \\ U.S. Geological Survey \\ Marcia K. McNutt, Director
}

\author{
U.S. Geological Survey, Reston, Virginia: 2012
}

For more information on the USGS - the Federal source for science about the Earth, its natural and living resources, natural hazards, and the environment, visit http://Www.usgs.gov or call 1-888-ASK-USGS.

For an overview of USGS information products, including maps, imagery, and publications, visit $h t t p: / / w w w . u s g s . g o v / p u b p r o d$

To order this and other USGS information products, visit http://store.usgs.gov

Any use of trade, product, or firm names is for descriptive purposes only and does not imply endorsement by the U.S. Government.

Although this report is in the public domain, permission must be secured from the individual copyright owners to reproduce any copyrighted materials contained within this report.

Suggested citation:

Kashuba, Roxolana, McMahon, Gerard, Cuffney, T.F., Qian, Song, Reckhow, Kenneth, Gerritsen, Jeroen, and Davies, Susan, 2012, Linking urbanization to the Biological Condition Gradient (BCG) for stream ecosystems in the Northeastern United States using a Bayesian network approach: U.S. Geological Survey Scientific Investigations Report 2012-5030, 48 p.

(Available online at http://pubs.usgs.gov/sir/2012/5030/ 


\section{Acknowledgments}

The authors would like to thank David Courtemanch and Thomas Danielson of the Maine Department of Environmental Protection for great investments of time and effort toward Biological Condition Gradient (BCG) discussion and extensive expert elicitation development exercises.

James Coles and Nathaniel Plant of the U.S. Geological Survey provided discerning technical reviews that improved this report substantially.

Finally, we owe all expert teams a large measure of gratitude for agreeing to share their considerable expertise and for their openness to new ideas and new ways of thinking. 



\section{Contents}

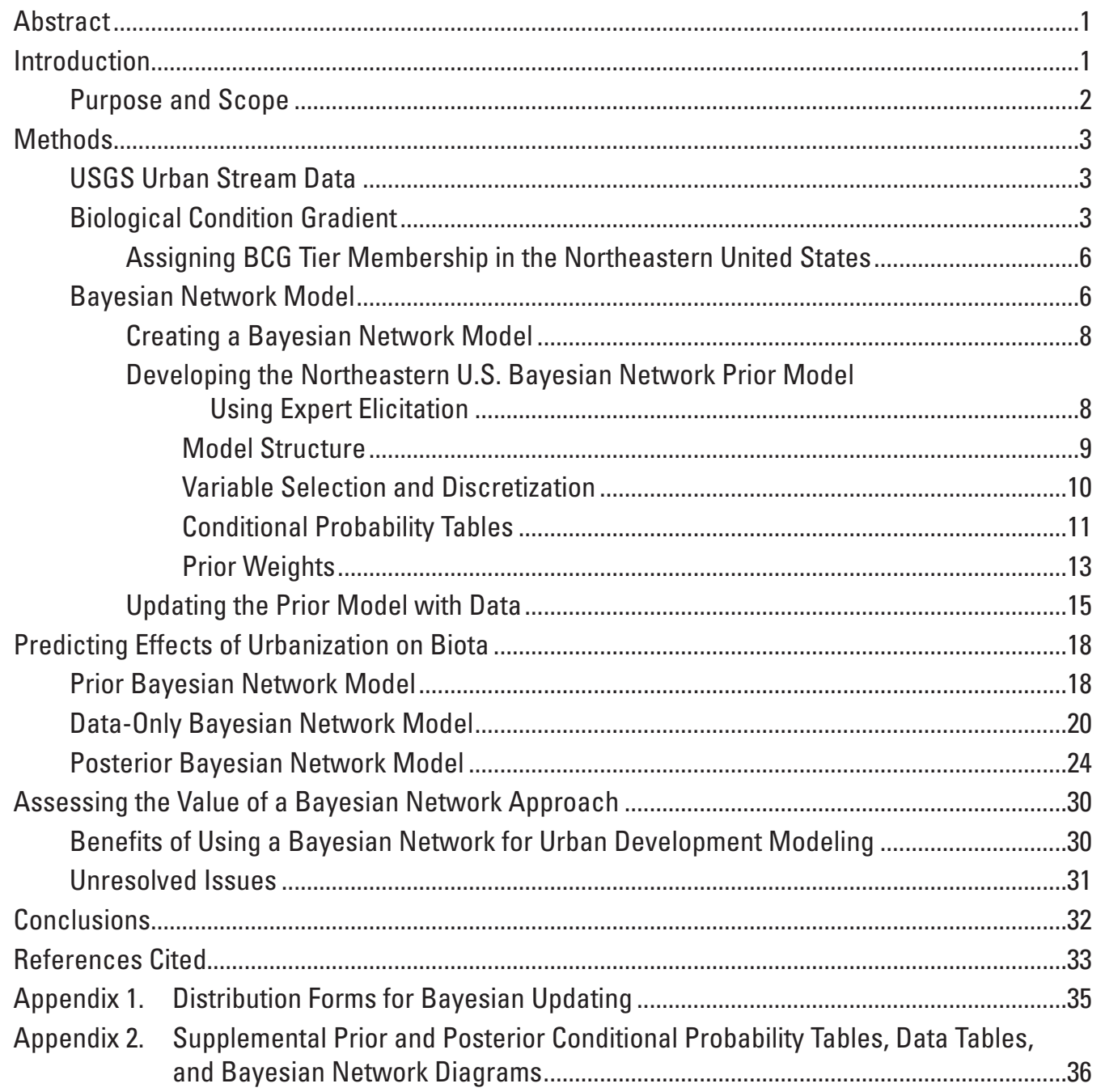




\section{Sidebars}

1. Comparing Stream Biological Conditions Across Regions................................................5

2. How Bayesian Updating Works............................................................................................16

Simple Example with Single Probability Parameter....................................................16

Bayesian Updating for Multiple Probability Parameters within a Bayesian Network CPT Row

\section{Figures}

1. Conceptual diagram of the Effects of Urbanization on Stream Ecosystems (EUSE) National Water-Quality Assessment (NAWQA) Program study ....

2. Map showing locations of the nine metropolitan regions and surrounding areas of the Effects of Urbanization on Stream Ecosystems (EUSE) studies ................................

3. Graph showing Biological Condition Gradient (BCG) tiers relative to increasing stressor gradient......................................................................................................... 4

4. Graph showing Midwest versus Appalachian stream conditions ....................................5

5. Directed acyclic graph (DAG) showing a simple Bayesian network with four nodes and four arrows

6. Example of simple conditional probability tables (CPT) ....................................................

7. Diagram showing parsimonious Northeast Bayesian network model structure.............10

8. Graph showing Bayesian updating of hypothetical, generic parameter $\theta_{i} \ldots \ldots \ldots \ldots \ldots \ldots . . . . .16$

9. Charts showing Bayesian updating of parameters $\theta_{1}, \theta_{2}, \theta_{3}$, within a conditional probability table (CPT) row in a Bayesian network.

10. Diagram showing prior predictive probabilities of occurrence for each level of each node under low urban development.

11. Diagram showing prior predictive probabilities of occurrence for each level of each node under high urban development.

12. Diagram showing data-only predictive probabilities of occurrence for each level of each node under low urban development.

13. Diagram showing data-only predictive probabilities of occurrence for each level of each node under high urban development.

14. Diagram showing posterior predictive probabilities of occurrence for each level of each node given an observation of high urban land cover and no management interventions.

15. Diagram showing posterior predictive probabilities of occurrence for each level of each node under causal inference: Managing flashiness to medium, while still observing high urban land cover

16. Diagram showing posterior predictive probabilities of occurrence for each level of each node under causal inference: Managing specific conductance to low, while still observing high urban land cover

17. Diagram showing posterior predictive probabilities of occurrence for each level of each node under causal inference: Managing flashiness to medium and specific conductance to low, while still observing high urban land cover. 


\section{Appendix Figures}

2-1. Prior Northeast Bayesian network ...........................................................................................

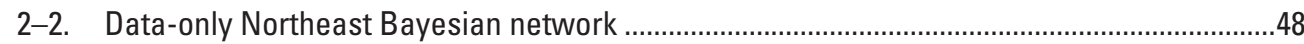

\section{Tables}

1. Biological Condition Gradient (BCG) tier and attribute definitions...................................

2. Northeastern U.S. urbanization effects model expert teams ............................................

3. Nodes and variables chosen by experts to represent major system components in the Northeastern U.S. Bayesian network model........................................................11

4. "Hydrology" node prior conditional probability table, data table, and posterior

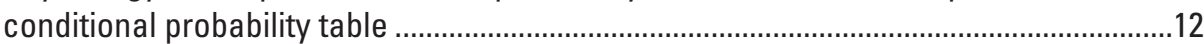

5. Probability range prior weight elicitation results for all child state probabilities in each of two randomly selected rows from each conditional probability table ...........14

6. Driver causal interventions most likely to improve Biological Condition Gradient (BCG) tier achievement likelihood given unobserved urban land cover state.................29

7. Charts showing excerpts from macroinvertebrate metric prior expert-elicited conditional probability tables

\section{Appendix Tables}

2-1. "Habitat" node prior conditional probability table and prior weight $\left(\alpha_{0}\right)$, data table, and posterior conditional probability table and posterior weights $\left(\alpha_{0}+n\right)$.

2-2. "Water quality" node prior conditional probability table and prior weight $\left(\alpha_{0}\right)$, data table, and posterior conditional probability table and posterior weights $\left(\alpha_{0}+n\right) \ldots . .37$

2-3. "Generic richness" node prior conditional probability table and prior weight $\left(\alpha_{0}\right)$, data table, and posterior conditional probability table and posterior weights $\left(\alpha_{0}+n\right) \ldots . .38$

2-4. "Filter feeder relative abundance" node prior conditional probability table and prior weight $\left(\alpha_{0}\right)$, data table, and posterior conditional probability table and posterior weights $\left(\alpha_{0}+n\right)$.

2-5. "P+E relative abundance" node prior conditional probability table and prior weight $\left(\alpha_{0}\right)$, data table, and posterior conditional probability table and posterior weights $\left(\alpha_{0}+n\right)$

2-6. "BCG" node prior conditional probability table and prior weight $\left(\alpha_{0}\right)$, data table, and posterior conditional probability table and posterior weights $\left(\alpha_{0}+n\right)$. 


\section{Abbreviations}

$\begin{array}{ll}\text { BCG } & \text { Biological Condition Gradient } \\ \text { BMP } & \text { Best Management Practices } \\ \text { CART } & \text { Classification and regression tree } \\ \text { CPT } & \text { Conditional probability tables } \\ \text { DAG } & \text { Directed acyclic graph } \\ \text { EPT } & \text { Ephemeroptera (mayflies), Plecoptera (stoneflies), and } \\ & \text { Trichoptera (caddisflies) } \\ \text { EUSE } & \text { Effects of Urbanization on Stream Ecosystems } \\ \text { MCMC } & \text { Markov Chain Monte Carlo } \\ \text { NAWQA } & \text { National Water-Quality Assessment } \\ \text { NECB } & \text { New England Coastal Basins } \\ \text { NEWS } & \text { New England Wadeable Streams } \\ \text { OLR } & \text { Ordered logistic regression } \\ \text { P+E } & \text { Plecoptera and Ephemeroptera } \\ \text { USEPA } & \text { U.S. Environmental Protection Agency } \\ \text { USGS } & \text { U.S. Geological Survey }\end{array}$




\title{
Linking Urbanization to the Biological Condition Gradient (BCG) for Stream Ecosystems in the Northeastern United States Using a Bayesian Network Approach
}

\author{
By Roxolana Kashuba, ${ }^{1}$ Gerard McMahon, ${ }^{1}$ Thomas F. Cuffney, ${ }^{1}$ Song Oian, ${ }^{2}$ Kenneth Reckhow, ${ }^{2}$ \\ Jeroen Gerritsen, ${ }^{3}$ and Susan Davies ${ }^{4}$
}

\section{Abstract}

Urban development alters important physical, chemical, and biological processes that define urban stream ecosystems. An approach was developed for quantifying the effects of these processes on aquatic biota, and then linking those effects to endpoints that can be used for environmental management. These complex, interacting systems are challenging to model from a scientific standpoint. A desirable model clearly shows the system, simulates the interactions, and ultimately predicts results of management actions. Traditional regression techniques that calculate empirical relations between pairs of environmental factors do not capture the interconnected web of multiple stressors, but urban development effects are not yet understood at the detailed scales required to make mechanistic modeling approaches feasible. Therefore, in contrast to a fully deterministic or fully statistical modeling approach, a Bayesian network model provides a hybrid approach that can be used to represent known general associations between variables while acknowledging uncertainty in predicted outcomes. It does so by quantifying an expert-elicited network of probabilistic relations between variables. Advantages of this modeling approach include (1) flexibility in accommodating many model specifications and information types; (2) efficiency in storing and manipulating complex information, and to parameterize; and (3) transparency in describing the relations using nodes and arrows and in describing uncertainties with discrete probability distributions for each variable.

In realization of the aforementioned advantages, a Bayesian network model was constructed to characterize the effect of urban development on aquatic macroinvertebrate stream communities through three simultaneous, interacting ecological pathways affecting stream hydrology, habitat, and

\footnotetext{
${ }^{1}$ U.S. Geological Survey.

${ }^{2}$ Nicholas School of the Environment, Duke University, Durham, N.C.

${ }^{3}$ Tetra Tech, Inc., Owings Mills, Md.

${ }^{4}$ Maine Department of Environmental Protection.
}

water quality across watersheds in the Northeastern United States. This model incorporates both empirical data and expert knowledge to calculate the probabilities of attaining desired aquatic ecosystem conditions under different urban stress levels, environmental conditions, and management options. Ecosystem conditions are characterized in terms of standardized Biological Condition Gradient (BCG) management endpoints. This approach to evaluating urban developmentinduced perturbations in watersheds integrates statistical and mechanistic perspectives, different information sources, and several ecological processes into a comprehensive description of the system that can be used to support decision making. The completed model can be used to infer which management actions would lead to the highest likelihood of desired BCG tier achievement. For example, if best management practices (BMP) were implemented in a highly urbanized watershed to reduce flashiness to medium levels and specific conductance to low levels, the stream would have a 70-percent chance of achieving BCG Tier 3 or better, relative to a 24-percent achievement likelihood for unmanaged high urban land cover. Results are reported probabilistically to account for modeling uncertainty that is inherent in sources such as natural variability and model simplification error.

\section{Introduction}

Urban development affects physical, chemical, and biological processes that shape the condition of stream ecosystems (fig. 1). Impervious surfaces disrupt patterns of water movement (Klein, 1979; Poff and others, 1997; McMahon and others, 2003), and the resulting flashy streamflows can alter the physical habitat of streams, as evident in channel scouring and incision (Booth, 1990), sedimentation (Wolman and Schick, 1967; Trimble, 1997), floodplain disconnection (Haase, 2003), and the removal of riparian habitat. Urban storm runoff also contains anthropogenic contaminants, such as industrial chemicals, pesticides, and human waste (Van Metre and others, 2000; Beck, 2005). Habitat and stream 

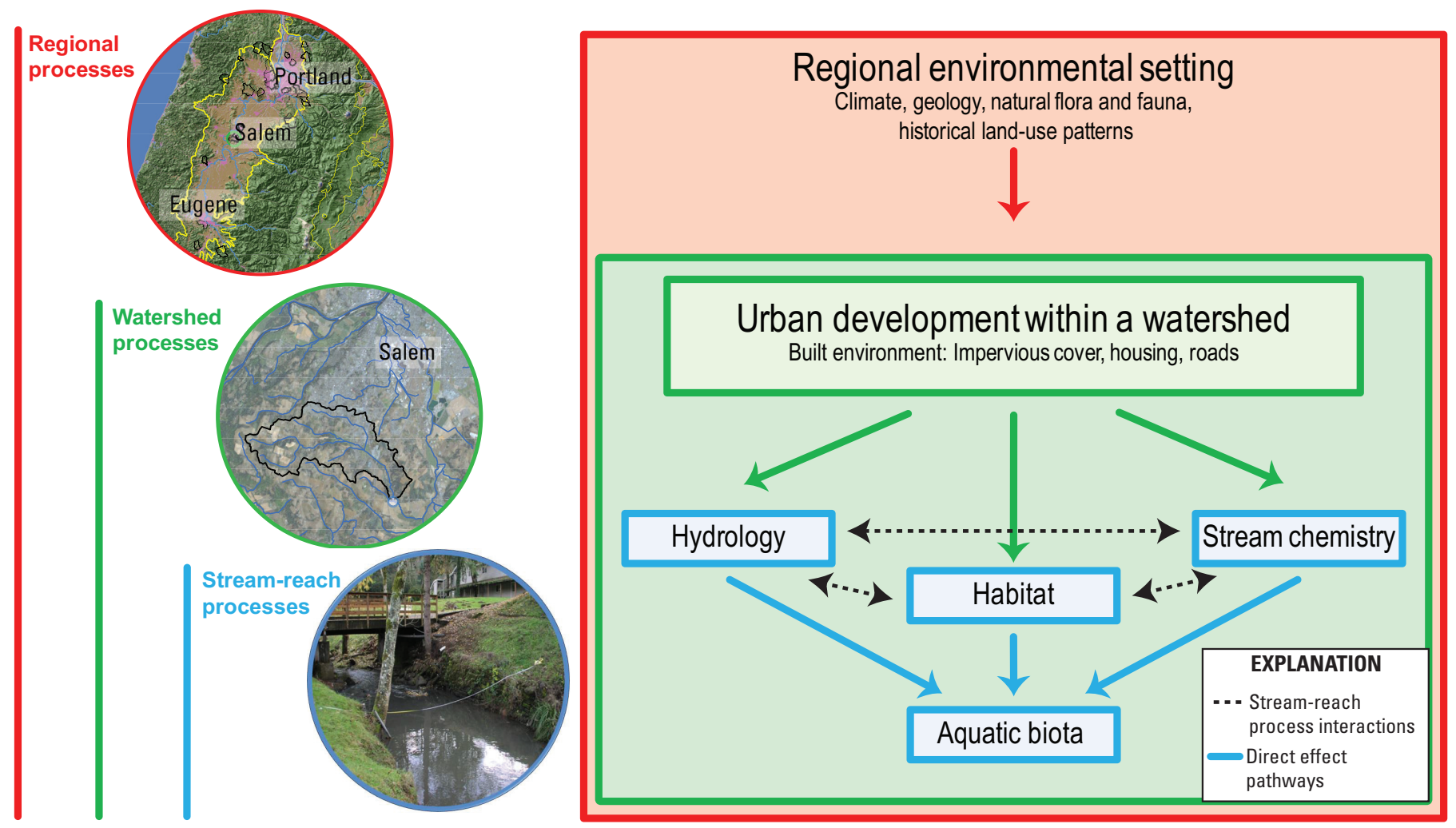

Figure 1. Conceptual diagram of the Effects of Urbanization on Stream Ecosystems (EUSE) National Water-Quality Assessment (NAWOA) Program study. Urban stream ecosystems include processes operating at regional, watershed, and stream-reach scales.

chemistry changes can destabilize stream-nutrient, energy, and temperature cycles (Jacobson, 2001; Sprague and others, 2006). This interacting suite of physical and chemical stream factors serves as stressors and can degrade the condition of aquatic biota (Jones and Clark, 1987; Yoder and others, 1999). Understanding how a stream ecosystem will respond to management actions designed to mitigate the effects of urban development first requires understanding how processes are linked across spatial scales and how they can interact within a given level (indicated in fig. 1 by dashed lines for streamreach processes).

As used to understand and quantify the effects of urban development on stream macroinvertebrates, traditional regression techniques that only calculate empirical relations between pairs of environmental factors (Cuffney and others, 2010) do not account for the complex interactions between these multiple stressors. In addition, regression models do not incorporate available and information-rich expert knowledge, nor do they systematically account for uncertainty associated with system complexity, imperfect scientific understanding, and environmental variability.

To address the aforementioned concerns, the U.S. Geological Survey (USGS), in cooperation with Duke University, developed a Bayesian network approach to model the system of interacting stressors associated with urbanization and their effects on stream biota. This study advances the USGS mission by creating a tool that can be used to assess potential outcomes of different biological resource management strategies including an explicit incorporation and representation of uncertainty associated with those decisions. The study and refinement of such tools will enable the public to enjoy healthy aquatic resources by identifying sustainable urbanization practices that minimize negative effects on the environment.

\section{Purpose and Scope}

This report presents a Bayesian network modeling approach to (1) understand the relation between urban development, reach-scale factors (stressors) that are affected by urban development, and the condition of aquatic biota; and (2) provide information that can be used to manage water quality. A Bayesian network is a probabilistic, graphical model that quantifies a network of relations between variables. This type of model is flexible in accommodating many model structural configurations and input information types, efficient in storing and manipulating complex information, efficient to parameterize, and transparent in the relations it describes through the use of nodes and arrows. In addition, uncertainty is clearly represented as discrete probability distributions across all possible values of each modeled variable. 
To link scientific understanding to management decision making, the Biological Condition Gradient (BCG) (Davies and Jackson, 2006) framework is used as the response variable, or endpoint, for the Bayesian network model. The BCG defines six tiers of biological community condition that can be used by managers as reference points for assessing stream health and determining management actions. The use of Bayesian network models to predict BCG tier membership for individual streams supports management decision-making objectives, including

1. Providing objective and replicable characterizations of the relation between urban development and endpoints for stream health that can be linked to management actions;

2. Describing and quantifying how changes in stressors can lead to changes in a biological-resource endpoint, characterized by the BCG; and

3. Supporting management decision making by calculating the probability of attaining aquatic ecosystem endpoints (BCG tiers) assuming different levels of urban stressors, environmental conditions, and ultimately, management options.

This model provides a realistic and applied method for resource managers to understand the multiple stressors affecting biological communities and evaluate alternative solutions that make sense from an ecological perspective.

The scope of this report is limited to a description of a parsimonious eight-node Bayesian network model constructed using the New England Coastal Basins (NECB) subset of the USGS Effects of Urbanization on Stream Ecosystems (EUSE) data (Cuffney and Falcone, 2008). This report excludes discussion of the original Bayesian network urbanization modeling methods development process and justification, which focused on the Southeast United States (Kashuba, 2010). The focus of this report centers on model application and, as such, excludes the details of statistical methods innovations in the parameterization and understanding of Bayesian network modeling.

\section{Methods}

This section describes data-collection design, biological endpoint standardization and assignment, and the process involved in building and updating a Bayesian network model.

\section{USGS Urban Stream Data}

A set of nine watershed studies designed to investigate the Effects of Urbanization on Stream Ecosystems (EUSE) was conducted in major U.S. metropolitan areas during 1999-2004 as part of the USGS National Water-Quality Assessment (NAWQA) Program (fig. 2; Tate and others, 2005; Giddings and others, 2009). These studies assessed the

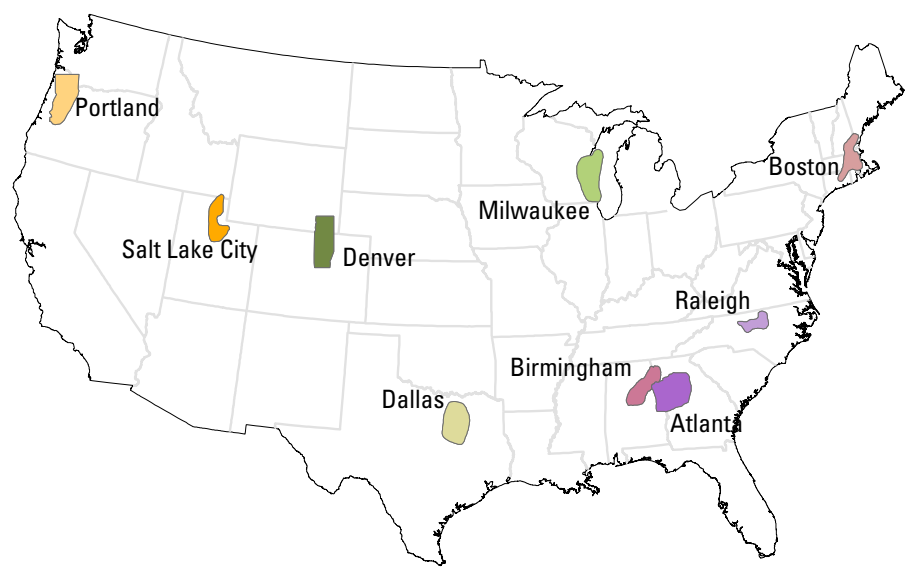

Figure 2. Locations of the nine metropolitan regions and surrounding areas of the Effects of Urbanization on Stream Ecosystems (EUSE) studies.

physical, chemical, and biological conditions of streams across a range of urban development with a common design that used standardized measures of urban intensity (Cuffney and Falcone, 2008), methods of collection and processing samples, and techniques for data analysis (Fitzpatrick and others, 1998; Moulton and others, 2002). Within each of the nine metropolitan areas, data were collected at approximately 30 similarly sized watersheds. By substituting space for time, the EUSE studies quantified many environmental and biological stream changes that occur as urban development progresses within a watershed. The EUSE study conducted in the Northeastern United States, which included the Boston metropolitan area (fig. 2), was designated the NECB EUSE study; data from this study were used in the modeling effort described herein. New England was chosen as the area of focus to take advantage of the regional expertise of local biologists who were instrumental in developing the BCG concept (Davies and Jackson, 2006).

\section{Biological Condition Gradient}

The BCG provides a framework for assessing stream health in terms of the condition of resident aquatic biota (fig. 3; table 1; Davies and Jackson, 2006). The biological condition of a stream is reported in terms of six tiers that represent biological condition endpoints ranging from natural (Tier 1) to highly disturbed (Tier 6); these endpoints are suitable for determining whether Clean Water Act water-quality standards are attained.

The assignment of a stream to a BCG tier relies on the exercise of scientific judgment, dependent on regional expertise. Given a taxonomic group of interest (for example, macroinvertebrates), experts determine the community characteristics associated with each of 10 BCG attribute classes that are appropriate for a given region of the country (table 1). The overall assignment of a stream to a BCG tier is done by 


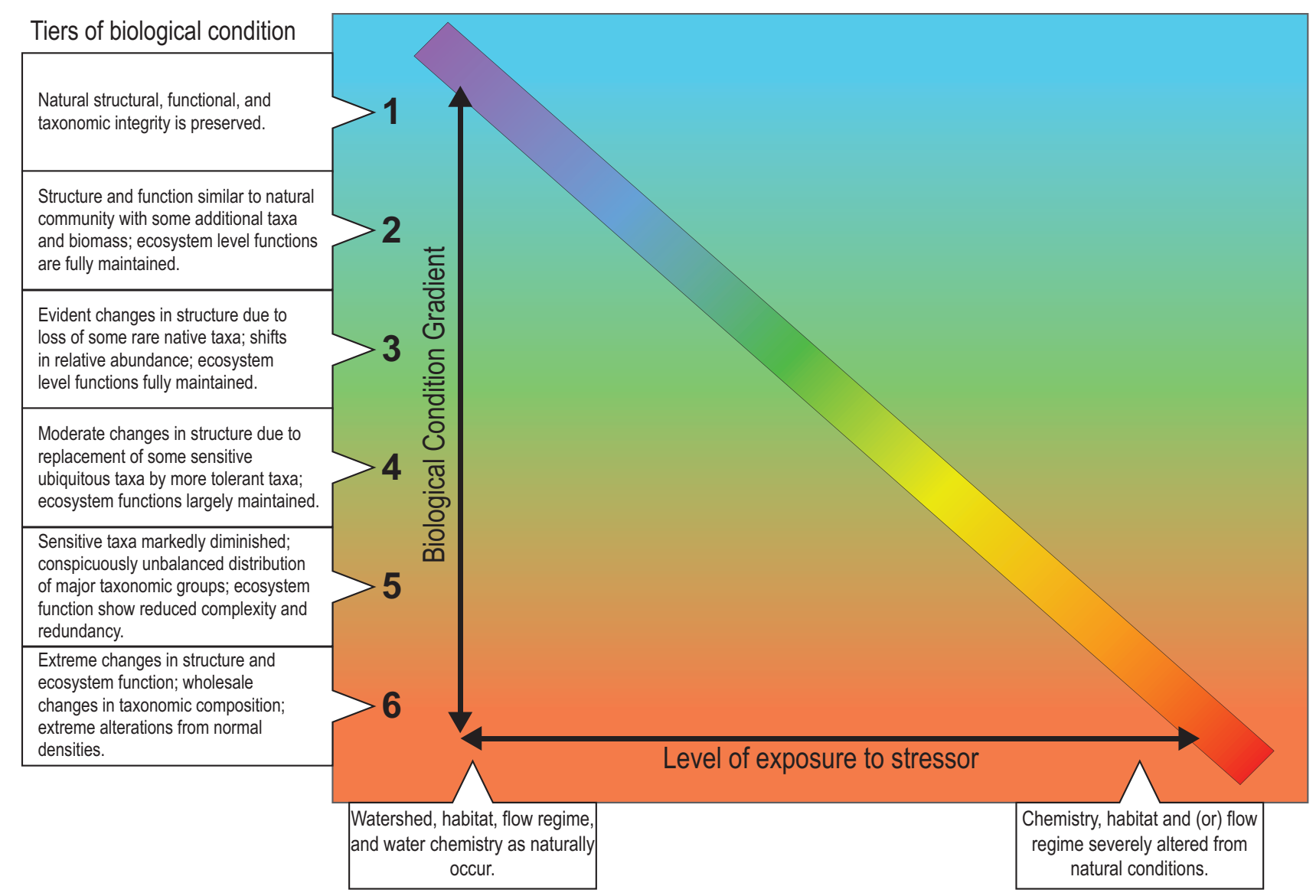

Figure 3. Biological Condition Gradient (BCG) tiers relative to increasing stressor gradient (modified from Davies and Jackson, 2006). The six BCG tiers are defined in terms of biotic community structure and ecosystem function as listed in table 1.

Table 1. Biological Condition Gradient (BCG) tier and attribute definitions.

[From Davies and Jackson (2006). The six BCG tiers are expressed graphically across increasing exposure to stressors in figure 3]

\begin{tabular}{cll}
\hline Tier & \multicolumn{1}{c}{ Structure of biotic community } & \multicolumn{1}{c}{ Ecosystem function } \\
\hline 1 & Natural or native condition & Natural or native condition \\
2 & Minimal changes & Minimal changes \\
3 & Evident changes & Minimal changes \\
4 & Moderate changes & Minimal changes \\
5 & Major changes & Moderate changes \\
6 & Severe changes & Major loss \\
\hline Attribute & & \\
\hline I & Historically documented, sensitive, long-lived or regionally endemic taxa \\
II & Sensitive-rare taxa & \\
III & Sensitive-ubiquitous taxa & \\
IV & Taxa of intermediate tolerance & \\
V & Tolerant taxa & \\
VI & Nonnative or intentionally introduced taxa & \\
VII & Organism condition & \\
VIII & Ecosystem function & \\
IX & Spatial and temporal extent of detrimental effects \\
X & Ecosystem connectance & \\
\hline
\end{tabular}




\section{Sidebar 1. Comparing Stream Biological Conditions Across Regions}

A major challenge in assessing relative biological conditions for streams in different parts of the country is setting expectations for the assessed sites that account for regional differences in the best available, or "reference," conditions of aquatic biota (Herlihy and others, 2008). Macroinvertebrate samples from a U.S. Environmental Protection Agency (USEPA) study of the Temperate Plains region, which includes extensive agricultural lands in the eastern Dakotas, through lowa, Minnesota, Missouri, Kansas, and Nebraska, to Indiana and Ohio, can be interpreted in a way that indicates that 62 percent of the stream length in this region was in good or fair condition. In contrast, macroinvertebrate sample data from the Northern Appalachians region, which includes all the New England states, most of New York, the northern half of Pennsylvania, and northeastern Ohio, can be interpreted in a way that indicates that only 28 percent of the stream length was in good or fair condition. A conclusion that could be drawn from these data, collected as part of the USEPA Wadeable Streams Assessment, is that the overall biological condition of streams in the Midwestern agricultural region is better than the condition of streams in the largely forested Appalachian region (fig. 4; U.S. Environmental Protection Agency, 2006).

A likely reason for the larger percentage of good and fair stream reaches in the Temperate Plains in comparison to the Northern Appalachians is the relative difference in the observed biological condition of the reference streams in the two regions. Reference streams were selected on the basis of the "best available conditions," those conditions generally present in undisturbed watersheds. Macroinvertebrate communities in these streams typically have more sensitive species than streams in watersheds that have been subject to some degree of human disturbance. However, much of the Temperate Plains has been altered by human disturbance, such as agricultural activity, and reference streams meeting the criterion of "best available conditions" had relatively fewer sensitive species than did reference streams in the Northern Appalachians.

In essence, the regional difference in disturbance led to unequal

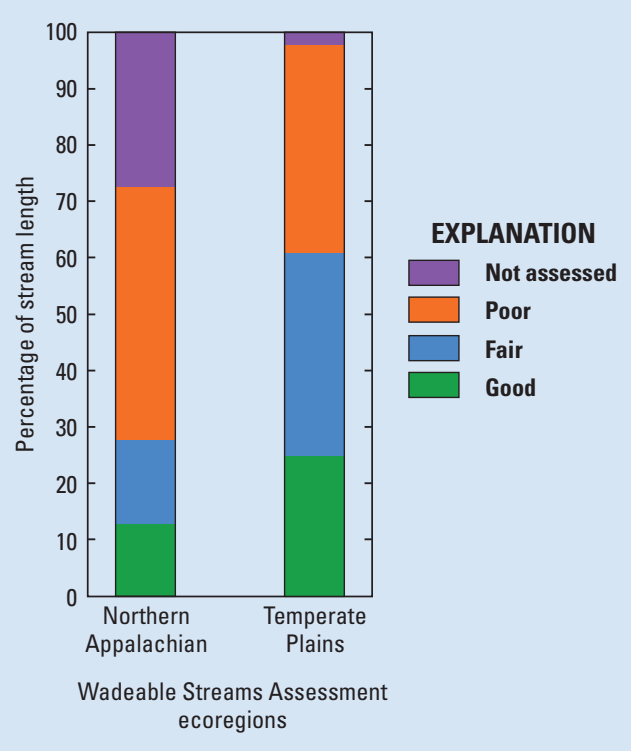

Figure 4. Midwest versus Appalachian stream conditions. starting points for comparing streams in the Temperate Plains with those in the Northern Appalachians. In terms of the Effects of Urbanization on Stream Ecosystems (EUSE) design, the relatively limited range of biological conditions in the Temperate Plains streams, including reference streams, resulted in a "truncated gradient" in comparison to the Northern Appalachian streams, where reference streams were more likely to have sensitive species. Therefore, for a given level of disturbance in a watershed (that is, moving away from reference conditions) a stream in the Northern Appalachian region would be likely to show greater departure from reference conditions compared to a stream in the Temperate Plains region. Additionally, a truncated gradient for the Temperate Plains region is consistent with the EUSE finding that agricultural land use in watersheds in the Milwaukee, Dallas, and Denver study areas had probably led to a reduction in the number of sensitive species in streams prior to urban development (Kashuba and others, 2010).

An issue of potential concern for management in using least-disturbed streams to define "reference conditions" is that the qualities of a healthy stream can vary greatly among regions. In cases where assessments of stream health are based on regionally distinct reference biological communities, they are often difficult to compare across regions. This situation can occur, for example, when tolerant species dominate the biological community in the least-disturbed streams of a region because sensitive species have been lost from prior land-use disturbances. One solution that addresses this inconsistency is the use of a Biological Condition Gradient (BCG) (Davies and Jackson, 2006). The BCG provides a frame of reference for assessing stream biological conditions that can be applied in all parts of the United States. Best available stream conditions have reference conditions in BCG Tiers 1 or 2 in parts of the country that include high-quality biological communities. Other parts of the country, with degraded biological communities, may have reference conditions in BCG Tiers 3 or 4 . Informative cross-regional comparisons can only be made when they are based on a common frame of reference. 
characterizing the organisms found in a biological sample in terms of these attributes (table 1). This characterization can be done by designating the appropriate attribute category for individual organisms (for example, sensitive rare taxa) or using metrics that provide summaries of the community composition. Experts determine the set of metrics that are best suited to represent the attributes for a given region and the appropriate range of metric values associated with each tier. A stream is assigned by experts to a BCG tier by weighing evidence from specific organisms and summary metrics. For example, a site with many sensitive, rare taxa belonging to Attribute II and few tolerant taxa belonging to Attribute $\mathrm{V}$ may be assigned a BCG Tier 2, reflecting a judgment that the overall structure of that site's biotic community differs minimally from the natural condition.

Implementation of a BCG framework allows waterquality managers to identify and protect waters of exceptional quality and detect incremental improvement or degradation in a stream at a finer scale than currently practiced. Managers can also use the BCG to set realistically attainable restoration goals, diagnose problems and causes with increased detection sensitivity, and find problems early and correct them while they are still local (Davies and Jackson, 2006). The BCG attempts to facilitate comparisons among regions by taking into account regional differences in the distribution of fauna and differences in methods of data collection and parameterization used by State and Federal agencies that monitor biological conditions (Davies and Jackson, 2006) (sidebar 1).

\section{Assigning BCG Tier Membership in the Northeastern United States}

In the Northeastern United States, substantial effort has been exerted to derive expert consensus on the classification of macroinvertebrate taxa into BCG attributes associated with community structure (Attributes I-VI; table 1). Based on extensive previous work conducted in the Northeastern United States by the U.S. Environmental Protection Agency (USEPA) (Snook and others, 2007; Yoder and Barbour, 2009), Tetra Tech Inc. (Gerritsen, 2008), and the State of Maine (Shelton and Blocksom, 2004), some 700 taxa sampled in the EUSE NECB data, hereafter referred to as EUSE Northeast data, were classified by attribute characteristics. These characteristics relate to the relative sensitivity of specific macroinvertebrates in the Northeast, but the same taxa would need to be re-classified for other ecoregions. For example, caddisfly Oecetis is classified as a genus of intermediate tolerance (Attribute IV) in the Northeast but as a sensitive-ubiquitous genus (Attribute III) in the Midwest (Barbour and others, 1999). BCG Attributes VII-X describe ecological function and are much more challenging to measure than those involving community structure. Therefore, these attributes have not yet been characterized in implementations of the BCG framework (Gerritsen, 2008).

Once EUSE Northeast taxa were classified, biological experts were given 30 spreadsheets (one for each NECB stream sampled) that characterized each site's macroinvertebrate community with a list of taxa found at that site, sorted and color-coded by attribute. In addition, spreadsheets also contained 20 standard macroinvertebrate metrics describing the richness and abundance of common indicator groups of taxa (Ephemeroptera, Plecoptera, and Trichoptera (EPT), Chironomidae, Oligochaeta, and so forth). Using their long-term career experience in judging biological condition, experts used this information to assign one BCG tier (Tier 1-6) to each NECB site. Tiers were first assigned individually, and final assignments were reached through expert group discussion and consensus. These assignments were then considered part of the EUSE Northeast dataset.

To operationalize the BCG approach, results from implementing the BCG framework need to be incorporated into a model that predicts membership in a BCG tier for a given stream as a function of watershed processes. These processes are affected by the degree of urban development in the watershed, which interacts with stressors such as hydrology and stream chemistry, and affects the aquatic biota found in the streams.

\section{Bayesian Network Model}

Conventional bivariate analysis of stream ecosystem data is often focused on relations between single pairs of environmental factors, without incorporating the influence of additional interconnected factors or previous ecological information about the system. Multiple factors associated with reach-scale hydrology, habitat, and water chemistry processes can act as stressors that affect the biological community, either through direct pathways (solid arrows) or through interactions among the processes (dashed arrows) (fig. 1). A Bayesian network modeling approach (Pearl, 1988) examines the relations among the processes by dissecting this complex system into smaller sets of directly related factors.

The two main components of a Bayesian network are "nodes," representing important concepts (processes and their factors), and "arrows," representing relations between those concepts in the form of conditional probabilities (that is, the likelihood of any particular outcome depends on the occurrence of preceding events). To depict the structure of a model, these nodes and arrows are combined into a graphical representation with the use of a directed acyclic graph (DAG). As shown in figure 5, for example, Urban development can indirectly affect Biological condition by first disrupting Physical condition and Chemical condition. The relations among these four major concepts are depicted with a DAG through a set of nodes connected by ordered arrows where no pathway following the arrows goes through the same node twice; that is, there are no cycles (feedback loops) in the model. A DAG describing this system, therefore, uses four nodes and four arrows.

A node from which an arrow points is called a "parent" node and a node toward which an arrow points is called a "child" node. Therefore, Urban development is the parent of 


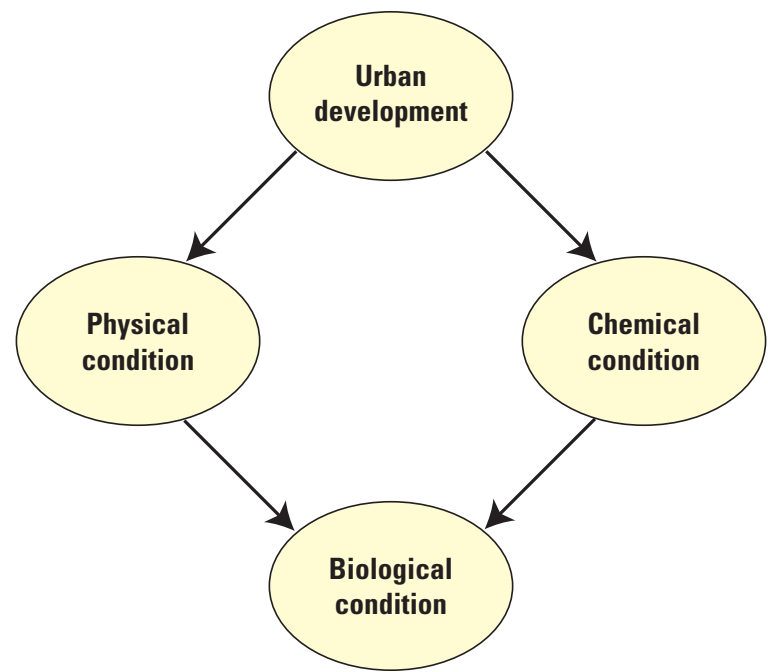

Figure 5. Directed acyclic graph (DAG) showing a simple Bayesian network with four nodes and four arrows.

both Physical condition and Chemical condition. This notation means that the physical condition and chemical condition are both conditional upon a watershed's urban development level. Child nodes relative to one concept can then be parent nodes of another concept. That is, in addition to being children of Urban development, Physical condition and Chemical condition are both also parents of Biological condition.

This modeling structure allows for the deconstruction of a complicated system into sets of direct relations between every parent node and its children, so that child nodes can then be modeled as parents of subsequent child nodes to eventually link the entire system into one probabilistic network. Representing a network of associations as a series of conditional relations such as these reduces the difficulty of parameterizing such a model and also greatly increases the amount of information obtainable from model output. One needs only to specify relations between directly linked factors in order to be able to create a Bayesian network that describes the interrelations among all factors simultaneously. The effect of changing one factor can then be measured on all remaining factors.

To turn this qualitative model structure into a quantified Bayesian network, conditional probability tables (CPT) are defined, either using expert knowledge or existing data. For every possible combination of parent node values, a CPT indicates a probability distribution for values likely to occur at each child node. In a simplified example where urban development can be classified as either low or high, and Urban development is the single parent of Physical condition, the CPT for Physical condition has two rows: low and high urban development (fig. 6). Each row provides the probability of observing either good or poor physical condition given a known level of urban development. The probabilities in each row must add up to 100 percent, because each row describes a complete distribution across all possible child node values. The first row of this CPT, relating Urban development and Physical condition, indicates that if Urban development is low, there is a 90 percent chance that Physical condition is good, and a 10 percent chance that Physical condition is poor. Put differently, if 100 streams were selected from watersheds having low urban land cover, 90 streams are likely to have good physical characteristics, but 10 could have poor characteristics.

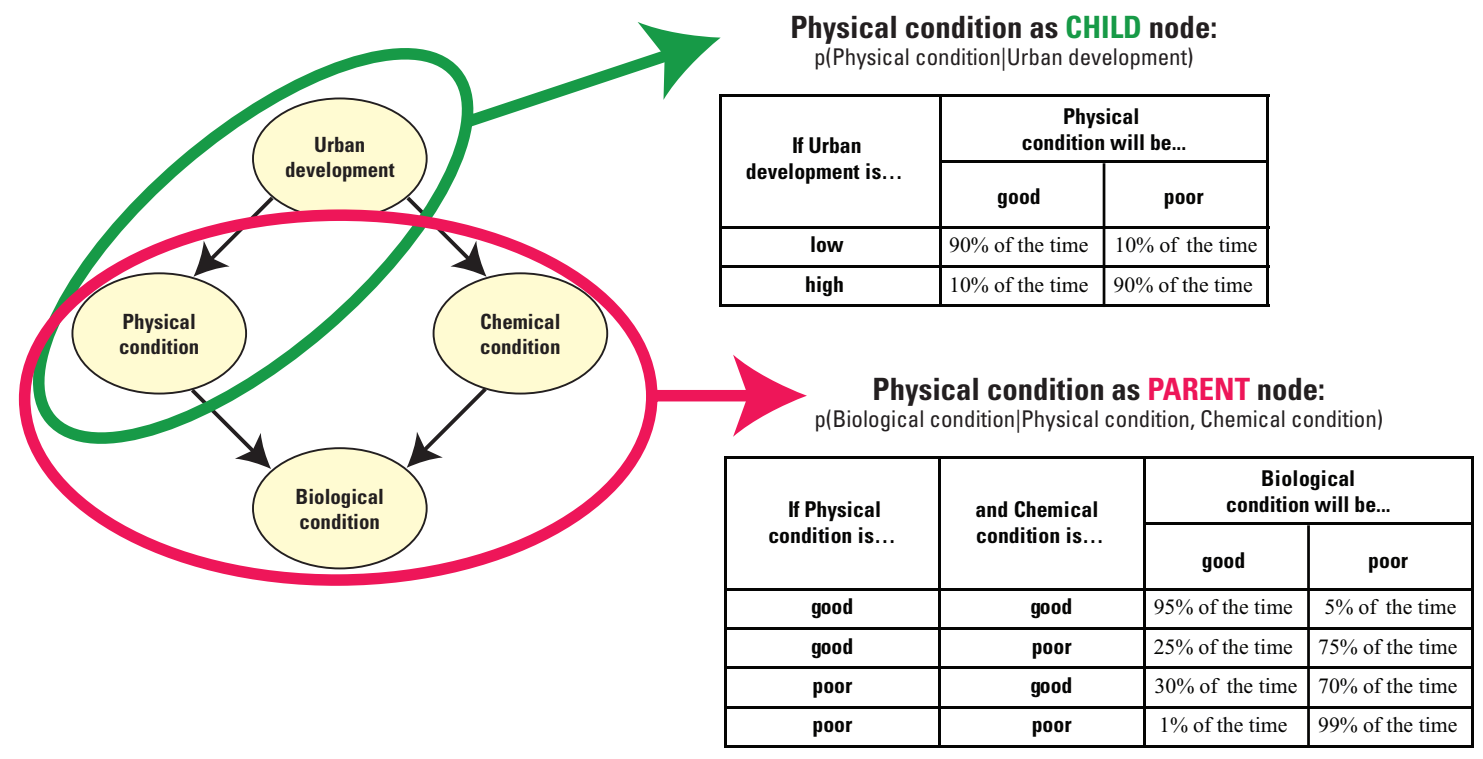

Figure 6. Example of simple conditional probability tables (CPT). The relation between each child node and its parents (left) is specified with a CPT (right). The upper CPT describes the relation when Urban development is the parent node and Physical condition is the child node. The lower CPT describes the relation when Physical condition is the parent node (along with the Chemical condition parent node) and Biological condition is the child node. 
A primary advantage of the Bayesian network over traditional methods of causal analysis is the ability to simultaneously model interacting system components. In the simplified example, Biological condition is affected by both Physical condition and Chemical condition, so the associated CPT shows four probability distributions for Biological condition that account for all possible combinations of physical and chemical stream states (fig. 6, lower table). The first row of the CPT for Biological condition indicates a 95-percent chance that Biological condition is good if Physical condition and Chemical condition are both good. The probability of observing good Biological condition decreases to 30 percent or 25 percent, respectively, if either Physical condition or Chemical condition is poor and decreases to 1 percent if both Physical condition and Chemical condition are poor.

\section{Creating a Bayesian Network Model}

Creating a Bayesian network involves two major steps: (1) developing an initial model and distributions, known as the prior model, because it is built from information known prior to the inclusion of a specific dataset, and (2) updating the prior model with data to obtain the posterior model, which is the model form after data have been incorporated. All aspects of the prior model used for the present study were based on elicitations from subject experts such as scientists and resource managers. The information obtained included the model structure (arrangement of nodes and arrows), variable selection and discretization (division of a continuous measurement into discrete categories), conditional probabilities (the probabilities that child nodes will have certain values conditional upon the states of parent nodes), and prior weights (the influence of prior information on the model, relative to the influence of data). The prior model is then updated with the EUSE Northeast data to create the posterior model. As per Bayesian philosophy (Bayes, 1763), prior knowledge of how a system works is updated when additional information about the system is available. Upon completion, this posterior model can be used to better understand the relations within the parameterized network of nodes through the propagation of "evidence," also called predictive or diagnostic probability calculation (Jensen and Nielsen, 2007). By setting the value of any parent (driver) node, a Bayesian network can predict, in distribution form, the likely values of all child (endpoint) nodes ultimately affected by changes to that driver. A Bayesian network can also "diagnose" the likely distributions of parent nodes required to cause a set child node value. In both cases (either following the arrow from parent to child, or in reverse), using the probabilistic relations between the nodes and Bayesian statistics, the Bayesian network will update what is known about a node on the basis of actual or hypothetical evidence about another node. The Bayesian networks presented in this report were built using Hugin software (Hugin, 2008).

\section{Developing the Northeastern U.S. Bayesian Network Prior Model Using Expert Elicitation}

Expert elicitation specifically refers to the translation of expert knowledge into a probabilistic framework (Morgan and Henrion, 1990; Winkler, 2003; Reckhow and others, 2005; O'Hagan and others, 2006). Scientists often prefer to describe the model development process as objective, without acknowledging that model and parameter selections are made by way of implicit expert judgment. Expert elicitation, in contrast, is an explicit incorporation of expert judgment into the modeling process. The goal is to codify expert knowledge, appropriately recognizing the degree of uncertainty in that knowledge, and then use that knowledge directly in model development and parameterization. Using expert elicitation in model development thus focuses on the science of the problem by confirming or denying expected relations (hypothetico-deductive reasoning), instead of calculating statistics for all possible models and variable combinations without clear purpose.

Expert knowledge is essential to the development of the prior portion of the Bayesian network modeling construct described herein. Unlike data gleaned from a single study, experts provide an integrated estimate of system relations and uncertainties based on all information obtained and synthesized over their respective careers. Weighting prior knowledge in which experts have high confidence more heavily than lowconfidence knowledge can help address known problematic data concerns.

To develop this Northeastern U.S. Bayesian network prior model, multiple groups of experts were recruited that had long-term experience in urban planning, water management, water chemistry, basin-scale habitat evaluation, and aquatic ecology (table 2). Following a specific methodology that was designed for creating an informed expert prior Bayesian network (Kashuba, 2010), model structure, variable selection and discretization, conditional probabilities, and prior weights were elicited from these groups of subject matter experts. 
Table 2. Northeastern U.S. urbanization effects model expert teams.

\section{Biologists and aquatic ecologists}

Susan P. Davies, Maine Department of Environmental Protection

David L. Courtemanch, Maine Department of Environmental Protection

Thomas J. Danielson, Maine Department of Environmental Protection

Susan K. Jackson, U.S. Environmental Protection Agency, Headquarters

Jeroen Gerritsen, Tetra Tech, Inc.

James F. Coles, U.S. Geological Survey, New Hampshire-Vermont Water Science Center

Thomas F. Cuffney, U.S. Geological Survey, North Carolina Water Science Center

Amanda H. Bell, U.S. Geological Survey, Wisconsin Water Science Center

\section{Water management assessors}

Peter K. Weiskel, U.S. Geological Survey, Massachusetts-Rhode Island Water Science Center

Marilee A. Horn, U.S. Geological Survey, New Hampshire-Vermont Water Science Center

Karen M. Beaulieu, U.S. Geological Survey, Connecticut Water Science Center

Marcus C. Waldron, U.S. Geological Survey, Massachusetts-Rhode Island Water Science Center

\section{Habitat scientists}

Faith A. Fitzpatrick, U.S. Geological Survey, Wisconsin Water Science Center

Marie C. Peppler, U.S. Geological Survey, Wisconsin Water Science Center

Barbara C. Scudder, U.S. Geological Survey, Wisconsin Water Science Center

David S. Armstrong, U.S. Geological Survey, Massachusetts-Rhode Island Water Science Center

Urban planners and managers

Paul E. Sturm, Center for Watershed Protection

William Stack, City of Baltimore Department of Public Works, Water Quality Management Service

Kernell G. Ries, U.S. Geological Survey, Maryland Water Science Center

Ronald E. Bowen, Anne Arundal County, Maryland, Department of Public Works

Janis S. Markusic, Anne Arundal County, Maryland, Department of Public Works

Christopher Victoria, Anne Arundal County, Maryland, Department of Public Works

Joseph MacDonald, American Planning Association

Karen Cappiella, Center for Watershed Protection

Hala E. Flores, Anne Arundal County, Maryland, Department of Public Works

\section{Model Structure}

Model structure refers to the arrangement of Bayesian network nodes and arrows. The goal of structure elicitation is to construct a diagram of nodes and arrows that represent the chain of events through which urban development affects aquatic macroinvertebrate communities. Initial model structure was elicited by way of textual analysis of the transcript from an open-ended causal narrative interview with a USGS career ecologist. Using this causal map development method (Nadkarni and Shenoy, 2004), the expert was asked to describe, step by step, how elements of urban development contribute to a biologically degraded stream condition. Then, formal textual analysis identified major concepts and causal relations between concepts. A "concept" is a single idea, such as "urban development" or "habitat." Environmental processes are represented in the causal relations between concepts, for example, channel incision is one way in which urban development affects habitat. To model the environmental processes discussed in the interview, elicited concepts and relations were converted into structure nodes and arrows, respectively. In model structure development, it is important to eliminate reiteration of the same concepts, clearly define causal pathways, avoid scale mismatch between concepts, and balance model complexity with an ability to parameterize (Reckhow, 1999). Therefore, in an iterative process of expert feedback from all subject matter groups, the initial causal map was pared down to a parsimonious Northeast model structure that was affirmed by expert consensus to represent key processes and causal influences at the selected scale, while also being of sufficiently manageable size to allow for parameterization (fig. 7).

Macroinvertebrate metrics were selected using a twopronged approach. After the process of BCG tier assignment (described earlier), experts were asked to identify the three most vital macroinvertebrate metrics whose values influenced assessment of biological condition. Simultaneously, classification and regression tree (CART) and ordered logistic regression (OLR) models were constructed to evaluate which metrics were most predictive of BCG in the USEPA New England Wadeable Streams (NEWS) dataset (Snook and others, 2007). The three top macroinvertebrate metrics identified by both experts and statistical analysis were chosen for use in the Bayesian network model: generic richness, filter feeder relative abundance, and Plecoptera and Ephemeroptera $(\mathrm{P}+\mathrm{E})$ relative abundance (fig. 7). 


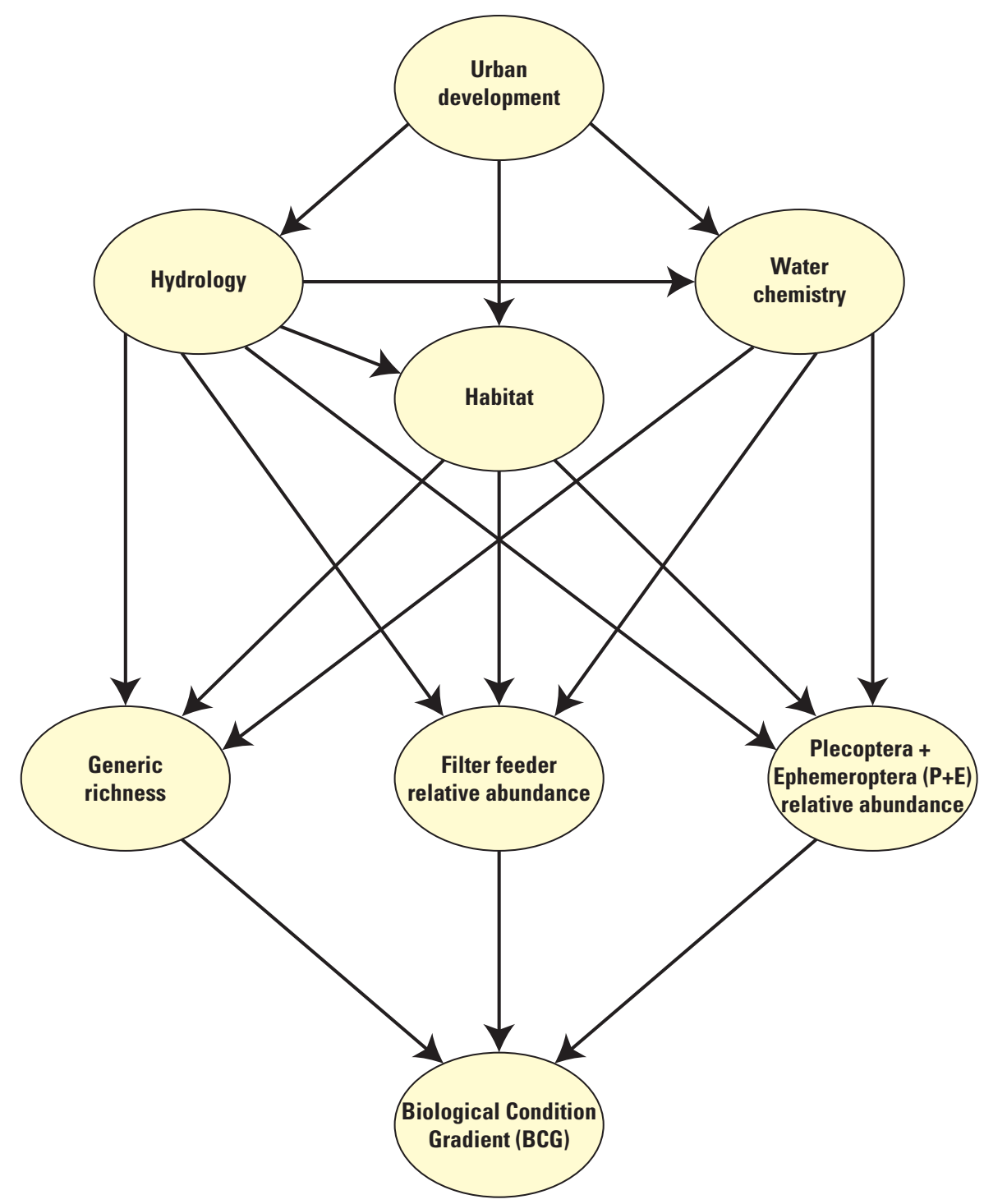

Figure 7. Parsimonious Northeast Bayesian network model structure.

\section{Variable Selection and Discretization}

Model structure development involves only identifying major qualitative concepts (nodes) and their causal connections with each other (arrows). To transform such a causal map into a Bayesian network, a quantifiable variable must be assigned to each node, and each assigned continuous variable must be converted into a discrete number of mutually exclusive categories. For each model node, experts rely on career experience, inter-expert discussion, and consensus building to select an EUSE Northeast dataset variable that best represents the intended concept of that node (table 3). The choice of how many categories a variable should be split into involves a tradeoff between the model's discriminatory strength and the accuracy of (potentially complex) parameterization. Having few categories limits the ability of the model to differentiate between different states of a node and to evaluate changes to the variable and the system. As a variable is split into more categories, however, it becomes more difficult to establish dependencies between model variables (Uusitalo, 2007) because parameter space expands rapidly. In addition, based on lessons learned during method development (Kashuba, 2010), experts often have difficulty distinguishing between the middle two of four categories; therefore, each continuous variable was split into three categories. For the two selected discrete variables (dominant substrate and BCG), model categories were chosen to mimic natural, discrete categories; dominant substrate is binary (either fine or coarse), and BCG has six tiers (table 3 ).

Ideally, each category should represent a group of values similar to one another and distinct from other categories, because after discretization, every value within a single category will be treated identically by the model. In the absence of clear ecological or resource management dividing lines (which dominant substrate and BCG have), this 
Table 3. Nodes and variables chosen by experts to represent major system components in Northeastern U.S. Bayesian network model.

[\%, percent, >, greater than, $\mathrm{P}+\mathrm{E}$, Plecoptera and Ephemeroptera; BCG, Biological Condition Gradient]

\begin{tabular}{|c|c|c|c|c|}
\hline \multirow{2}{*}{ Node } & \multirow{2}{*}{ Variable: units } & \multicolumn{3}{|c|}{ Discrete categories } \\
\hline & & Low & Medium & High \\
\hline Urban development & $\begin{array}{l}\text { Urban land cover: percentage urban land } \\
\text { cover in basin area }\end{array}$ & $0-7 \%$ & $>7-31 \%$ & $>31-100 \%$ \\
\hline Hydrology & $\begin{array}{l}\text { Flashiness: rises greater than } 7 \text { times the } \\
\text { annual median rise }\end{array}$ & 0 & $1-3$ & $4+$ \\
\hline Habitat & $\begin{array}{l}\text { Substrate: dominant ( }>50 \% \text { of transects) } \\
\text { substrate type }\end{array}$ & $\begin{array}{l}\text { Fine } \\
\text { (sand and smaller) }\end{array}$ & & $\begin{array}{c}\text { Coarse } \\
\text { (gravel and larger) }\end{array}$ \\
\hline Water quality & $\begin{array}{l}\text { Specific conductance: at low base flow, } \\
\text { microsiemens per centimeter at } 25 \text { degrees } \\
\text { Celsius }\end{array}$ & $0-139$ & $>139-269$ & $>269$ \\
\hline Generic richness & $\begin{array}{l}\text { Generic richness: total number of taxa at the } \\
\text { genus taxonomic level }\end{array}$ & $0-14$ & $15-37$ & $38+$ \\
\hline BCG & $\begin{array}{l}\text { Biological Condition Gradient discrete scale } \\
\text { of } 1 \text { (best) to } 6 \text { (worst) }\end{array}$ & 1 & 3 & 5 \\
\hline
\end{tabular}

unambiguous distinction between categories is difficult to achieve. Therefore, because no clear thresholds existed for the seven continuous variables requiring discretization, an adaptive, two-criterion category-definition methodology was created. This approach starts with defining category endpoints such that each category contains an equal frequency of available data. These initial category endpoints are then adjusted as ecologically important cutoff points are identified during conditional probability elicitation. Applying this methodology, the 30 EUSE Northeast data points for each continuous variable were split such that values less than the 33rd percentile defined the "low" category, values between the 33rd and 67 th percentiles defined the "medium" category, and values greater than the 67th percentile defined the "high" category. Experts were asked whether these three category definitions accurately represented what would be considered ecologically "low," "medium," or "high" for each variable, and initial endpoint adjustments were made based on expert feedback. During conditional probability elicitation, experts refined their definitions of each category in the context of describing the behavior of variables in relation to other variables. For example, the definition of low generic richness was narrowed from 0-23 genera to 0-14 genera when it was determined that the original range of values was too broad to have a consistent effect on BCG tier classification. All generic richness values defined as low based on frequency did not belong to the same category ecologically, so the category definition was adjusted.

\section{Conditional Probability Tables}

Just as variable selection and discretization explicitly define model nodes, CPTs explicitly define the relations between the nodes (which are represented by arrows). In a Bayesian network, a node is modeled in terms of the probabilities that a sample (in this case, a stream measurement) would belong to each of its categories. This probability distribution across the states of a given node changes depending on the states of nodes that influence it. Rephrasing in Bayesian network parlance, child nodes (into which arrows point) are conditionally dependent on their parent nodes (from which arrows point). For example, the likelihood of observing high, medium, or low flashiness in a stream is conditional on the level of urban development in the stream's watershed. Therefore, the Hydrology child node is parameterized conditional on its Urban development parent node. The Bayesian network structure specifies, through its arrow placement, the set of CPTs required by the model, where a CPT is created for every child node conditional on its parents.

Within each CPT, a probability distribution across all possible states of a child node must be elicited for every possible combination of parent node states. For example, the CPT for the Hydrology node conditional on the Urban development node has a separate distribution across high, medium, and low flashiness for each of the three possible states of urban land-cover percentage (table 4A). Each of these distributions across child node states is represented in its own CPT row and 
Table 4. "Hydrology" node prior conditional probability table and prior weight $\left(\alpha_{0}\right)$, data table, and posterior conditional probability table and posterior weights $\left(\alpha_{0}+n\right)$.

\section{A. Prior conditional probability table}

[Flashiness categories in probability units and $\alpha_{0}$ in equivalent data points; rows selected for prior weight elicitation are highlighted in gray]

\begin{tabular}{ccccc}
\hline \multirow{2}{*}{$\begin{array}{c}\text { Urban land } \\
\text { cover }\end{array}$} & Low & Medium & High & $\boldsymbol{\alpha}_{0}$ \\
\cline { 2 - 5 } & 0.20 & 0.70 & 0.10 & 9.72 \\
\hline Low & 0.15 & 0.55 & 0.30 & 9.72 \\
Medium & 0.10 & 0.40 & 0.50 & 9.72 \\
\hline High & & & & \\
\hline
\end{tabular}

\section{B. Data table}

[Flashiness counts and $n$ total number of samples per parent state combination]

\begin{tabular}{ccccc}
\hline \multirow{2}{*}{$\begin{array}{c}\text { Urban land } \\
\text { cover }\end{array}$} & \multicolumn{4}{c}{ Flashiness greater than $\mathbf{7}$ times the median } \\
\cline { 2 - 5 } & Low & Medium & High & $\boldsymbol{n}$ \\
\hline Low & 1 & 8 & 1 & 10 \\
Medium & 1 & 5 & 4 & 10 \\
High & 2 & 4 & 4 & 10 \\
\hline
\end{tabular}

\section{Posterior conditional probability table}

[Flashiness categories in probability units and $\alpha_{0}+n$ in equivalent data points]

\begin{tabular}{ccccc}
\hline \multirow{2}{*}{$\begin{array}{c}\text { Urban land } \\
\text { cover }\end{array}$} & \multicolumn{4}{c}{ Flashiness greater than $\mathbf{7}$ times the median } \\
\cline { 2 - 5 } & Low & Medium & High & $\boldsymbol{\alpha}_{0}+\boldsymbol{n}$ \\
\hline Low & 0.149 & 0.751 & 0.100 & 19.72 \\
Medium & 0.125 & 0.525 & 0.351 & 19.72 \\
High & 0.151 & 0.400 & 0.449 & 19.72 \\
\hline
\end{tabular}

described by its own Dirichlet prior distribution (Jensen and Nielsen, 2007) (app. 1, eqs. 1.1, 1.2). A Dirichlet distribution describes the probabilities of each of three or more mutually exclusive events occurring.

To fully characterize the seven CPTs defined by this 8-node, 17-arrow Bayesian network model (fig. 7), 102 such Dirichlet distributions are required, collectively composed of a total of 378 Dirichlet $\theta_{i}$ parameters (where $i=1,2$, or 3 for all nodes except Habitat, which has two states, or $B C G$, which has six). Nodes with no parents, called "marginal nodes," (such as Urban development) are simply assigned an unbiased flat distribution across all possible discrete states, such that every state has an equal probability of occurrence. Dirichlet distributions for the urban development node are never explicitly reported because urban land cover is simply distributed equally ( 0.33 per node state), by design, for both the prior distribution and the data distribution and, hence, the posterior distribution. The roles of prior, data, and posterior are described in further detail later as part of the discussion on updating the prior model with data.

The CPTs should be elicited following a systematic, prescribed process designed to guide the experts through a rational, judicious evaluation of their expertise. There are several documented direct and indirect probability elicitation methods for binomial distributions (Winkler, 1967, 2003; Morgan and Henrion, 1990); however, the literature remains sparse on ideal elicitation methods for the multinomial distributions used in this model. Therefore, an adaptation of the direct fixed value elicitation method (Clemen, 1991) was developed to apply to multinomial elicitations, following the five steps of the Stanford Research Institute Elicitation Protocol (Spetzler and von Holstein, 1975) to attempt to minimize bias and error. To start this elicitation, experts are familiarized with the problem context (a process called "motivating") and specific questions of interest ("structuring") and are then asked to summarize and review relevant expert knowledge qualitatively ("conditioning"). After experts are appropriately prepared, prior 
probabilities are assigned ("encoding") and tested to ensure they correctly capture the experts" opinions ("verifying").

Encoding was conducted in frequency units rather than probability units to facilitate human conceptualization of the information required (Morgan and Henrion, 1990). Using direct fixed value multinomial-adapted elicitation, experts were asked to divide a fixed total of events into categories according to their expected likelihood of occurrence. For example, for the first row of the Hydrology child node conditional on the Urban development parent node, the experts are asked the following:

Assume the watersheds of 100 streams in the Northeastern United States are known to have 0-7 percent (low) urban land cover. Assume all other stream features are randomly distributed as if you took a random sample of northeastern streams with low urban land cover. How many of those 100 streams would you expect to have low flashiness (no rises greater than 7 times the median in a year), medium flashiness (between 1 and 3 rises), or high flashiness (more than 4 rises)?

Experts were encouraged to discuss this question until consensus was reached. In this example, experts agreed to report that, out of 100 streams in watersheds with low urban land cover, they would expect 20 of those streams to have low flashiness, 70 streams to have medium flashiness, and 10 streams to have high flashiness (table $4 A$, first row). This same exercise was then conducted for the remaining possible Urban development parent node states (that is, medium and high) and then also for each CPT row (that is, parent node state combination) of the remaining six child nodes. For the three macroinvertebrate metric nodes and $B C G$ node, a total of 1,000 streams were postulated to allow for the assignment of very unlikely probabilities of less than 1 percent. Frequency results were converted into probabilities and normalized when the reported total did not equal 100 or 1,000 exactly. The complete elicited prior CPT for child node Hydrology is reported in table $4 A$. All other prior CPTs are provided in appendix 2, tables $2-1 A$ to $2-6 A$.

\section{Prior Weights}

Prior weights were elicited to determine how much to weight prior knowledge relative to data during Bayesian updating (described later). Prior weights are reported in units of "equivalent data points" to allow for comparison with data weight reported as a number of actual data points. After evaluation of three different prior weight-elicitation methods, the probability range method (Cowell and others, 1999) was found to show lowest within-method variability, most consistent prior weights among nodes, and was most intuitive for experts to communicate (Kashuba, 2010). This method asks experts to provide a range of values that represents their certainty about a given parameter. For example, experts reported in CPT elicitation that, of 100 streams having low urban land cover, they would expect 20 to have low flashiness. When asked to provide a certainty range of values, they responded that they would not be surprised if there were as few as 5 or as many as 35 streams that had low flashiness in a sample of 100 low urban land-cover streams (table 5, first row). The premise of the probability range elicitation method is that small reported probability ranges correspond to high certainty (that is, high prior weight), and large ranges correspond to high uncertainty (low prior weight).

The width of an elicited range has been determined to be approximately two standard deviations long around a Dirichlet $\theta_{i}$ value (app.1, eq. 1.1) (Spiegelhalter and others, 1994), that is, one standard deviation is equivalent to one-half the length of the range. Eliciting a prior probability and an uncertainty range of probabilities for a CPT cell, therefore, provides values for expected mean $\left(E\left[\theta_{i}\right]\right)$ and expected variance $\left(\operatorname{var}\left[\theta_{i}\right]\right)$, respectively, for the Dirichlet parameter, $\theta_{i}$. Equations for the Dirichlet expected value and Dirichlet expected variance can then be solved for prior weight, $\alpha_{0}$, (app. 1, eqs. 1.3-1.5) (Kashuba, 2010). In the example reported earlier, $E\left[\theta_{i}\right]=0.20$, a range of 0.30 translates to a standard deviation of 0.15 , so $\operatorname{var}\left[\theta_{i}\right]=0.0225$, resulting in $\alpha_{0}=6.11$ for the CPT cell representing the probability of observing low flashiness given low urban land cover (table 5, first row). Probability ranges were elicited and prior weights were calculated in this way for two random rows of each child node's CPT (highlighted in table $4 A$ for Hydrology child node, and app. 2 , tables $2-1 A$ to $2-6 A$ for remaining child nodes). These celllevel prior weight values were then averaged across a child node for stability (table 5). Node-averaged prior weights are also reported with elicited CPTs in table $4 A$ and appendix 2, tables $2-1 A$ to $2-6 A$. For example, the prior weight for the Hydrology child node, 9.72 (table $4 A$, table 5), is the mean of the six elicited probability ranges for the low and high urban land cover CPT rows (table 5, first six rows). 
Table 5. Probability range prior weight elicitation results for all child state probabilities in each of two randomly selected rows from each conditional probability table.

$[\mathrm{P}+\mathrm{E}$, Plecoptera and Ephemeroptera; BCG, Biological Condiiton Gradient]

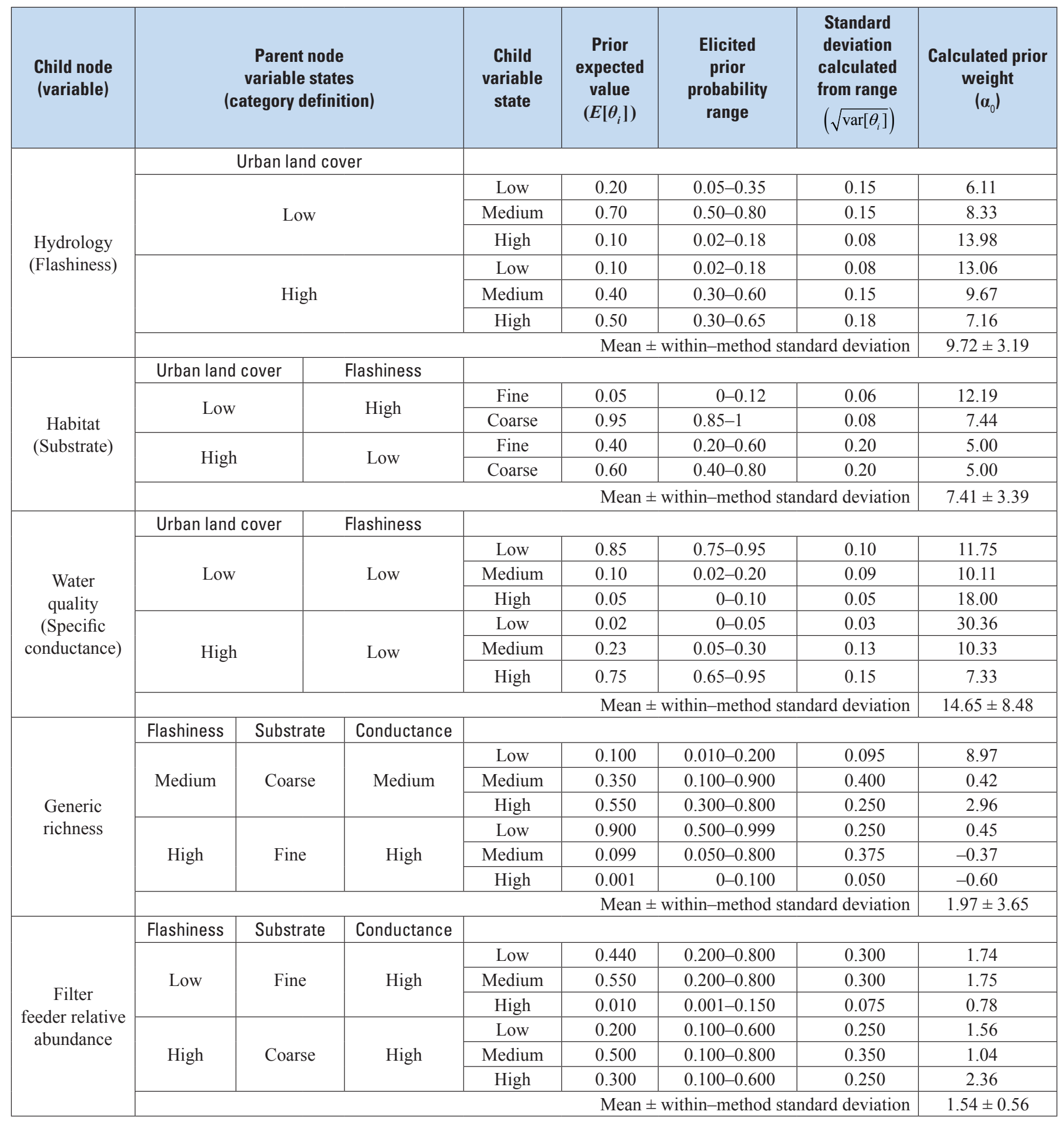


Table 5. Probability range prior weight elicitation results for all child state probabilities in each of two randomly selected rows from each conditional probability table.-Continued

[P+E, Plecoptera and Ephemeroptera; BCG, Biological Condiiton Gradient]

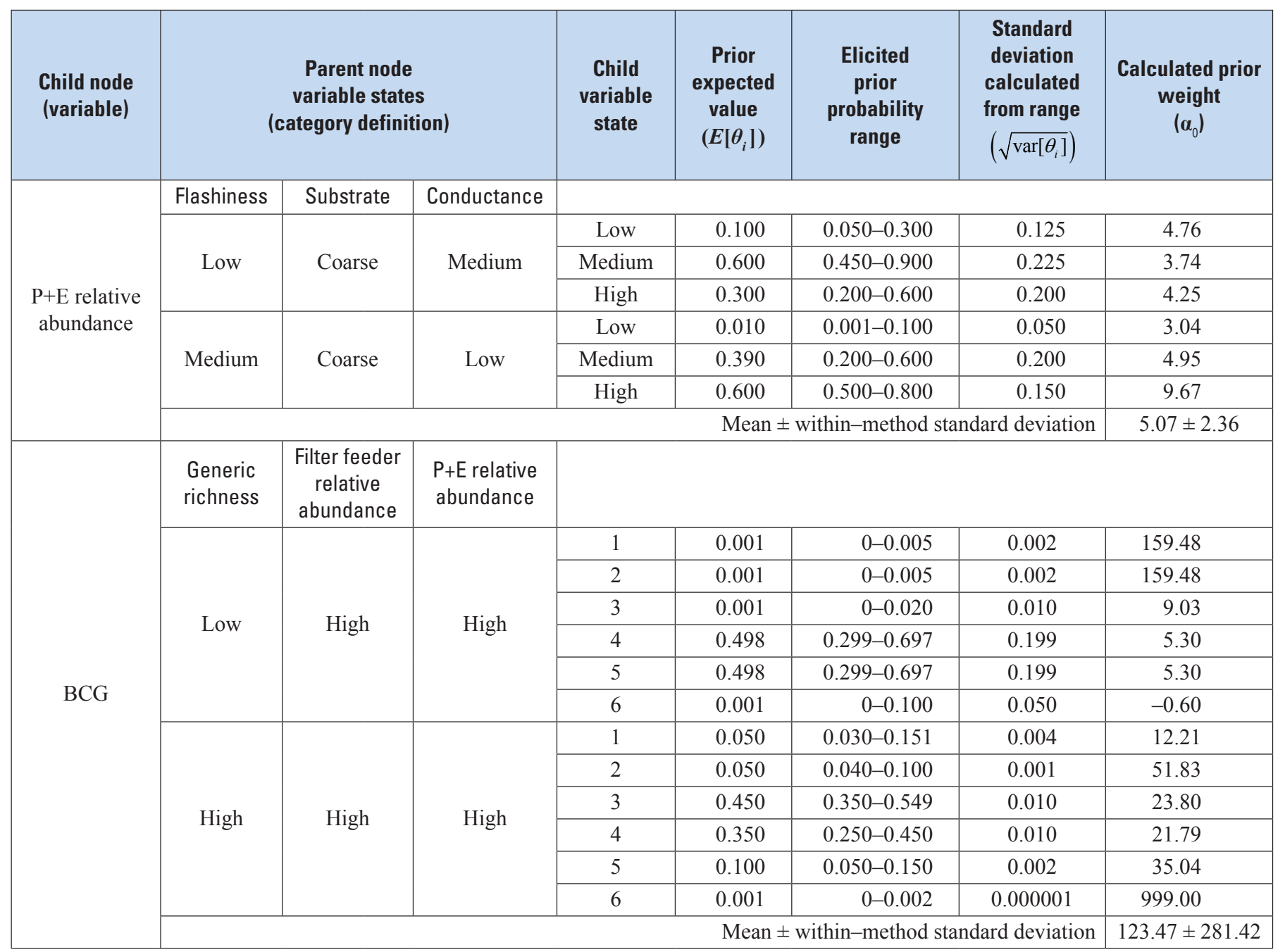

\section{Updating the Prior Model with Data}

The mechanism of Bayesian network updating and, hence, the ability to combine expert judgment with data derives from the principles of Bayes Theorem (Bayes, 1763):

$$
p(\theta \mid x) \propto p(\theta) p(x \mid \theta)
$$

where

$$
\begin{aligned}
& p(\theta) \quad \text { is the prior probability distribution of } \theta \text {, the } \\
& \text { parameter set of interest, }
\end{aligned}
$$

$p(x \mid \theta) \quad$ is the likelihood function characterizing $x$, the data, given $\theta$, and

$p(\theta \mid x) \quad$ is the posterior probability distribution of $\theta$, given the data, $x$, which is a combination of the prior information and the data collected.
In general, Bayesian analysis attempts to gain understanding about the distribution of all possible values of a parameter set of interest, $\theta$. This distribution, $p(\theta)$, is originally constructed from information available prior to the collection of a specific dataset. Once data are available, the likelihood function, $p(x \mid \theta)$, calculates the likelihood of observing those data given each possible value of $\theta$. To update the prior information with the additional information from the data, the prior distribution is multiplied by the data likelihood function, resulting in a posterior distribution of $\theta$, conditional upon the data observed. This posterior distribution, $p(\theta \mid x)$, is essentially a weighted average of the prior information and the data (sidebar 2). 


\section{Sidebar 2. How Bayesian Updating Works}

\section{Simple Example with Single Probability Parameter}

Bayesian updating is the process of revising a prior distribution of one or more parameters using newly obtained data that contain information about those parameters. This simple example shows how to estimate the probability that a random stream in the northeastern United States has good biological condition. This probability can be represented by the parameter, $\theta_{i}$. Prior information about $\theta_{i}$ (from either expert knowledge or previous data) can be summarized in this case by a beta distribution centered on 0.50 with a standard deviation of 0.2 (fig. 8, solid red distribution). This prior information is known with a prior weight (hypothetical sample size) of 6 . This means that, prior to data collection, analysts believe there is a 50 -percent chance that a random stream in the northeastern United States has good biological condition, and the certainty of that probability estimate is equivalent to the certainty of observing six streams, of which three had good biological condition. This prior distribution summarizes everything known, and how well it was known, before data were collected.

The biological condition of 10 random streams in the Northeast are then sampled. The data collected indicate that 7 of these 10 streams have good biological condition. This means the data are assumed to belong to a binomial likelihood distribution centered on the maximum likelihood estimate of 0.70 for parameter $\theta_{i}$ (fig. 8, dashed blue distribution). Making an inference about parameter $\theta_{i}$ based on these data alone, there is a 70-percent chance that a random stream in the northeastern United States has good biological condition with a certainty equivalent to 10 samples.

Given the sources just described, there are two different types of information from which two different conclusions can be drawn concerning the probability that a stream in the Northeast United States has good biological condition. There is slightly more certainty in the conclusions drawn from the 10 sample units of recently collected data, but that does not mean that the 6 sample units of information available prior to collecting data should be discounted.

Ideally, the greatest certainty would be associated with a conclusion that could be drawn from both aforementioned sources of information. This combining of information is exactly what Bayesian updating allows one to do. By taking a weighted average of prior information and data, a posterior (beta) distribution for parameter $\theta_{i}$ is calculated with an expected value of 0.625 (fig. 8, solid purple distribution). An analyst can now infer that the probability of a stream in the northeastern United States having good biological condition is closer to 62.5 percent, with a certainty equivalent to 16 samples. This greater certainty of the posterior state of knowledge is represented by a smaller standard deviation of 0.1 (fig. 8, solid purple distribution) relative to the prior distribution standard deviation of 0.2 (fig. 8, solid red distribution). 


\section{Bayesian Updating for Multiple Probability Parameters within a Bayesian Network CPT Row}

In the context of a Bayesian network, Bayesian updating of parameters applies to each parameter in each conditional probability table (CPT). Rather than using the distribution forms that describe the probability of a single outcome occurring (that is, beta prior and posterior distributions for the parameter, $\theta$, and binomial likelihood for the data), each row in the conditional probability table uses the distribution forms that describe the probability of one of multiple outcomes occurring. The distribution form for multiple (for example, three) possible outcomes is Dirichlet prior and posterior distributions for the parameters, $\theta_{1}, \theta_{2}, \theta_{3}$, and multinomial likelihood for the data, as described in the discussion of model updating with prior data and equations 1.1-1.9 (appendix 1).

The different distribution forms just described follow identical Bayesian principles. Each cell in a CPT shows the expected value of the probability that a stream will fall into that child node category given that it belongs to the parent node category for that row. For example, the first row of the prior CPT for the Hydrology child node shows that for a stream in a watershed with low urban development (parent node category), there is an expected 20-percent chance that stream will have low flashiness, a 70-percent chance that stream will have medium flashiness, and a 10-percent chance that stream will have high flashiness (fig. 9, top prior row, from table $4 A$, first row). Similar to the single probability example described earlier, these three probabilities are constructed from (expert) knowledge obtained prior to data collection. These three values are considered the expected prior values for Dirichlet parameters, $\theta_{1}, \theta_{2}, \theta_{3}$. The expert-elicited prior weight indicates that this knowledge is equivalent in certainty to 8.3 hypothetical samples. This prior weight is represented in Dirichlet terminology as $\alpha_{0}$ (appendix 1, equations 1.1-1.9).

Additionally, Effects of Urbanization on Stream Ecosystems (EUSE) Northeast data measuring flashiness and urban land cover are also available to make inference on the likely values of $\theta_{1}, \theta_{2}, \theta_{3}$. In this case, data show that of 10 streams in watersheds having low urban land cover, 1 has low flashiness, 8 have medium flashiness, and 1 has high flashiness (fig. 9 , middle data row, from table $4 B$, first row). The maximum likelihood values for $\theta_{1}, \theta_{2}$, and $\theta_{3}$, given these data, (from assumptions specific to a multinomial distribution) are $0.10,0.80$, and 0.10 , respectively. Bayesian updating is then used to combine these two sets of information into a posterior conditional probability table that lists the expected values for parameters $\theta_{1}, \theta_{2}$, and $\theta_{3}$, given both prior information and data, weighting by the prior weight, $\alpha_{0}$, and sample size, $n$, respectively (fig. 9 , bottom posterior row, from table $4 C$, first row). In this case, because the sample size is slightly greater than the prior weight, posterior expected values of the probabilities of having low or medium flashiness given low urban land cover are calculated slightly closer to the maximum likelihood values of the data compared to the expected values of the prior distribution. The posterior probability

PRIOR
\begin{tabular}{|c|c|c|c|}
\hline $\begin{array}{c}\text { Urban development: } \\
\text { Percent urban land cover }\end{array}$ & \multicolumn{3}{|c|}{$\begin{array}{c}\text { Hydrology: } \\
\text { Flashiness greater than 7 times the annual median rise }\end{array}$} \\
\hline $\begin{array}{c}\text { Low } \\
(\mathbf{0}-\mathbf{7 \%})\end{array}$ & $\begin{array}{c}\text { Low } \\
(0 \text { rises })\end{array}$ & $\begin{array}{c}\text { Medium } \\
(1-3 \text { rises })\end{array}$ & $\begin{array}{c}\text { High } \\
(4+\text { rises })\end{array}$ \\
\hline & $\mathbf{0 . 2 0}$ & $\mathbf{0 . 7 0}$ & $\mathbf{0 . 1 0}$ \\
\hline & $E\left[\theta_{1}\right]$ & $E\left[\theta_{2}\right]$ & $E\left[\theta_{3}\right]$
\end{tabular}

DATA

\begin{tabular}{|c|c|c|c|}
\hline $\begin{array}{c}\text { Urban development: } \\
\text { Percent urban land cover }\end{array}$ & \multicolumn{3}{|c|}{$\begin{array}{c}\text { Hydrology: } \\
\text { Flashiness greater than 7 times the annual median rise }\end{array}$} \\
\hline $\begin{array}{c}\text { Low } \\
(\mathbf{0 - 7 \% )}\end{array}$ & $\begin{array}{c}\text { Low } \\
(0 \text { rises })\end{array}$ & $\begin{array}{c}\text { Medium } \\
(1-3 \text { rises })\end{array}$ & $\begin{array}{c}\text { High } \\
(4+\text { rises })\end{array}$ \\
\hline & $\mathbf{1}$ & $\mathbf{8}$ & $\mathbf{1}$ \\
\hline & $x_{1}$ & $x_{2}$ & $x_{3}$ \\
\hline
\end{tabular}

POSTERIOR

\begin{tabular}{|c|c|c|c|c|}
\hline $\begin{array}{l}\text { Urban development: } \\
\text { Percent urban land cover }\end{array}$ & \multicolumn{3}{|c|}{$\begin{array}{l}\text { Hydrology: } \\
\text { Flashiness greater than } 7 \text { times the annual median rise }\end{array}$} & \multirow{3}{*}{$\begin{array}{c}\text { Posterior } \\
\text { weight } \\
18.3\end{array}$} \\
\hline \multirow[t]{3}{*}{$\begin{array}{c}\text { Low } \\
(0-7 \%)\end{array}$} & $\begin{array}{l}\text { Low } \\
(0 \text { rises })\end{array}$ & $\begin{array}{l}\text { Medium } \\
(1-3 \text { rises })\end{array}$ & $\begin{array}{c}\text { High } \\
\text { (4+ rises) }\end{array}$ & \\
\hline & 0.15 & 0.75 & 0.10 & \\
\hline & $E\left[\theta_{1} \mid x_{1}\right]$ & $E\left[\theta_{2} \mid x_{2}\right]$ & $E\left[\theta_{3} \mid x_{3}\right]$ & $\alpha_{0}+n$ \\
\hline
\end{tabular}
of high flashiness remains unchanged because both the prior distribution and the data likelihood have the same $0.10 \theta_{3}$ value. This Bayesian updating process is followed for every CPT row in the model.

8.3

$\alpha_{0}$

Figure 9. Bayesian updating of parameters $\theta_{1}, \theta_{2}, \theta_{3}$, within a conditional probability table (CPT) row in a Bayesian network. The posterior expected values, $E\left[\theta_{1} \mid x_{1}\right]$, $E\left[\theta_{2} \mid x_{2}\right]$, and $E\left[\theta_{3} \mid x_{3}\right]$, are each the weighted average of the prior expected values $\left(E\left[\theta_{1}\right], E\left[\theta_{2}\right]\right.$, and $\left.E\left[\theta_{3}\right]\right)$ and the maximum likelihood estimates from the data $\left(x_{1} / n, x_{2} / n\right.$, and $\left.x_{3} / n\right)$, weighted by the prior weight $\left(\alpha_{0}\right)$ and sample size $(n)$, respectively. 
In theory, prior parameter distributions can take any functional form conceivable. However, if the posterior distribution function cannot be calculated analytically (for a given combination of prior distribution and data likelihood function), numerical approximation techniques such as Markov Chain Monte Carlo (MCMC) sampling are often computationally laborious and time intensive (Lunn and others, 2000). If, instead, the prior distribution and the data likelihood function belong to what is called a conjugate family, then a posterior solution of the same functional form as the prior distribution is guaranteed.

In the context of a Bayesian network, the parameter sets of interest are the probabilities that a node's value will fall within each of its possible discrete categories. These probabilities, represented by different $\theta_{i}$ parameters, can be modeled using a Dirichlet distribution, as explained earlier in the discussion of prior model development using expert elicitation. For example, a node representing urban development has three possible categories: high (>31-100 percent urban land cover), medium ( $>7-31$ percent) and low (0-7 percent). The prior probability that a random Northeastern U.S. watershed has high, medium, or low urban land cover can be described by parameters $\theta_{1}, \theta_{2}$, and $\theta_{3}$, respectively.

Continuous data collected by measuring the urban landcover percentages of a random sample of watersheds can be converted into discrete data by placing the samples into the three defined urban land-cover categories and reporting number of samples in each category rather than each sample's original continuous value. In this way, 30 continuous $x$ values (for example, $0.9,1.1,1.8,2.4,2.4,2.6,3.2,3.3,4.9,6.4,8.0,8.0$, $10.3,15.4,18.3,21.5,23.5,24.4,28.5,30.6,34.7,35.7,38.6$, $43.3,44.7,50.2,53.7,60.1,64.4$, and 76.4 percent urban land cover) can be reported, instead, as three discrete $x$ values (for example, 10,10, and 10 samples in categories high, medium, and low urban development, respectively). Data in this discrete form can be described using the multinomial likelihood function (app. 1, eqs. 1.6-1.7). The Dirichlet-multinomial family is conjugate, which means that if the Dirichlet distribution is used to quantify prior knowledge about the possible values of $\theta_{1}, \theta_{2}$, and $\theta_{3}$, and multinomial data are collected, then the posterior distribution combining prior knowledge and data will also be of the Dirichlet form. To calculate this posterior distribution, the Dirichlet prior distribution is multiplied by the multinomial likelihood function as specified by Bayes Theorem (eq. 1), resulting in a Dirichlet posterior distribution with new coefficient values (app. 1, eqs. 1.8-1.9).

The Dirichlet posterior estimates are a weighted average of prior estimates and data estimates. Hypothetically, assume the expected value of $\theta_{1}$ from the Dirichlet prior distribution was 0.20 with a prior weight of 5 and data showed that 4 out of 10 samples fell into category 1 . The posterior expected value of $\theta_{1}$ calculated from the Dirichlet posterior distribution would then be the average of 0.20 weighted by 5 and 0.40 weighted by 10 , or 0.33 . This posterior expected value of $\theta_{1}$, 0.33 , is the average of 0.20 and 0.40 , where 0.40 has twice the weight of 0.20 , as specified by the prior weight relative to the data weight (sample size). In this way, prior and data information are combined, appropriately weighted (sidebar 2 ). In other words, the prior expected value of $\theta_{1}(0.20)$ is updated with information from collected data to yield a posterior expected value of 0.33 . Specific to the Hydrology child node discussed above, the expert elicited prior CPT shows that a watershed with low urban land cover, flashiness is 20 percent likely to be low, 70 percent likely to be medium, and 10 percent likely to be high (table $4 A$ ). The EUSE Northeast data show that, of 10 watersheds with low urban land cover, 1 had low flashiness, 8 had medium flashiness, and 1 had high flashiness (table $4 B$ ). Therefore, the posterior CPT for the Hydrology child node shows expected values of 14.9 percent low, 75.1 percent medium, and 10.0 percent high flashiness, which are each an average of the prior probabilities and the data, weighted by the prior weight and sample size, respectively (table 4C).

\section{Predicting Effects of Urbanization on Biota}

Once a prior Bayesian network is built and updated with data, the resulting posterior Bayesian network is used to investigate potential results of applying management practices on biological condition. Drawbacks of using only data to parameterize this Bayesian network are presented.

\section{Prior Bayesian Network Model}

The final prior Bayesian network combines elicited model structure, variables, CPTs, and prior weights into a single representation of node relations and uncertainties (app. 2, fig. 2-1). The model shows marginal probability distributions for each of the eight nodes; marginal probabilities are unconditional probabilities for each variable that are calculated by integrating out information about its parent variables. Conditional probabilities and prior weight information are embedded in the arrows pointing from parent to child nodes. For each node in a parameterized Bayesian network like figure 2-1 (app. 2), the horizontal blue bars depict the probability of a Northeast stream sample falling within each node state, with longer bars corresponding to higher probabilities. The numeric column superimposed on these bars lists these probability values as percentages. This prior network can provide information such as the probability of observing a "minimally changed" BCG Tier 2 condition in a random Northeastern U.S. stream is 13.47 percent. More usefully than making an average inference for a random stream, hypothetical "evidence" can be entered into the network (shown with horizontal red bars) and propagated to predict the effects of one variable state change on the rest of the system. For example, under conditions of low urban land cover, the predictive probability of observing BCG Tier 2 condition increases to 22.78 percent (fig. 10). 


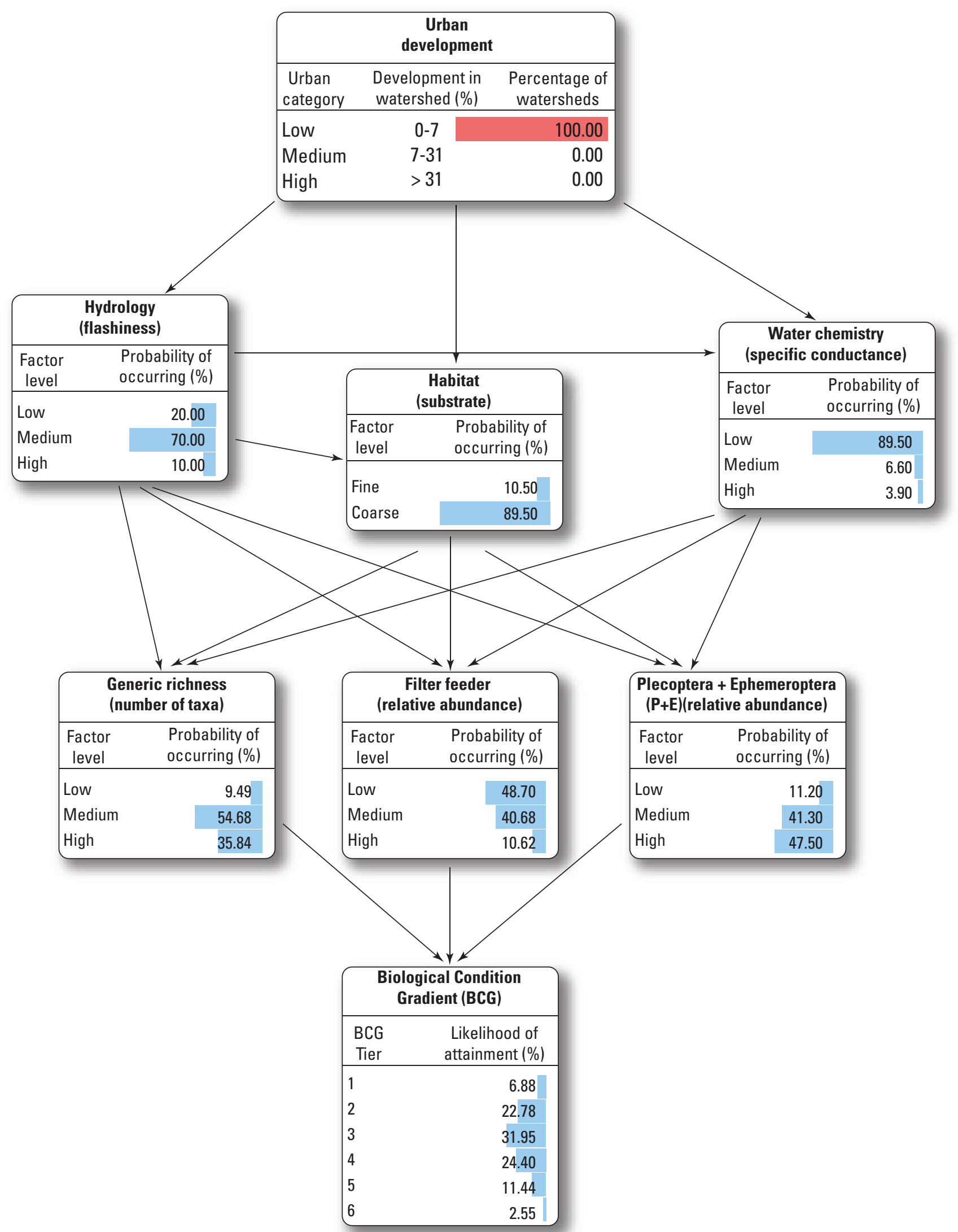

Figure 10. Prior predictive probabilities of occurrence for each level of each node under low urban development. [Probabilities are represented as percentages, numerically and graphically (horizontal colored bars). Factor levels are defined in table 3.] 
In addition, when urban land cover is known to be low, the prior predictive probabilities indicate an increased likelihood of observing stable hydrology, coarse habitat, lowconductance water quality, high macroinvertebrate generic richness, low filter feeder relative abundance, and high $\mathrm{P}+\mathrm{E}$ relative abundance. These predictions are reversed under conditions of high urban land cover, with the probability of observing BCG Tier 2 condition decreasing to 5.69 percent (fig. 11). Not only does the Bayesian network illustrate the direction and magnitude of expected change for every node, it also incorporates uncertainties about those changes by reporting results in the form of (discrete) distributions.

\section{Data-Only Bayesian Network Model}

Instead of using expert elicitation, a Bayesian network can be parameterized using data alone. Data collected for the 30 EUSE Northeast watersheds can be discretized and organized according to model-structure-specified data tables (table $4 B$ for Hydrology child node; app. 2, tables $2-1 B$ to $2-6 B$ for remaining child nodes). Converting these data tables to probabilities creates the CPTs required to specify a dataonly Bayesian network model. For the sake of comparison, this data-only model (fig. 2-2) was evaluated. Functionally, creating such a Bayesian network requires updating an uninformed prior distribution with the dataset, such that the posterior model reflects only information from the data.

A major problem with using a data-only approach for this model is the small sample size ( 30 watersheds, per CPT) relative to the large number of model parameters (378 $\theta_{i}$ probabilities to define, total). Because of this disparity, many of the variable-state combinations the model attempts to characterize are not represented in the dataset because they were not measured; these include all CPT rows created from data rows in table $4 B$ and appendix 2, tables $2-1 B$ to $2-6 B$ for which $n=0$. One such missing component is the fine-sediment-dominated stream. Streams in the Northeastern United States tend to be geomorphologically coarser, in general, than streams in EUSE studies in other parts of the country, such as in agriculturalrunoff-dominated regions of the Midwest. Because high levels of fine sediments are uncommon in the Northeast, no such stream was observed during EUSE Northeast data collection in this region. Consequently, no data are available to characterize any of the CPT probability rows conditional on fine-sedimentdominated habitat when Habitat is acting as a parent node (a total of $81 \theta_{i}$ probabilities, created from data tables $2-3 B$ to $2-5 B$ in app. 2) nor any of the CPT for the Habitat child node (18 $\theta_{i}$ probabilities, created from data table $2-1 B$ in app. 2 ), even though expert biologists emphasized the role of fine sediments in degrading the macroinvertebrate condition. Because of the lack of data, the Habitat node in the data-only model never changes state from 100 percent "coarse."
Similarly, no streams with "low" generic richness (less than 15 genera) were sampled, probably because the EUSE Northeast study did not include watersheds containing more than 77 percent urban land cover. In the Northeast, many of the most urbanized streams have been converted into concrete culverts or underground pipes. These streams were consciously not sampled as part of EUSE because they were not considered natural stream systems. This exclusion resulted in undersampling of highly urbanized streams, which are the ones most likely to have "low" generic richness. Consequently, any CPT cell involving low generic richness as a child or parent state could not be characterized by the data (72 $\theta_{i}$ probabilities, created from data tables $2-3 B$ and $2-6 B$ in app. 2).

In addition to the two systematic data gaps just identified, many of the remaining parent-state combinations have no data equivalent, with random data gaps increasing as node complexity increases. For the BCG node that has three parents with three states each, only 7 of the 27 parent state combinations have available data (app. 2, table 2-6B). This result is observed because (1) some parent state combinations are more likely to occur than others (for example, 10 of 30 Northeast streams had a parent state combination of medium generic richness, high filter-feeder relative abundance, and low $\mathrm{P}+\mathrm{E}$ abundance); and (2) small sample sizes often do not capture rare state combinations.

Paradoxically, the effect of parameterizing a Bayesian network with limited data is an inflated certainty in the marginal distributions of some nodes; for example, Habitat shows 100 percent coarse substrate (fig. 2-2). In addition, when small-sample-sized data are used to calculate CPTs, node distributions exhibit extreme responses to evidence propagation. For example, in cases of low urban land cover, the data-only model predictive probability of observing BCG Tier 2 (53.86 percent, fig. 12) is much greater than the expertprior model prediction (22.78 percent, fig. 10), and much less than the expert-prior model prediction in cases of high urban land cover ( 0 percent, fig. 13, relative to 5.69 percent, fig. 11). This result can be traced to the abundance of data-gap-induced CPT zeros in the data-only model, which are probabilistically interpreted as a total certainty of nonoccurrence. This false certainty unrealistically distorts prediction magnitude. Although the direction of change is the same in both models, showing that experts and data agree on the direction of variable relations, available data alone are insufficient to adequately parameterize this model. In this situation, there would be very little confidence in the final model if substantial expert information were not included in the prior distributions. 


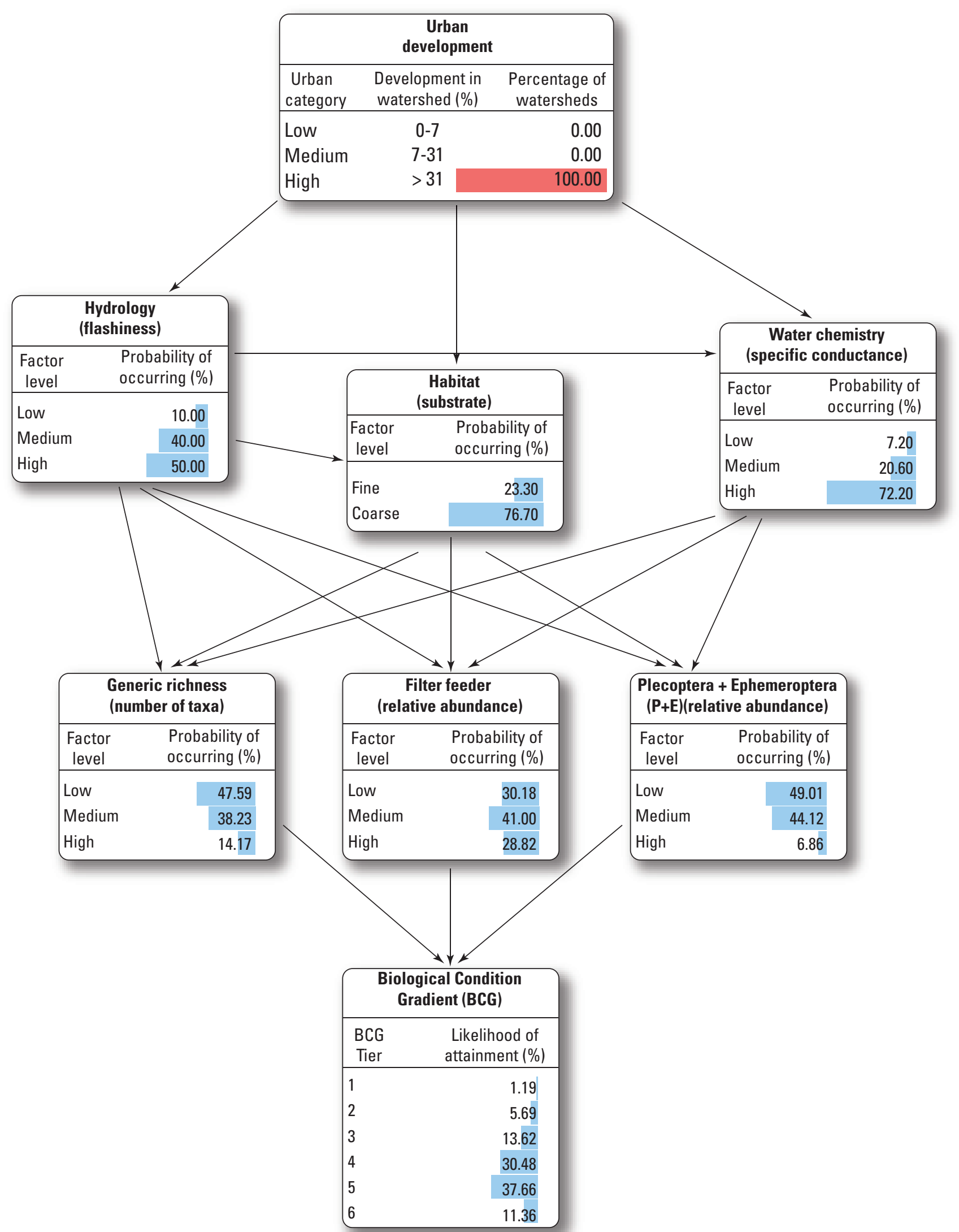

Figure 11. Prior predictive probabilities of occurrence for each level of each node under high urban development. [Probabilities are represented as percentages, numerically and graphically (horizontal colored bars). Factor levels are defined in table 3.] 


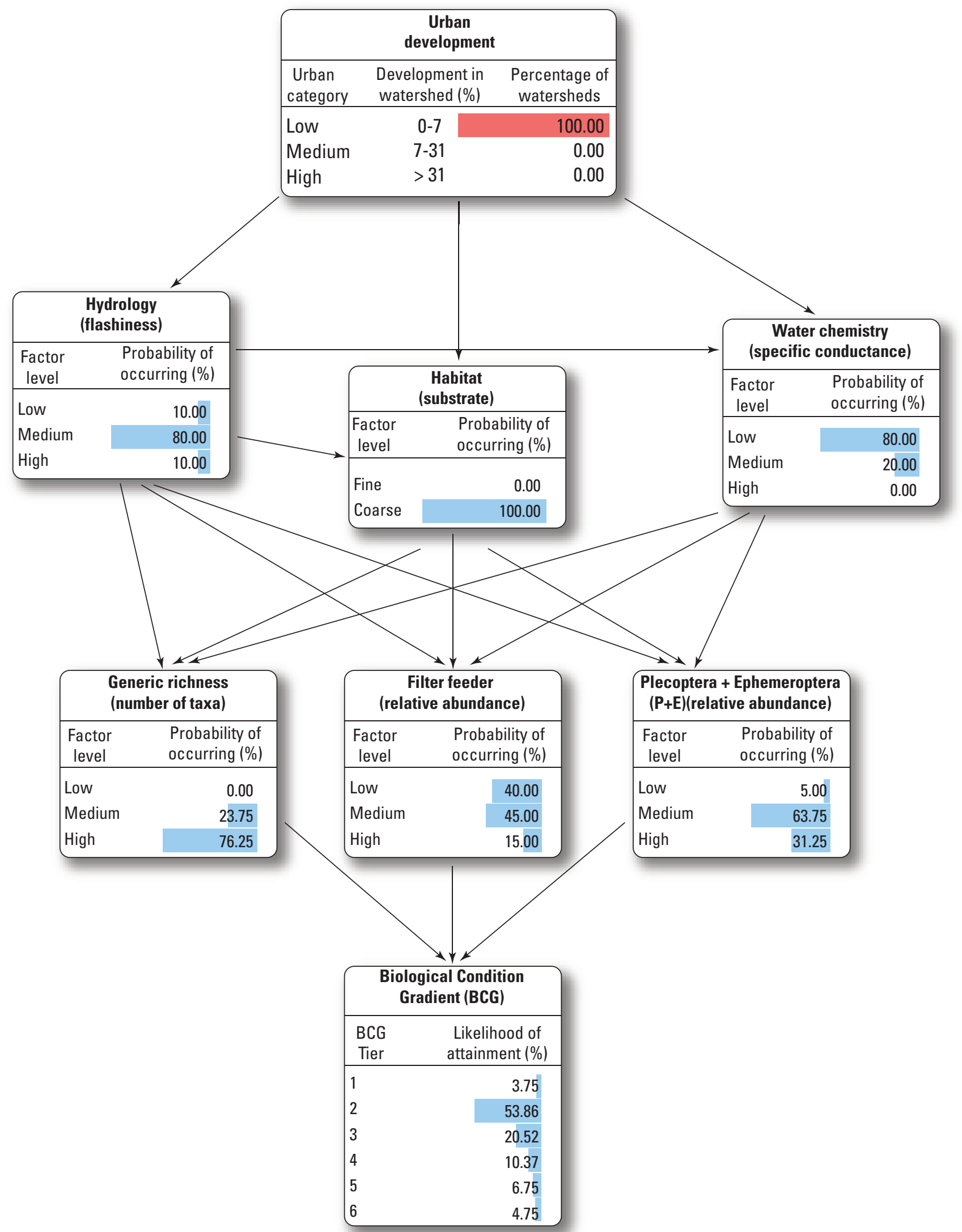

Figure 12. Data-only predictive probabilities of occurrence for each level of each node under low urban development. [Probabilities are represented as percentages, numerically and graphically (horizontal colored bars). Factor levels are defined in table 3.] 


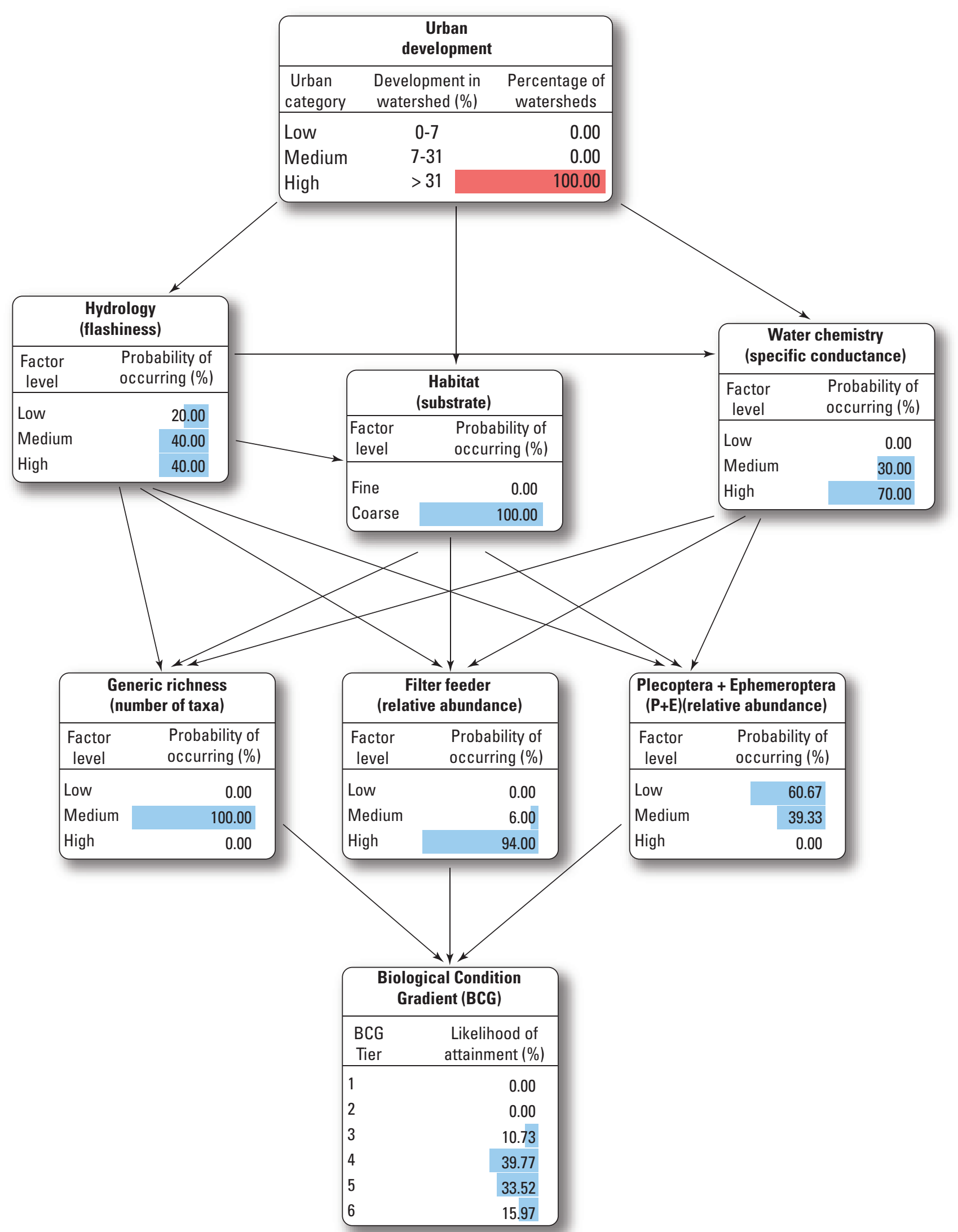

Figure 13. Data-only predictive probabilities of occurrence for each level of each node under high urban development. [Probabilities are represented as percentages, numerically and graphically (horizontal colored bars). Factor levels are defined in table 3.] 


\section{Posterior Bayesian Network Model}

Using Dirichlet-multinomial updating, as described earlier, the expert-elicited prior model was updated with the EUSE Northeast data to create the posterior model. Posterior conditional probability tables, which are a weighted average of prior information and data, are calculated for every child node in the model (tables $4 C$ and app. 2, tables $2-1 C$ to $2-6 C$ ). To evaluate relations described by the posterior model, it is easier to interactively manipulate the Bayesian network than to interpret the static, multidimensional series of CPTs. By entering the hypothetical evidence about each node in turn, system trends can be more clearly understood than from discrete tables of numbers. For example, as specific conductance increases, generic richness decreases, filter feeder relative abundance increases, and $\mathrm{P}+\mathrm{E}$ relative abundance decreases. This means that as water quality becomes impaired, macroinvertebrate communities become less diverse because the sensitive taxa are lost and the overall community becomes more contaminant-tolerant. The posterior Bayesian network also quantifies the relative magnitudes of system changes. For example, flashiness seems to affect filter-feeder relative abundance more than generic richness.

To investigate the potential effect of applying best management practices (BMPs) to urban landscapes, a technique known as causal intervention modeling (Pearl, 2000) is used. Thus far, the posterior network as described has been used to predict states of system variables, conditional upon observing one of the variables. For example, if it is observed that a stream is located in a watershed with high urban land cover, the posterior Bayesian network predicts a 24.46 percent likelihood of that stream achieving BCG Tier 3 or better biological condition (fig. 14). However, if the objective is to use this model to predict the likelihood of achieving a "good" biological condition as a result of a management action (such as an implemented BMP), this action is no longer a simple observation of the system, but rather, an intervention to the system. To model such an intervention to a particular node, all arrows pointing to that node must be removed to show that parent nodes are no longer predicting the value of that child node; instead, the external intervention is now determining the childnode state. The statistics of how those parent nodes would have likely affected that child node are no longer relevant because a definitive intervention, further down the causal chain of events, is being simulated instead.

In the case of modeling effects of urban development, ideally, BMP nodes would be explicitly included, and the effects of those BMPs on the hydrology, habitat, and water quality of a system would be quantified. Without specific information on those relations, at the very least, it is known that implementation of a BMP would disrupt the relations between urban development and hydrology, habitat, and water quality, which are currently modeled in the absence of BMPs.
Therefore, it is logically appropriate (as well as statistically correct) to remove the arrows to simulate the effects of a BMP on each of these three system drivers, because the relation originally modeled would no longer apply.

In terms of notation, the arrow between Urban development and Hydrology in the model represents the current understanding and quantification of that relation across watersheds having different levels of urban development in the absence of BMPs developed from expert knowledge and collected data. However, managers may want to know which BMPs would ultimately improve the biotic condition of a stream the most and data to assess that question directly are not currently available. It can be assumed that BMPs would change the relation (that is, the arrow) between urban development and hydrology, ideally reducing flashiness relative to a particular level of urban development. Because this new, BMP-mediated relation between urban land cover and hydrology is unknown, it cannot be modeled directly. Instead, the arrow specifying the current relation between Urban development and Hydrology (in the absence of BMPs) is removed, and it is assumed that some BMP is able to reduce flashiness from high to medium. The effects of that hypothetical reduction on the rest of the model can then be investigated. When using the technique just described to simulate the effects of a BMP that reduces flashiness to medium (shown with a horizontal green bar), while still observing high urban land cover (horizontal red bar), the likelihood of achieving BCG Tier 3 or better increases to 28.56 percent (fig. 15) relative to 24.46 percent for an unmanaged watershed with high urban land cover (fig. 14).

Alternately, if a BMP is implemented that reduces specific conductance to "low," while still observing high urban land cover, BCG Tier 3 or better attainment is 56.62 percent likely (fig. 16). If both flashiness and specific conductance are managed, this likelihood increases to 69.69 percent (fig. 17).

Ultimately, managers would likely use this model to determine which manipulation to the system will yield the greatest chance of attaining a "good" BCG tier. Averaging across all possible urban land cover scenarios, BCG attainment probabilities were determined for each possible combination of management actions (affecting one, two, or three stressor nodes), and sorted in table 6 according to the best BCG Tier 3 or better probability achieved. The greatest likelihood of attaining BCG Tier 3 or better (79.55 percent) is obtained by managing to achieve medium flashiness, coarse substrate, and low conductance (rank 1, table 6). Conversely, BCG is least likely to be Tier 3 or better (2.33 percent) if management actions cause high flashiness, fine substrate, and high conductance (rank 47, table 6). 


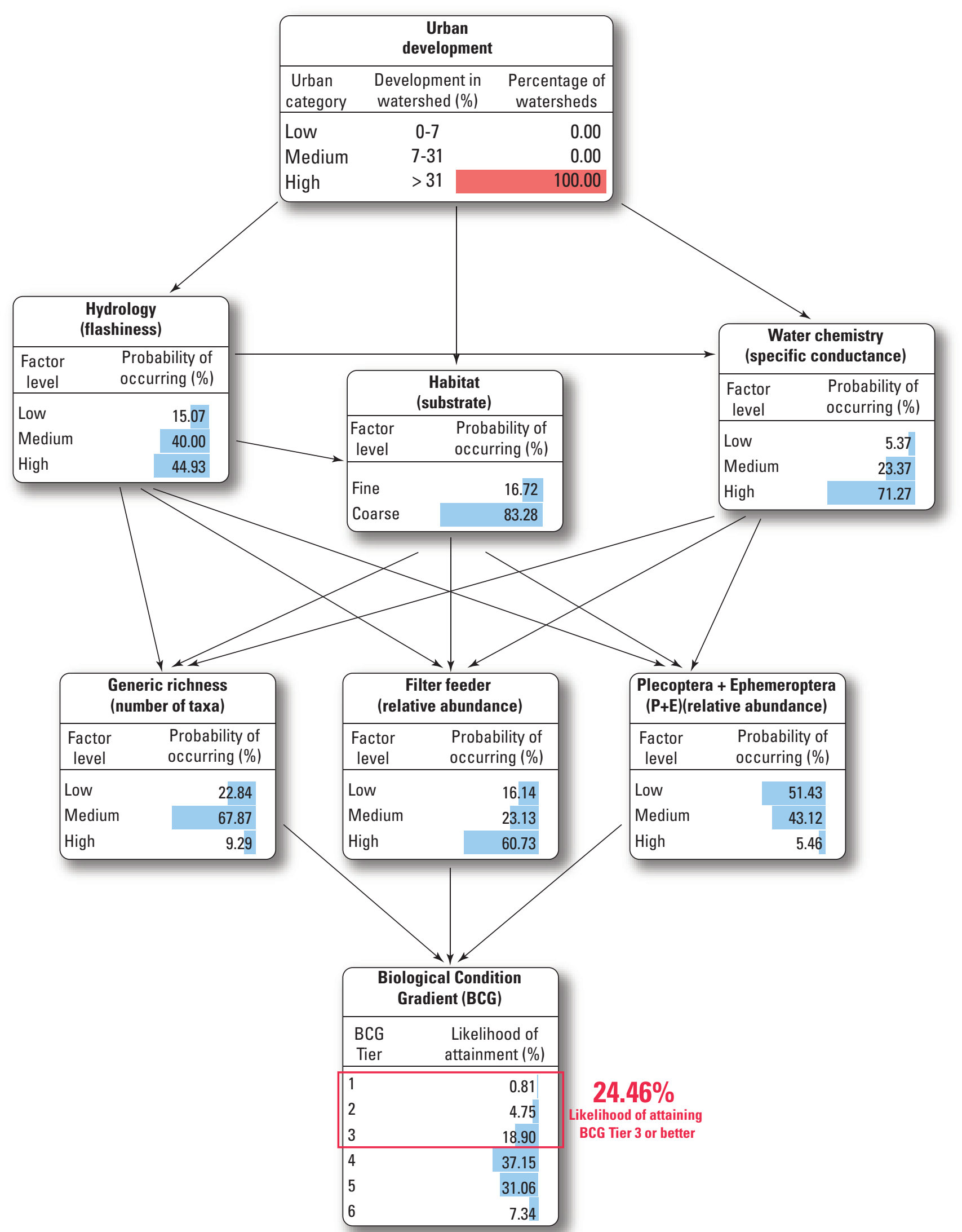

Figure 14. Posterior predictive probabilities of occurrence for each level of each node given an observation of high urban land cover and no management interventions. [Probabilities are represented as percentages, numerically and graphically (horizontal colored bars). Factor levels are defined in table 3.] 


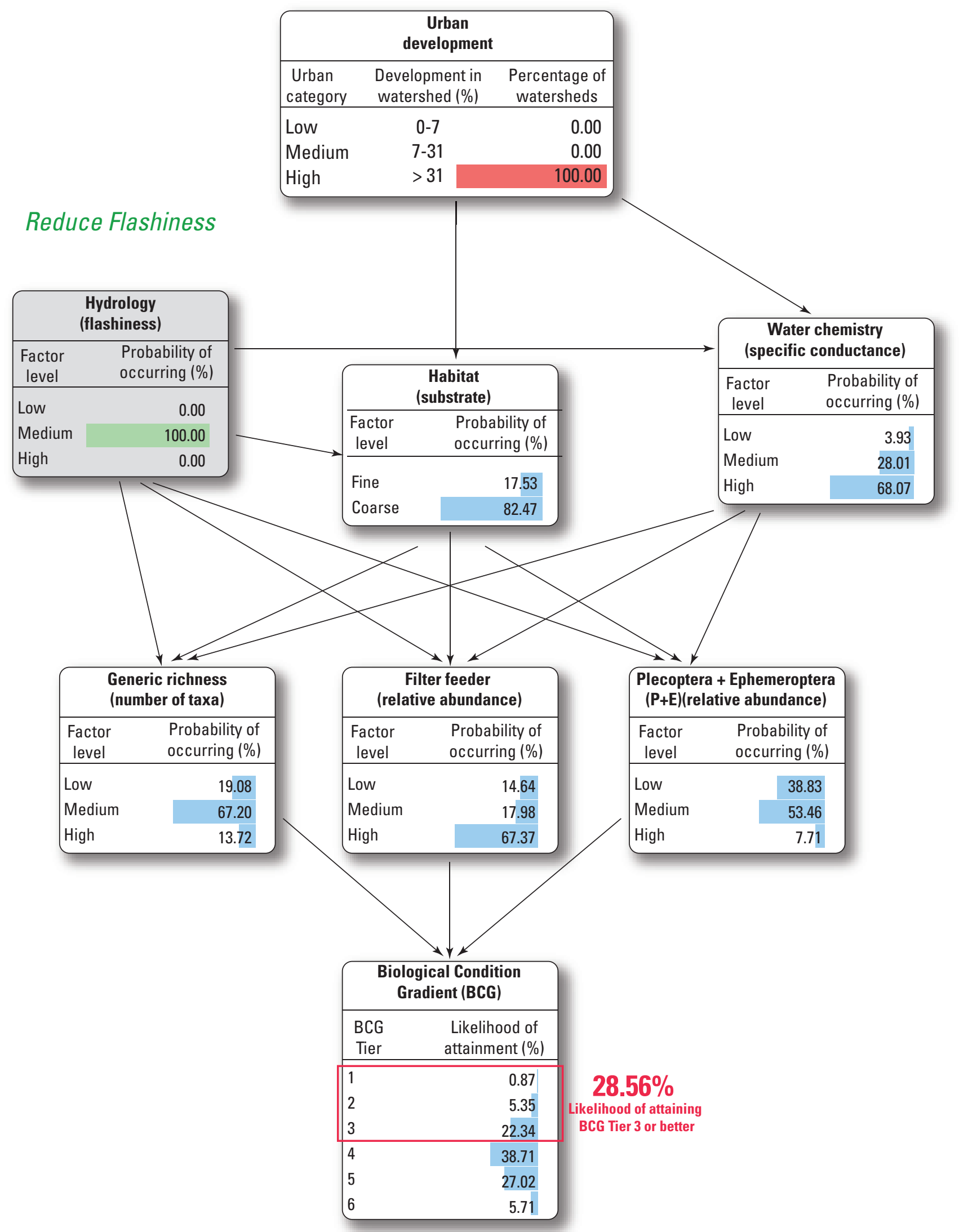

Figure 15. Posterior predictive probabilities of occurrence for each level of each node under causal inference: Managing flashiness to medium, while still observing high urban land cover. [Probabilities are represented as percentages, numerically and graphically (horizontal colored bars). Factor levels are defined in table 3.] 


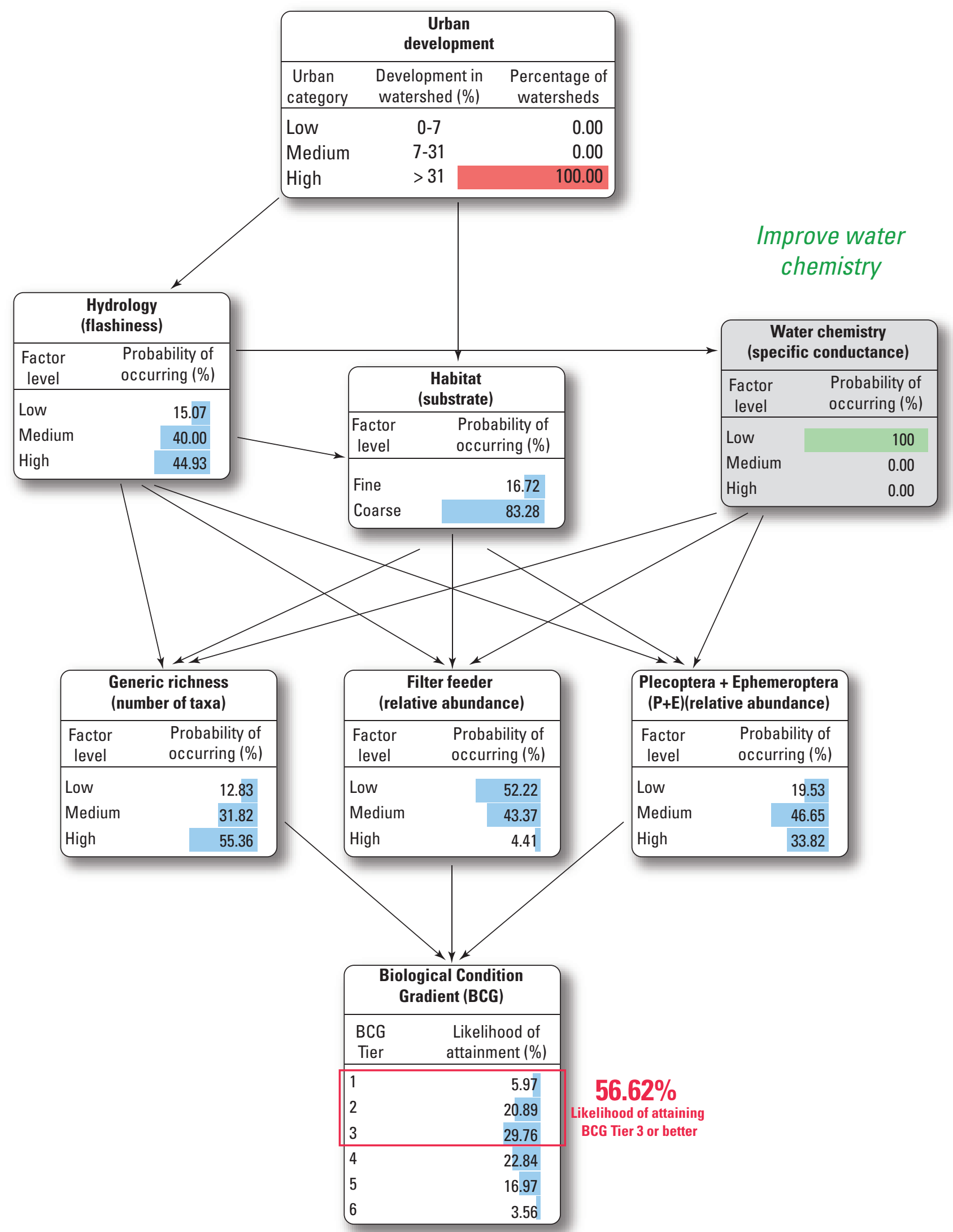

Figure 16. Posterior predictive probabilities of occurrence for each level of each node under causal inference: Managing specific conductance to low, while still observing high urban land cover. [Probabilities are represented as percentages, numerically and graphically (horizontal colored bars). Factor levels are defined in table 3.] 


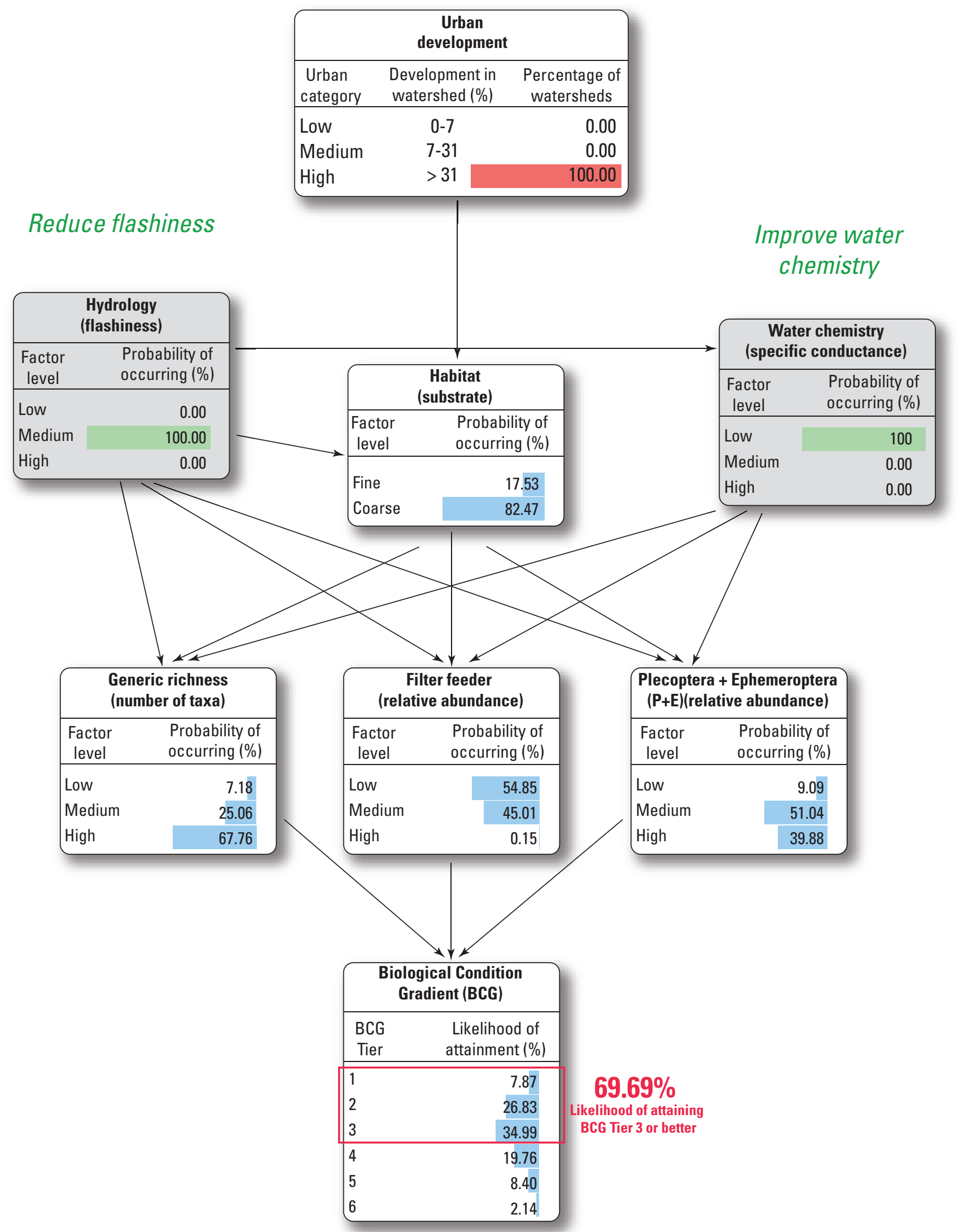

Figure 17. Posterior predictive probabilities of occurrence for each level of each node under causal inference: Managing flashiness to medium and specific conductance to low, while still observing high urban land cover. [Probabilities are represented as percentages, numerically and graphically (horizontal colored bars). Factor levels are defined in table 3.] 
Table 6. Driver causal interventions most likely to improve Biological Condition Gradient (BCG) tier achievement likelihood given unobserved urban land cover state.

[BCG goal is the combined probabilities of attaining BCG Tiers 1, 2, or 3; \%, percent; BMP, best management practice]

\begin{tabular}{|c|c|c|c|c|c|}
\hline Rank & Flashiness & Substrate & Conductance & $\begin{array}{c}\text { BCG goal } \\
\text { (\% achievement) }\end{array}$ & $\begin{array}{l}\text { Nodes } \\
\text { managed }\end{array}$ \\
\hline 1 & Medium & Coarse & Low & 79.55 & 3 \\
\hline 2 & Medium & NoBMP & Low & 73.47 & 2 \\
\hline 3 & NoBMP & Coarse & Low & 68.32 & 2 \\
\hline 4 & NoBMP & NoBMP & Low & 63.39 & 1 \\
\hline 5 & Low & Coarse & Low & 62.36 & 3 \\
\hline 6 & Low & NoBMP & Low & 54.98 & 2 \\
\hline 7 & Medium & Coarse & NoBMP & 50.48 & 2 \\
\hline 8 & High & Coarse & Low & 50.22 & 3 \\
\hline 9 & High & NoBMP & Low & 47.66 & 2 \\
\hline 10 & Medium & NoBMP & NoBMP & 47.45 & 1 \\
\hline 11 & NoBMP & Coarse & NoBMP & 45.26 & 1 \\
\hline 12 & Low & Coarse & NoBMP & 43.97 & 2 \\
\hline 13 & Low & Coarse & Med & 42.38 & 3 \\
\hline 14 & Low & NoBMP & NoBMP & 38.82 & 1 \\
\hline 15 & Low & NoBMP & Med & 36.65 & 2 \\
\hline 16 & Medium & Coarse & Med & 36.23 & 3 \\
\hline 17 & Medium & NoBMP & Med & 34.15 & 2 \\
\hline 18 & High & Coarse & NoBMP & 33.06 & 2 \\
\hline 19 & NoBMP & Coarse & Med & 31.89 & 2 \\
\hline 20 & High & NoBMP & NoBMP & 31.45 & 1 \\
\hline 21 & NoBMP & NoBMP & Med & 29.77 & 1 \\
\hline 22 & Low & Fine & Low & 29.19 & 3 \\
\hline 23 & Low & Coarse & High & 28.66 & 3 \\
\hline 24 & Medium & Coarse & High & 27.92 & 3 \\
\hline 25 & Medium & NoBMP & High & 25.76 & 2 \\
\hline 26 & NoBMP & Coarse & High & 25.66 & 2 \\
\hline 27 & Low & NoBMP & High & 24.94 & 2 \\
\hline 28 & NoBMP & NoBMP & High & 23.59 & 1 \\
\hline 29 & Medium & Fine & Low & 23.35 & 3 \\
\hline 30 & NoBMP & Fine & Low & 22.28 & 2 \\
\hline 31 & High & Coarse & High & 20.05 & 3 \\
\hline 32 & Low & Fine & NoBMP & 19.09 & 2 \\
\hline 33 & High & Coarse & Med & 18.85 & 3 \\
\hline 34 & High & NoBMP & High & 18.68 & 2 \\
\hline 35 & High & NoBMP & Med & 18.04 & 2 \\
\hline 36 & High & Fine & Low & 17.01 & 3 \\
\hline 37 & Medium & Fine & Med & 16.82 & 3 \\
\hline 38 & Low & Fine & Med & 16.64 & 3 \\
\hline 39 & Medium & Fine & NoBMP & 16.57 & 2 \\
\hline 40 & NoBMP & Fine & NoBMP & 14.99 & 1 \\
\hline 41 & NoBMP & Fine & Med & 14.30 & 2 \\
\hline 42 & Low & Fine & High & 11.96 & 3 \\
\hline 43 & High & Fine & NoBMP & 10.21 & 2 \\
\hline 44 & High & Fine & Med & 8.52 & 3 \\
\hline 45 & Medium & Fine & High & 7.96 & 3 \\
\hline 46 & NoBMP & Fine & High & 6.84 & 2 \\
\hline 47 & High & Fine & High & 2.33 & 3 \\
\hline
\end{tabular}




\section{Assessing the Value of a Bayesian Network Approach}

Knowing the relative influence of different changes to an environmental system on its components can be valuable from a management perspective. A manager can use a Bayesian network to assess the relative effect of different model drivers on a desired endpoint, such as BCG tier, to determine which management actions lead to the greatest improvement. In contrast to traditional model prediction, a Bayesian network provides a more holistic approach to endpoint evaluation because it accounts for multiple interactions among variables simultaneously. In addition, the use of Bayesian networks for causal inference incorporates uncertainties that are encoded by the network when predicting driver effects on the endpoint, and results are reported probabilistically for clearer characterization.

\section{Benefits of Using a Bayesian Network for Urban Development Modeling}

Using a Bayesian network to evaluate and rank the potential effects of management interventions has two unique benefits: (1) ability to model nonlinearities and interactions and
(2) model-wide uncertainty incorporation. Many nonlinearities and interactions exist in the process of urbanizing watersheds affecting stream biology. For example, a causal intervention resulting in low flashiness actually predicts a smaller chance of BCG Tier 3 or better attainment than medium flashiness (38.82 percent relative to 47.45 percent; rank 14 and 10, respectively, table 6). Ecologically, this makes sense because low flashiness could be indicative of stagnant flows (such as wetland-dominated streams), which may not be optimal for macroinvertebrates. Moreover, the prior CPTs elicited from experts for the macroinvertebrate metrics show that experts designed the system to work this way (table 7). This form of model construction acknowledges that biotic response is complicated and a function of the interaction between many different drivers, nor is it a simple monotonic relation in which a biotic response always moves in the same direction relative to increasing drivers. A Bayesian network is able to capture this real-world complexity in a way that simple linear models cannot.

Another example of a system complexity modeled effectively by a Bayesian network is the nonlinear effect of conductance on a BCG Tier. Specifically, BCG attainment likelihood does not improve as greatly when managing to reduce specific conductance from high to medium as when managing to reduce specific conductance from medium to low. Managing for low conductance may actually be the management action

Table 7. Excerpts from macroinvertebrate metric prior expert-elicited conditional probability tables.

[Experts often report that Medium flashiness results in the greatest probability of "best" invert metric relative to "low" or "high" flashiness (shown here in frequency units out of 1,000). $<$, less than; \%, percent]

\begin{tabular}{|c|c|c|c|c|c|}
\hline \multicolumn{3}{|l|}{ Given: } & \multicolumn{3}{|c|}{ How would you distribute 1,000 streams? } \\
\hline Flashiness & Substrate & Conductance & \multicolumn{3}{|c|}{ Generic richness } \\
\hline & & & $<15$ & $15-37$ & $38+$ \\
\hline Low & Fine & Low & 300 & 600 & 100 \\
\hline Medium & Fine & Low & 400 & 400 & 200 \\
\hline High & Fine & Low & 500 & 400 & 100 \\
\hline \multirow[t]{2}{*}{ Flashiness } & Substrate & Conductance & \multicolumn{3}{|c|}{ Generic richness } \\
\hline & & & $<15$ & $15-37$ & $38+$ \\
\hline Low & Coarse & High & 350 & 550 & 100 \\
\hline Medium & Coarse & High & 333 & 333 & 333 \\
\hline High & Coarse & High & 590 & 400 & 10 \\
\hline \multirow[t]{2}{*}{ Flashiness } & Substrate & Conductance & \multicolumn{3}{|c|}{$\mathrm{P}+\mathrm{E}$ relative abundance } \\
\hline & & & $<5 \%$ & $5-20 \%$ & $20 \%+$ \\
\hline Low & Coarse & Medium & 100 & 600 & 300 \\
\hline Medium & Coarse & Medium & 10 & 600 & 390 \\
\hline High & Coarse & Medium & 500 & 450 & 50 \\
\hline \multirow[t]{2}{*}{ Flashiness } & Substrate & Conductance & \multicolumn{3}{|c|}{$\mathrm{P}+\mathrm{E}$ relative abundance } \\
\hline & & & $<5 \%$ & $5-20 \%$ & $20 \%+$ \\
\hline Low & Fine & Low & 400 & 550 & 50 \\
\hline Medium & Fine & Low & 500 & 400 & 100 \\
\hline High & Fine & Low & 490 & 500 & 10 \\
\hline
\end{tabular}


most effective at achieving good biological quality; conductance is low in eight of the top nine BCG tier probabilities (table 6). This is similar to a threshold effect in that reducing specific conductance does not appear to help until a threshold point, which the model defines as 139 microsiemens per centimeter. The added complexity is a result of interactions among nodes such that the influence of low conductance is only true at medium or low flashiness and coarse substrate. At other flashiness and substrate states, low conductance does not increase biological condition status substantially; for example, when substrate is fine, low flashiness states are further down the BCG probability ranking list, at ranks $22,29,30,36$ (table 6). These permutations and influences are much more challenging to account for using a non-network model than a network model.

A second benefit of using a Bayesian network is that it accounts for these nonlinearities while simultaneously incorporating uncertainty in its predictions. The BCG output is reported as a probability of attainment to indicate the actual certainty with which model predictions are known. Therefore, a high probability of desirable BCG attainment is difficult to achieve for two reasons:

1. The network of stream ecosystem processes is complex, and it is unrealistic to expect fixes applied to just one node to rectify the entire problem. There are many interacting factors that ultimately affect biological condition, and a higher probability of attainment requires that multiple entities probably need to be managed. The best case scenario the current model can predict is about 80 percent attainment of BCG Tier 3 or better, but this requires managing all three drivers (table 6).

2. There is still much uncertainty and variability that this model is incorporating, including random variability, model simplification error, imperfect knowledge about how the stream ecosystem works, and potentially inaccurate assumptions. Consequently, it is not surprising that the model does not yield "perfect" high-attainment predictions. Given the limitations of what is known and unknown about a particular stream ecosystem, it is still not entirely certain that adjusting the flashiness, substrate, or specific conductance will allow a manager to achieve the desired biological endpoint. In all likelihood, many other factors can affect the endpoint, and the uncertainty in model output is realistically estimated. The level of uncertainty reported is typical for complex environmental questions. Model output quantifies how well the current state of subject-related expertise and data can predict the result of management efforts on the environment. This type of information is more useful and credible than overconfident model results reported with no accompanying uncertainty estimates.

\section{Unresolved Issues}

In addition to exploring the aforementioned benefits of applying a Bayesian network approach, this modeling effort also identified several currently unresolved issues. Primary among these is defining data needs relative to the number of CPT parameters specified by the model structure and discretization scheme. Unlike continuous functions in which a few parameters can describe relations between variables completely, each discrete combination of possible values in a Bayesian network has to be specified separately. This means that even Bayesian networks with few nodes, arrows, and variable categories require many parameters. Although this model format is undeniably an advantage in situations where functional relations between variables are unknown, it also requires large sample sizes in order to be confident of exclusively using data for parameterization. Assessing data requirements is important because data gaps often result in false certainties. This happens because each CPT row represents a discrete multinomial distribution, and the use of only one data point to define that distribution will change the expected values across categories from flat to extreme probabilities. For example, a probability of 0.33 for each of three categories will change to a probability of 0 for two categories and 1 for the third, if the one data point used for distribution specification falls in the third category. This problem is resolved with the use of prior expert knowledge to supplement collected data. To parameterize Bayesian networks with data alone, future studies are needed to establish relations between model confidence and data sample size for a given number of CPT parameters. Until such relations are established, however, Bayesian network users need to be aware of this potentially confounding issue.

A second concern with Bayesian network creation is the transformation of continuous variables into discrete ones. There is no clear method for selecting the optimal number of bins and bin interval endpoints during Bayesian network parameterization. Criteria that can be considered include scientifically based cutoffs, equivalent data frequencies across all bins, equal interval lengths across all bins, and moment matching with data. Further research is needed to better understand the benefits and drawbacks of these potential criteria, evaluate the sensitivity of model outputs to these different modeling choices, and obtain consensus on the appropriate type of method to use for a given modeling situation.

A third issue with using the Bayesian network approach is the lack of consensus on the ideal expert elicitation methodology, particularly for multinomial probabilities. Most probability elicitation literature focuses on assessing single probabilities within a binomial framework (Morgan and Henrion, 1990; Winkler, 2003; Reckhow and others, 2005; O'Hagan and others, 2006). When a discrete variable is composed of more than two categories, specifying a probability ( $p$ ) no longer signifies that the remaining category is assigned the remaining probability $(1-p)$, making a multinomial distribution more complicated to conceptualize than a binomial 
distribution. As such, it is not clear which format for elicitation questions best accesses and represents expert knowledge with more than one degree of freedom.

A related fourth question concerns the elicitation of prior weights. The question of how to best assess the strength of prior knowledge is a topic of active research. Similar to elicitation of the knowledge itself, the goal of prior weight determination is to find a method that best captures expert certainty; however, it is unclear which criteria should be used to make that assessment. Other uncertainties include (1) the scale at which weights are most reliably appraised (CPT cell, row, or entire node), and (2) how to minimize or correct for known human biases (in interpreting extremes, anchoring judgments, and so forth). It is unknown what contributes to the variability in reported weights for a given set of knowledge and how to minimize that variability.

\section{Conclusions}

This effort of Bayesian network model development and updating for the Northeast accomplished three major objectives. First, an innovative method of modeling multiple ecological compartments was devised and implemented. Second, this multicompartment system was linked to a management context with a meaningful endpoint. Third, the effects of potential management interventions on this explicitly defined biological standard were evaluated. Bayesian network analysis also filled many previous data-analysis voids by (1) utilizing full-system-level parameterization and understanding, (2) thoroughly characterizing uncertainty, (3) integrating multiple sources of information, (4) supplementing insufficient data sample size, and (5) allowing customizing the model to fit data structure and problem context by means of the flexibility of Bayesian modeling assumptions.

Specifically, the posterior Bayesian network model demonstrated the utility of incorporating existing expert knowledge as a means of addressing data gaps. The network format of a conceptual model is a useful way to visualize and communicate understanding about how a system works. A Bayesian network goes a step further and quantifies that understanding using all available information. Using the BCG as a model and management endpoint allows for a standardized interpretation of biological condition. Predicting BCG using a probabilistic approach allows one to represent model output realistically and aids decision makers in risk assessment.

Causal inference demonstrates the predictive difference between observing a variable and manipulating a variable. From this evaluation it was learned that managing for low rather than medium conductance is vital to achieving a healthier stream. Overall, however, this model confirmed that there is a complex, nonlinear, interacting set of relations between stressors, and that these complexities are well-modeled using a Bayesian network. The complexity of the system is so great that this model cannot predict any more than about an 80-percent certainty of achieving BCG objectives, even if all three major stressors are managed.

From the model results presented herein, it can be concluded that information from experts and collected data are in agreement on the general directions of change imposed by urban development on hydrology, habitat, water quality, and the biological condition of a stream. In both the expert and data-only models and, hence, in the posterior model, higher urban development levels increased the certainty of observing flashy, fine-substrate-dominated, high conductance streams with poor biological condition. The magnitudes of these system changes relative to each other remain uncertain, however, and require further study.

In reinterpreting the Northeast portion of the EUSE dataset, this Bayesian network quantifies the simultaneous influences of multiple aspects of urban development on macroinvertebrate condition while also quantifying the uncertainty in those relations. Using this framework provides the ability to think of these relations between variables in terms of an interactive cause-and-effect network rather than a list of model parameters with error bars. The effects of a change in a variable on all other nodes in the system can now be modeled, enabling multidimensional data interpretation in a manner not previously possible. In addition, outcomes are predicted in terms of the probability of attaining each BCG tier, which gives the model user a more realistic understanding of outcome likelihood rather than a pseudo-confident exact prediction. Managers can use this Bayesian network to predict the results of possible actions on biological condition and to make quantitative, risk-based decisions in terms of a meaningful and defined aquatic goal, the BCG tier.

The posterior model shows that, of the three drivers of biological change, management actions that reduce specific conductance to low levels have the greatest likelihood of "good" BCG tier achievement, but not in conjunction with fine substrate and, to a lesser extent, high flashiness. In actuality, the drivers follow complex, nonlinear, interacting relations with biological condition, and more than one driver will likely have to be managed to achieve desired BCG endpoints with greater certainty.

Due to the adaptive nature of Bayes Theorem, as more data are collected for this Northeast stream urban development model, the current posteriors become the new priors and the model is further informed. This flexible construct allows the model to "learn" as more data are incorporated. Using this modeling framework, the process of scientific hypothesis creation and verification can be quantified in a transparent manner. This is a means of clearly presenting which expert prior assumptions were included in a particular iteration of the model, instead of not reporting implicit expert input and erroneously referring to model building as "objective." As more data on the system become available over time, they can override less accurate scientific assumptions and fortify correct ones, leading to a much more systematic implementation of the scientific process. 


\section{References Cited}

Barbour, M.T., Gerritsen, J., Snyder, B.D., and Stribling, J.B., 1999, Rapid bioassessment protocols for use in streams and wadeable rivers - Periphyton, benthic macroinvertebrates, and fish ( $2 \mathrm{~d}$ ed.): U.S. Environmental Protection Agency, Office of Water, EPA 841-B-99-002.

Bayes, T., 1763, An essay towards solving a problem in the Doctrine of Chances. By the late Rev. Mr. Bayes, F.R.S. communicated by Mr. Price, in a letter to John Canton, A.M.F.R.S.: Philosophical transactions, v. 53, p. 370-418.

Beck, M.B., 2005, Vulnerability of water quality in intensively developing urban watersheds: Environmental modelling \& software, v. 20, no. 4, p. 381-400.

Booth, D.B., 1990, Stream-channel incision following drainage-basin urbanization: Journal of the American Water Resources Association, v. 26, no. 3, p. 407-417.

Clemen, R.T., 1991, Making hard decisions_-An introduction to decision analysis: Boston, Mass., PWS-Kent Publishing.

Cowell, R.G., Dawid, A.P., Lauritzen, S.L., and Spiegelhalter, D.J., 1999, Probabilistic networks and expert systems: New York, Springer-Verlag.

Cuffney, T.F., Brightbill, R.A., May, J.T., and Waite, I.A., 2010, Responses of benthic macroinvertebrates to environmental changes associated with urbanization in nine metropolitan areas: Ecological Applications v. 20, p. 1384-1401.

Cuffney, T.F., and Falcone, J.A., 2008, Derivation of nationally consistent indices representing urban intensity within and across nine metropolitan areas of the conterminous United States: U.S. Geological Survey Scientific Investigations Report 2008-5095, 36 p.

Davies, S.P., and Jackson, S.K., 2006, The biological condition gradient - A descriptive model for interpreting change in aquatic ecosystems: Ecological Applications, v. 16, no. 4, p. $1251-1266$.

Fitzpatrick, F.A., Waite, I.A., Arconte, P.J.D., Meador, M.R., Maupin, M.A., and Gurtz, M.E., 1998, Revised methods for characterizing stream habitat in the National Water-Quality Assessment Program: U.S. Geological Survey WaterResources Investigations Report 98-4052, 67 p.

Gerritsen, J., 2008, The Biological Condition GradientModel development and calibration: U.S. Environmental Protection Agency, Office of Science and Technology, prepared by Tetra Tech, Inc., Owings Mills, Md.

Giddings, E.M.P., Bell, A.H., Beaulieu, K.M., Cuffney, T.F., Coles, J.F., Brown, L.R., Fitzpatrick, F.A., Falcone, James, Sprague, L.A., Bryant, W.L., Peppler, M.C., Stephens, Cory, and McMahon, Gerard, 2009, Selected physical, chemical, and biological data used to study urbanizing streams in nine metropolitan areas of the United States, 1999-2004. U.S. Geological Survey Data Series 423, 11 p. + data tables.
Haase, D., 2003, Holocene floodplains and their distribution in urban areas-Functionality indicators for their retention potentials: Landscape and Urban Planning, v. 66, no. 1, p. 5-18.

Herlihy, A.T., Paulsen, S.G., Sickle, J.V., Stoddard, J.L., Hawkins, C.P., and Yuan, L.L., 2008, Striving for consistency in a national assessment - The challenges of applying a reference-condition approach at a continental scale: Journal of the North American Benthological Society, v. 27, no. 4 , p. $860-877$.

Hugin Expert A/S, 2008, Hugin API reference manual, version 7.0. released September 9, 2008: Aalborg, Denmark, 200 p.

Jacobson, M.Z., 2001, GATOR-GCMM-A global- through urban-scale air pollution and weather forecast model, 1. Model design and treatment of subgrid soil, vegetation, roads, rooftops, water, sea ice, and snow: Journal of Geophysical Research, v. 106, no. D6, p. 5385-5401.

Jensen, F.V., and Nielsen, T.D., 2007, Bayesian networks and decision graphs (2d ed.): Springer, New York, 447 p.

Jones, R.C., and Clark, C.C., 1987, Impact of watershed urbanization on stream insect communities: Journal of the American Water Resources Association, v. 23, no. 6, p. 1047-1055.

Kashuba, R.O., 2010, Bayesian methods to characterize uncertainty in predictive modeling of the effect of urbanization on aquatic ecosystems: Durham, N.C., Duke University, Ph.D. dissertation, 464 p., accessed March 7, 2012, at http://hdl.handle.net/10161/2366.

Kashuba, Roxolana, Cha, YoonKyung, Alameddine, Ibrahim, Lee, Boknam, and Cuffney, T.F., 2010, Multilevel hierarchical modeling of benthic macroinvertebrate responses to urbanization in nine metropolitan regions across the conterminous United States: U.S. Geological Survey Scientific Investigations Report 2009-5243, 88 p.

Klein, R.D., 1979, Urbanization and stream quality impairment: Journal of the American Water Resources Association, v. 15, no. 4, p. 948-963.

Lunn, D.J., Thomas, A., Best, N., and Spiegelhalter, D., 2000, WinBUGS - A Bayesian modelling framework - Concepts, structure, and extensibility: Statistics and Computing, v. 10, no. 4 , p. 325-337.

McMahon, Gerard, Bales, J.D., Coles, J.F., Giddings, E.M.P., and Zappia, Humbert, 2003, Use of stage data to characterize hydrologic conditions in an urbanizing environment: Journal of the American Water Resources Association, v. 39, no. 6 , p. $1529-1546$.

Morgan, M.G., and Henrion, Max, 1990, Uncertainty-A guide to dealing with uncertainty in quantitative risk and policy analysis: Cambridge, U.K., Cambridge University Press, $332 \mathrm{p}$. 
Moulton, S.R., II, Kennen, J.G., Goldstein, R.M., and Hambrook, J.A., 2002, Revised protocols for sampling algal, invertebrate, and fish communities as part of the National Water-Quality Assessment Program: U.S. Geological Survey Open-File Report 02-150, 75 p.

Nadkarni, S., and Shenoy, P.P., 2004, A causal mapping approach to constructing Bayesian networks: Decision support systems, v. 38, no. 2, p. 259-281.

O’Hagan, Anthony, Buck, C.E., Daneshkhah, Alireza, Eiser, J.R., Garthwaite, P.H., Jenkinson, D.J., Oakley, J.E., and Rakow, Tim, 2006, Uncertain judgments-Eliciting experts' probabilities: Chichester, U.K., John Wiley \& Sons.

Pearl, Judea, 1988, Probabilistic reasoning in intelligent systems-Networks of plausible inference: Morgan Kaufmann, San Francisco, Calif., 552 p.

Pearl, Judea, 2000, Causality—Models, reasoning, and inference: Cambridge University Press, New York, 384 p.

Poff, N.L., Allan, J.D., Bain, M.B., Karr, J.R., Prestegaard, K.L., Richter, B.D., Sparks, R.E., and Stromberg, J.C., 1997, The natural flow regime: BioScience v. 47 , no. 11 , p. 769-784.

Reckhow, K.H., 1999, Water quality prediction and probability network models: Canadian Journal of Fisheries and Aquatic Sciences, v. 56, no. 7, p. 1150-1158.

Reckhow, K.H., Arhonditsis, G.B., Kenney, M.A., Hauser, L., Tribo, J., Wu, C., Elcock, K.J., Steinberg, L.J., Stow, C.A., and McBride, S.J., 2005, A predictive approach to nutrient criteria: Environmental Science \& Technology, v. 39, no. 9, p. 2913-2919.

Shelton, A.D., and Blocksom, K.A., 2004, A review of biological assessment tools and biocriteria for rivers and streams in New England States: U.S. Environmental Protection Agency report, EPA/600/R-04/168 [variously paged].

Snook, Hilary, Davies, S.P., Gerritsen, Jeroen, Jessup, B.K., Langdon, Richard, Neils, David, and Pizutto, Ernest, 2007, The New England Wadeable Stream Survey (NEWS) Development of common assessments in the framework of the Biological Condition Gradient: U.S. Environmental Protection Agency Office of Science and Technology, Office of Watersheds, accessed March 8, 2012, at http://www.epa. gov/region1/lab/pdfs/NEWSfinalReport_August2007.pdf.

Spetzler, C.S., and von Holstein, C.A.S.S., 1975, Probability encoding in decision analysis: Management Science, v. 22, no. 3, p. 340-358.

Spiegelhalter, D.J., Harris, N.L., Bull, K., and Franklin, R.C.G., 1994, Empirical evaluation of prior beliefs about frequencies-Methodology and a case study in congenital heart disease: Journal of the American Statistical Association, v. 89 , no. 426, p. 435-443.
Sprague, L.A., Zuelig, R.E., and Dupree, J.A., 2006, Effects of Urbanization on Stream Ecosystems in the South Platte River Basin, Colorado and Wyoming: U.S. Geological Survey Scientific Investigations Report 2006-5101-A, 139 p.

Tate, C., Cuffney, T., McMahon, G., Giddings, E., Coles, J., and Zappia, H., 2005, Use of an urban intensity index to assess urban effects on streams in three contrasting environmental settings, in Brown, L.R., Gray, R.H., Hughes, R.M., and Meador, M.R., eds., Effects of Urbanization on Stream Ecosystems, American Fisheries Society, Symposium 47: Bethesda, Md., p. 291-315.

Trimble, S.W., 1997, Contribution of stream channel erosion to sediment yield from an urbanizing watershed: Science, v. 278 , no. 5342 , p. $1442-1444$.

U.S. Environmental Protection Agency, 2006, Wadeable streams assessment-A collaborative survey of the Nation's streams: U.S. Environmental Protection Agency Office of Research and Development, Office of Water, EPA 841-B06-002, $113 \mathrm{p}$.

Uusitalo, L., 2007, Advantages and challenges of Bayesian networks in environmental modeling: Ecological Modelling, v. 203, nos. 3-4, p. 312-318.

Van Metre, P.C., Mahler, B.J., and Furlong, E.T., 2000, Urban sprawl leaves its PAH signature: Environmental Science \& Technology, v. 34, no. 19, p. 4064-4070.

Winkler, R.L., 1967, The assessment of prior distributions in Bayesian analysis: Journal of the American Statistical Association, v. 67, p. 776-800.

Winkler, R.L., 2003, An introduction to Bayesian inference and decision ( $2 \mathrm{~d}$ ed.): Gainesville, Fla., Probabilistic Publishing.

Wolman, M.G., and Schick, A.P., 1967, Effects of construction on fluvial sediment, urban and suburban areas of Maryland: Water Resources Research, v. 3, no. 2, p. 451-464.

Yoder, C., and Barbour, M., 2009, Critical technical elements of state bioassessment programs-A process to evaluate program rigor and comparability: Environmental Monitoring and Assessment, v. 150, p. 31-42.

Yoder, C.O., Miltner, R.J., and White, D., 1999, Assessing the status of aquatic life designated uses in urban and suburban watersheds, in Everson, A., Minamyer, S., Dye, J., Heimbrock, P., and Wilson, S., eds., National Conference on Retrofit Opportunities for Water Resource Protection in Urban Environments: U.S. Environmental Protection Agency, EPA/625/R-99/002, p. 16-28. 


\section{Appendix 1. Distribution Forms for Bayesian Updating}

Dirichlet prior distribution:

$$
\begin{gathered}
p\left(\theta_{1}, \theta_{2}, \theta_{3}\right) \propto \theta_{1}^{\alpha_{1}-1} \theta_{2}^{\alpha_{2}-1} \theta_{3}^{\alpha_{3}-1} \\
\sum_{i=1}^{3} \alpha_{i}=\alpha_{0}
\end{gathered}
$$

where

$\theta_{1}-\theta_{3} \quad$ are the probabilities of a sample belonging to categories $1-3$, respectively;

$\alpha_{1}-\alpha_{3} \quad$ are coefficients describing the possible values of $\theta_{1}-\theta_{3}$, respectively; and

$\alpha_{0} \quad$ is the sum of $\alpha_{1}-\alpha_{3}$, also known as total prior weight or equivalent sample size.

Solve for prior weight, $\alpha_{0}$, by means of equations for Dirichlet expected value and Dirichlet expected variance:

$$
\begin{gathered}
E\left[\theta_{i}\right]=\frac{\alpha_{i}}{\alpha_{0}} \\
\operatorname{var}\left[\theta_{i}\right]=\frac{\alpha_{i}\left(\alpha_{0}-\alpha_{i}\right)}{\alpha_{0}{ }^{2}\left(\alpha_{0}+1\right)} \\
\alpha_{0}=\frac{E\left[\theta_{i}\right]-E\left[\theta_{i}\right]^{2}-\operatorname{var}\left[\theta_{i}\right]}{\operatorname{var}\left[\theta_{i}\right]}
\end{gathered}
$$

Multinomial likelihood function:

$$
\begin{gathered}
p\left(x_{1}, x_{2}, x_{3} \mid \theta_{1}, \theta_{2}, \theta_{3}\right) \propto \theta_{1}^{x_{1}} \theta_{2}^{x_{2}} \theta_{3}^{x_{3}} \\
\sum_{i=1}^{3} x_{i}=n
\end{gathered}
$$

where

$x_{1}-x_{3} \quad$ are the counts of samples belonging to each of three categories, respectively,

$n \quad$ is the sum of all counts (that is, total sample size), and

$\theta_{1}-\theta_{3} \quad$ are the probabilities of a sample belonging to categories $1-3$, respectively.

Dirichlet posterior distribution:

$$
\begin{gathered}
p\left(\theta_{1}, \theta_{2}, \theta_{3} \mid x_{1}, x_{2}, x_{3}\right) \propto \theta_{1}^{x_{1}+\alpha_{1}-1} \theta_{2}^{x_{2}+\alpha_{2}-1} \theta_{3}^{x_{3}+\alpha_{3}-1} \\
\sum_{i=1}^{3}\left(\alpha_{i}+x_{i}\right)=\alpha_{0}+n
\end{gathered}
$$

where

$\theta_{1}-\theta_{3} \quad$ are the probabilities of a sampled basin belonging to categories 1-3, respectively;

$\alpha_{1}-\alpha_{3} \quad$ are prior coefficients describing the possible values of $\theta_{1}-\theta_{3}$, respectively;

$\alpha_{0} \quad$ is the sum of $\alpha_{1}-\alpha_{3}$, also known as total prior weight or equivalent sample size;

$x_{1}-x_{3} \quad$ are the counts of sampled basins belonging to each of three categories, respectively; and

$n \quad$ is the sum of all counts (that is, total sample size). 


\section{Appendix 2. Supplemental Prior and Posterior Conditional Probability Tables, Data Tables, and Bayesian Network Diagrams}

Table 2-1. "Habitat" node prior conditional probability table and prior weight $\left(\alpha_{0}\right)$, data table, and posterior conditional probability table and posterior weights $\left(\alpha_{0}+n\right)$.

\section{A. Prior conditional probability table}

[Substrate categories in probability units and $\alpha_{0}$ in equivalent data points; rows selected for prior weight elicitation are highlighted]

\begin{tabular}{lllcc}
\hline \multirow{2}{*}{$\begin{array}{c}\text { Urban land } \\
\text { cover }\end{array}$} & Flashiness & \multicolumn{3}{c}{ Dominant substrate } \\
\cline { 3 - 5 } & & Fine & Coarse & $\boldsymbol{\alpha}_{0}$ \\
\hline Low & Low & 0.15 & 0.85 & 7.41 \\
Low & Medium & 0.10 & 0.90 & 7.41 \\
Low & High & 0.05 & 0.95 & 7.41 \\
Medium & Low & 0.25 & 0.75 & 7.41 \\
Medium & Medium & 0.17 & 0.83 & 7.41 \\
Medium & High & 0.12 & 0.88 & 7.41 \\
High & Low & 0.40 & 0.60 & 7.41 \\
High & Medium & 0.27 & 0.73 & 7.41 \\
High & High & 0.17 & 0.83 & 7.41 \\
\hline
\end{tabular}

\section{B. Data table}

[Substrate counts and $n$ total number of samples per parent state combination]

\begin{tabular}{llccc}
\hline \multirow{2}{*}{$\begin{array}{c}\text { Urban land } \\
\text { cover }\end{array}$} & Flashiness & \multicolumn{3}{c}{ Dominant substrate } \\
\cline { 3 - 5 } & & Fine & Coarse & $\boldsymbol{n}$ \\
\hline Low & Low & 0 & 1 & 1 \\
Low & Medium & 0 & 8 & 8 \\
Low & High & 0 & 1 & 1 \\
Medium & Low & 0 & 1 & 1 \\
Medium & Medium & 0 & 5 & 5 \\
Medium & High & 0 & 4 & 4 \\
High & Low & 0 & 2 & 2 \\
High & Medium & 0 & 4 & 4 \\
High & High & 0 & 4 & 4 \\
\hline
\end{tabular}

C. Posterior conditional probability table

[Substrate categories in probability units and $\alpha_{0}+n$ in equivalent data points]

\begin{tabular}{llccc}
\hline \multirow{2}{*}{$\begin{array}{c}\text { Urban land } \\
\text { cover }\end{array}$} & Flashiness & \multicolumn{3}{c}{ Dominant substrate } \\
\cline { 3 - 5 } & & Fine & Coarse & $\boldsymbol{\alpha}_{0}+\boldsymbol{n}$ \\
\hline Low & Low & 0.132 & 0.868 & 8.41 \\
Low & Medium & 0.048 & 0.952 & 15.41 \\
Low & High & 0.044 & 0.956 & 8.41 \\
Medium & Low & 0.220 & 0.780 & 8.41 \\
Medium & Medium & 0.102 & 0.898 & 12.41 \\
Medium & High & 0.078 & 0.922 & 11.41 \\
High & Low & 0.315 & 0.685 & 9.41 \\
High & Medium & 0.175 & 0.825 & 11.41 \\
High & High & 0.110 & 0.890 & 11.41 \\
\hline
\end{tabular}


Table 2-2. "Water quality" node prior conditional probability table and prior weight $\left(\alpha_{0}\right)$, data table, and posterior conditional probability table and posterior weights $\left(\alpha_{0}+n\right)$.

\section{A. Prior conditional probability table}

[Specific conductance categories in probability units and $\alpha_{0}$ in equivalent data points; rows selected for prior weight elicitation are highlighted]

\begin{tabular}{lllccc}
\hline \multirow{2}{*}{$\begin{array}{c}\text { Urban land } \\
\text { cover }\end{array}$} & Flashiness & \multicolumn{4}{c}{ Specific conductance at low base flow } \\
\cline { 3 - 6 } & & Low & Medium & High & $\boldsymbol{\alpha}_{0}$ \\
\hline Low & Low & 0.85 & 0.10 & 0.05 & 14.65 \\
Low & Medium & 0.90 & 0.06 & 0.04 & 14.65 \\
Low & High & 0.95 & 0.04 & 0.01 & 14.65 \\
Medium & Low & 0.20 & 0.50 & 0.30 & 14.65 \\
Medium & Medium & 0.25 & 0.50 & 0.25 & 14.65 \\
Medium & High & 0.30 & 0.50 & 0.20 & 14.65 \\
High & Low & 0.02 & 0.23 & 0.75 & 14.65 \\
High & Medium & 0.05 & 0.22 & 0.73 & 14.65 \\
High & High & 0.10 & 0.19 & 0.71 & 14.65 \\
\hline
\end{tabular}

\section{B. Data table}

[Specific conductance counts and $n$ total number of samples per parent state combination]

\begin{tabular}{llcccc}
\hline \multirow{2}{*}{$\begin{array}{c}\text { Urban land } \\
\text { cover }\end{array}$} & Flashiness & \multicolumn{4}{c}{ Specific conductance at low base flow } \\
\cline { 3 - 6 } & & Low & Medium & High & $\boldsymbol{n}$ \\
\hline Low & Low & 0 & 1 & 0 & 1 \\
Low & Medium & 7 & 1 & 0 & 8 \\
Low & High & 1 & 0 & 0 & 1 \\
Medium & Low & 0 & 1 & 0 & 1 \\
Medium & Medium & 1 & 3 & 1 & 5 \\
Medium & High & 1 & 1 & 2 & 4 \\
High & Low & 0 & 0 & 2 & 2 \\
High & Medium & 0 & 2 & 2 & 4 \\
High & High & 1 & 1 & 3 & 4 \\
\hline
\end{tabular}

C. Posterior conditional probability table

[Specific conductance categories in probability units and $\alpha_{0}+n$ in equivalent data points]

\begin{tabular}{llcccc}
\hline \multirow{2}{*}{$\begin{array}{c}\text { Urban land } \\
\text { cover }\end{array}$} & Flashiness & \multicolumn{3}{c}{ Specific conductance at low base flow } \\
\cline { 3 - 5 } & & Low & Medium & High & $\boldsymbol{\alpha}_{0}+n$ \\
\hline Low & Low & 0.796 & 0.158 & 0.047 & 15.65 \\
Low & Medium & 0.891 & 0.083 & 0.026 & 22.65 \\
Low & High & 0.953 & 0.037 & 0.009 & 15.65 \\
Medium & Low & 0.187 & 0.532 & 0.281 & 15.65 \\
Medium & Medium & 0.237 & 0.525 & 0.237 & 19.65 \\
Medium & High & 0.289 & 0.446 & 0.264 & 18.65 \\
High & Low & 0.018 & 0.202 & 0.780 & 16.65 \\
High & Medium & 0.039 & 0.280 & 0.681 & 18.65 \\
High & High & 0.079 & 0.203 & 0.719 & 18.65 \\
\hline
\end{tabular}


Table 2-3. "Generic richness" node prior conditional probability table and prior weight $\left(\alpha_{0}\right)$, data table, and posterior conditional probability table and posterior weights $\left(\alpha_{0}+n\right)$.

\section{A. Prior conditional probability table}

[Richness categories in probability units and $\alpha_{0}$ in equivalent data points; rows selected for prior weight elicitation are highlighted]

\begin{tabular}{lllcccc}
\hline \multirow{2}{*}{ Flashiness } & \multirow{2}{*}{ Substrate } & Conductance & \multicolumn{4}{c}{ Generic richness } \\
\cline { 4 - 6 } & & & Low & Medium & High & $\boldsymbol{\alpha}_{0}$ \\
\hline Low & Fine & Low & 0.300 & 0.600 & 0.100 & 1.97 \\
Low & Fine & Medium & 0.400 & 0.500 & 0.100 & 1.97 \\
Low & Fine & High & 0.600 & 0.390 & 0.010 & 1.97 \\
Low & Coarse & Low & 0.010 & 0.490 & 0.500 & 1.97 \\
Low & Coarse & Medium & 0.100 & 0.350 & 0.550 & 1.97 \\
Low & Coarse & High & 0.350 & 0.550 & 0.100 & 1.97 \\
Medium & Fine & Low & 0.400 & 0.400 & 0.200 & 1.97 \\
Medium & Fine & Medium & 0.500 & 0.400 & 0.100 & 1.97 \\
Medium & Fine & High & 0.700 & 0.299 & 0.001 & 1.97 \\
Medium & Coarse & Low & 0.010 & 0.600 & 0.390 & 1.97 \\
Medium & Coarse & Medium & 0.100 & 0.350 & 0.550 & 1.97 \\
Medium & Coarse & High & 0.333 & 0.333 & 0.333 & 1.97 \\
High & Fine & Low & 0.500 & 0.400 & 0.100 & 1.97 \\
High & Fine & Medium & 0.600 & 0.390 & 0.010 & 1.97 \\
High & Fine & High & 0.900 & 0.099 & 0.001 & 1.97 \\
High & Coarse & Low & 0.300 & 0.600 & 0.100 & 1.97 \\
High & Coarse & Medium & 0.450 & 0.500 & 0.050 & 1.97 \\
High & Coarse & High & 0.590 & 0.400 & 0.010 & 1.97 \\
\hline
\end{tabular}

\section{B. Data table}

[Richness counts and $n$ total number of samples per parent state combination]

\begin{tabular}{|c|c|c|c|c|c|c|}
\hline \multirow{2}{*}{ Flashiness } & \multirow{2}{*}{ Substrate } & \multirow{2}{*}{ Conductance } & \multicolumn{4}{|c|}{ Generic richness } \\
\hline & & & Low & Medium & High & $n$ \\
\hline Low & Fine & Low & 0 & 0 & 0 & 0 \\
\hline Low & Fine & Medium & 0 & 0 & 0 & 0 \\
\hline Low & Fine & High & 0 & 0 & 0 & 0 \\
\hline Low & Coarse & Low & 0 & 0 & 0 & 0 \\
\hline Low & Coarse & Medium & 0 & 1 & 1 & 2 \\
\hline Low & Coarse & High & 0 & 2 & 0 & 2 \\
\hline Medium & Fine & Low & 0 & 0 & 0 & 0 \\
\hline Medium & Fine & Medium & 0 & 0 & 0 & 0 \\
\hline Medium & Fine & High & 0 & 0 & 0 & 0 \\
\hline Medium & Coarse & Low & 0 & 1 & 7 & 8 \\
\hline Medium & Coarse & Medium & 0 & 6 & 0 & 6 \\
\hline Medium & Coarse & High & 0 & 3 & 0 & 3 \\
\hline High & Fine & Low & 0 & 0 & 0 & 0 \\
\hline High & Fine & Medium & 0 & 0 & 0 & 0 \\
\hline High & Fine & High & 0 & 0 & 0 & 0 \\
\hline High & Coarse & Low & 0 & 0 & 2 & 2 \\
\hline High & Coarse & Medium & 0 & 2 & 0 & 2 \\
\hline High & Coarse & High & 0 & 5 & 0 & 5 \\
\hline
\end{tabular}


Table 2-3. "Generic richness" node prior conditional probability table and prior weight $\left(\alpha_{0}\right)$, data table, and posterior conditional probability table and posterior weights $\left(\alpha_{0}+n\right)$.-Continued

C. Posterior conditional probability table

[Richness categories in probability units and $\alpha_{0}+n$ in equivalent data points]

\begin{tabular}{lllcccc}
\hline \multirow{2}{*}{ Flashiness } & \multirow{2}{*}{ Substrate } & Conductance & \multicolumn{4}{c}{ Generic richness } \\
\cline { 4 - 7 } & & & Low & Medium & High & $\boldsymbol{\alpha}_{0}+\boldsymbol{n}$ \\
\hline Low & Fine & Low & 0.300 & 0.600 & 0.100 & 1.97 \\
Low & Fine & Medium & 0.400 & 0.500 & 0.100 & 1.97 \\
Low & Fine & High & 0.600 & 0.390 & 0.010 & 1.97 \\
Low & Coarse & Low & 0.010 & 0.490 & 0.500 & 1.97 \\
Low & Coarse & Medium & 0.050 & 0.426 & 0.525 & 3.97 \\
Low & Coarse & High & 0.174 & 0.777 & 0.050 & 3.97 \\
Medium & Fine & Low & 0.400 & 0.400 & 0.200 & 1.97 \\
Medium & Fine & Medium & 0.500 & 0.400 & 0.100 & 1.97 \\
Medium & Fine & High & 0.700 & 0.299 & 0.001 & 1.97 \\
Medium & Coarse & Low & 0.002 & 0.219 & 0.779 & 9.97 \\
Medium & Coarse & Medium & 0.025 & 0.839 & 0.136 & 7.97 \\
Medium & Coarse & High & 0.132 & 0.736 & 0.132 & 4.97 \\
High & Fine & Low & 0.500 & 0.400 & 0.100 & 1.97 \\
High & Fine & Medium & 0.600 & 0.390 & 0.010 & 1.97 \\
High & Fine & High & 0.900 & 0.099 & 0.001 & 1.97 \\
High & Coarse & Low & 0.149 & 0.298 & 0.553 & 3.97 \\
High & Coarse & Medium & 0.223 & 0.752 & 0.025 & 3.97 \\
High & Coarse & High & 0.167 & 0.830 & 0.003 & 6.97 \\
\hline
\end{tabular}


Table 2-4. "Filter feeder relative abundance" node prior conditional probability table and prior weight $\left(\alpha_{0}\right)$, data table, and posterior conditional probability table and posterior weights $\left(\alpha_{0}+n\right)$.

\section{A. Prior conditional probability table}

[Relative abundance categories in probability units and $\alpha_{0}$ in equivalent data points; rows selected for prior weight elicitation are highlighted]

\begin{tabular}{llllccc}
\hline \multirow{2}{*}{ Flashiness } & \multirow{2}{*}{ Substrate } & Conductance & \multicolumn{3}{c}{ Filter feeder relative abundance } \\
\cline { 4 - 6 } & & & Low & Medium & High & $\boldsymbol{\alpha}_{0}$ \\
\hline Low & Fine & Low & 0.600 & 0.390 & 0.010 & 1.54 \\
Low & Fine & Medium & 0.550 & 0.440 & 0.010 & 1.54 \\
Low & Fine & High & 0.440 & 0.550 & 0.010 & 1.54 \\
Low & Coarse & Low & 0.001 & 0.600 & 0.399 & 1.54 \\
Low & Coarse & Medium & 0.001 & 0.299 & 0.700 & 1.54 \\
Low & Coarse & High & 0.010 & 0.290 & 0.700 & 1.54 \\
Medium & Fine & Low & 0.700 & 0.299 & 0.001 & 1.54 \\
Medium & Fine & Medium & 0.700 & 0.299 & 0.001 & 1.54 \\
Medium & Fine & High & 0.500 & 0.400 & 0.100 & 1.54 \\
Medium & Coarse & Low & 0.600 & 0.390 & 0.010 & 1.54 \\
Medium & Coarse & Medium & 0.250 & 0.500 & 0.250 & 1.54 \\
Medium & Coarse & High & 0.100 & 0.400 & 0.500 & 1.54 \\
High & Fine & Low & 0.800 & 0.199 & 0.001 & 1.54 \\
High & Fine & Medium & 0.800 & 0.199 & 0.001 & 1.54 \\
High & Fine & High & 0.750 & 0.200 & 0.050 & 1.54 \\
High & Coarse & Low & 0.700 & 0.290 & 0.010 & 1.54 \\
High & Coarse & Medium & 0.300 & 0.400 & 0.300 & 1.54 \\
High & Coarse & High & 0.200 & 0.500 & 0.300 & 1.54 \\
\hline
\end{tabular}

\section{B. Data table}

[Relative abundance counts and $n$ total number of samples per parent state combination]

\begin{tabular}{lllcccc}
\hline \multirow{2}{*}{ Flashiness } & \multirow{2}{*}{ Substrate } & Conductance & \multicolumn{3}{c}{ Filter feeder relative abundance } \\
\cline { 3 - 6 } & & & Low & Medium & High & n \\
\hline Low & Fine & Low & 0 & 0 & 0 & 0 \\
Low & Fine & Medium & 0 & 0 & 0 & 0 \\
Low & Fine & High & 0 & 0 & 0 & 0 \\
Low & Coarse & Low & 0 & 0 & 0 & 0 \\
Low & Coarse & Medium & 0 & 1 & 1 & 2 \\
Low & Coarse & High & 0 & 0 & 2 & 2 \\
Medium & Fine & Low & 0 & 0 & 0 & 0 \\
Medium & Fine & Medium & 0 & 0 & 0 & 0 \\
Medium & Fine & High & 0 & 0 & 0 & 0 \\
Medium & Coarse & Low & 4 & 4 & 0 & 8 \\
Medium & Coarse & Medium & 0 & 0 & 3 & 6 \\
Medium & Coarse & High & 0 & 0 & 0 & 3 \\
High & Fine & Low & 0 & 0 & 0 & 0 \\
High & Fine & Medium & 0 & 0 & 0 & 0 \\
High & Fine & High & 0 & 0 & 0 & 0 \\
High & Coarse & Low & 1 & 1 & 2 \\
High & Coarse & Medium & 0 & 0 & 4 \\
High & Coarse & High & 0 & 1 & 2 \\
\hline
\end{tabular}


Table 2-4. "Filter feeder relative abundance" node prior conditional probability table and prior weight $\left(\alpha_{0}\right)$, data table, and posterior conditional probability table and posterior weights $\left(\alpha_{0}+n\right)$.-Continued

C. Posterior conditional probability table

[Relative abundance categories in probability units and $\alpha_{0}+n$ in equivalent data points]

\begin{tabular}{llllccc}
\hline \multirow{2}{*}{ Flashiness } & \multirow{2}{*}{ Substrate } & Conductance & \multicolumn{3}{c}{ Filter feeder relative abundance } \\
\cline { 4 - 7 } & & & Low & Medium & High & $\boldsymbol{\alpha}_{0}+\boldsymbol{n}$ \\
\hline Low & Fine & Low & 0.600 & 0.390 & 0.010 & 1.54 \\
Low & Fine & Medium & 0.550 & 0.440 & 0.010 & 1.54 \\
Low & Fine & High & 0.440 & 0.550 & 0.010 & 1.54 \\
Low & Coarse & Low & 0.001 & 0.600 & 0.399 & 1.54 \\
Low & Coarse & Medium & 0.000 & 0.413 & 0.587 & 3.54 \\
Low & Coarse & High & 0.004 & 0.126 & 0.869 & 3.54 \\
Medium & Fine & Low & 0.700 & 0.299 & 0.001 & 1.54 \\
Medium & Fine & Medium & 0.700 & 0.299 & 0.001 & 1.54 \\
Medium & Fine & High & 0.500 & 0.400 & 0.100 & 1.54 \\
Medium & Coarse & Low & 0.516 & 0.482 & 0.002 & 9.54 \\
Medium & Coarse & Medium & 0.051 & 0.102 & 0.847 & 7.54 \\
Medium & Coarse & High & 0.034 & 0.136 & 0.830 & 4.54 \\
High & Fine & Low & 0.800 & 0.199 & 0.001 & 1.54 \\
High & Fine & Medium & 0.800 & 0.199 & 0.001 & 1.54 \\
High & Fine & High & 0.750 & 0.200 & 0.050 & 1.54 \\
High & Coarse & Low & 0.587 & 0.409 & 0.004 & 3.54 \\
High & Coarse & Medium & 0.131 & 0.174 & 0.695 \\
High & Coarse & High & 0.047 & 0.271 & 0.682 & 3.54 \\
\hline
\end{tabular}


Table 2-5. "P+E relative abundance" node prior conditional probability table and prior weight $\left(\alpha_{0}\right)$, data table, and posterior conditional probability table and posterior weights $\left(\alpha_{0}+n\right)$.

\section{A. Prior conditional probability table}

[Relative abundance categories in probability units and $\alpha_{0}$ in equivalent data points; rows selected for prior weight elicitation are highlighted]

\begin{tabular}{lllcccc}
\hline \multirow{2}{*}{ Flashiness } & \multirow{2}{*}{ Substrate } & Conductance & \multicolumn{3}{c}{ P+E relative abundance } \\
\cline { 4 - 6 } & & & Low & Medium & High & $\boldsymbol{\alpha}_{0}$ \\
\hline Low & Fine & Low & 0.400 & 0.550 & 0.050 & 5.07 \\
Low & Fine & Medium & 0.700 & 0.290 & 0.010 & 5.07 \\
Low & Fine & High & 0.700 & 0.299 & 0.001 & 5.07 \\
Low & Coarse & Low & 0.010 & 0.390 & 0.600 & 5.07 \\
Low & Coarse & Medium & 0.100 & 0.600 & 0.300 & 5.07 \\
Low & Coarse & High & 0.300 & 0.600 & 0.100 & 5.07 \\
Medium & Fine & Low & 0.500 & 0.400 & 0.100 & 5.07 \\
Medium & Fine & Medium & 0.550 & 0.400 & 0.050 & 5.07 \\
Medium & Fine & High & 0.699 & 0.300 & 0.001 & 5.07 \\
Medium & Coarse & Low & 0.010 & 0.390 & 0.600 & 5.07 \\
Medium & Coarse & Medium & 0.010 & 0.600 & 0.390 & 5.07 \\
Medium & Coarse & High & 0.400 & 0.550 & 0.050 & 5.07 \\
High & Fine & Low & 0.490 & 0.500 & 0.010 & 5.07 \\
High & Fine & Medium & 0.700 & 0.290 & 0.010 & 5.07 \\
High & Fine & High & 0.700 & 0.299 & 0.001 & 5.07 \\
High & Coarse & Low & 0.400 & 0.400 & 0.200 & 5.07 \\
High & Coarse & Medium & 0.500 & 0.450 & 0.050 & 5.07 \\
High & Coarse & High & 0.600 & 0.399 & 0.001 & 5.07 \\
\hline
\end{tabular}

\section{B. Data table}

[Relative abundance counts and $n$ total number of samples per parent state combination]

\begin{tabular}{|c|c|c|c|c|c|c|}
\hline \multirow{2}{*}{ Flashiness } & \multirow{2}{*}{ Substrate } & \multirow{2}{*}{ Conductance } & \multicolumn{4}{|c|}{$\mathrm{P}+\mathrm{E}$ relative abundance } \\
\hline & & & Low & Medium & High & $n$ \\
\hline Low & Fine & Low & 0 & 0 & 0 & 0 \\
\hline Low & Fine & Medium & 0 & 0 & 0 & 0 \\
\hline Low & Fine & High & 0 & 0 & 0 & 0 \\
\hline Low & Coarse & Low & 0 & 0 & 0 & 0 \\
\hline Low & Coarse & Medium & 0 & 2 & 0 & 2 \\
\hline Low & Coarse & High & 1 & 1 & 0 & 2 \\
\hline Medium & Fine & Low & 0 & 0 & 0 & 0 \\
\hline Medium & Fine & Medium & 0 & 0 & 0 & 0 \\
\hline Medium & Fine & High & 0 & 0 & 0 & 0 \\
\hline Medium & Coarse & Low & 0 & 5 & 3 & 8 \\
\hline Medium & Coarse & Medium & 3 & 3 & 0 & 6 \\
\hline Medium & Coarse & High & 1 & 2 & 0 & 3 \\
\hline High & Fine & Low & 0 & 0 & 0 & 0 \\
\hline High & Fine & Medium & 0 & 0 & 0 & 0 \\
\hline High & Fine & High & 0 & 0 & 0 & 0 \\
\hline High & Coarse & Low & 0 & 1 & 1 & 2 \\
\hline High & Coarse & Medium & 2 & 0 & 0 & 2 \\
\hline High & Coarse & High & 4 & 1 & 0 & 5 \\
\hline
\end{tabular}


Table 2-5. "P+E relative abundance" node prior conditional probability table and prior weight $\left(\alpha_{0}\right)$, data table, and posterior conditional probability table and posterior weights $\left(\alpha_{0}+n\right)$.-Continued

C. Posterior conditional probability table

[Relative abundance categories in probability units and $\alpha_{0}+n$ in equivalent data points]

\begin{tabular}{lllcccc}
\hline \multirow{2}{*}{ Flashiness } & \multirow{2}{*}{ Substrate } & Conductance & \multicolumn{4}{c}{ P+E relative abundance } \\
\cline { 4 - 7 } & & & Low & Medium & High & $\boldsymbol{\alpha}_{0}+\boldsymbol{n}$ \\
\hline Low & Fine & Low & 0.400 & 0.550 & 0.050 & 5.07 \\
Low & Fine & Medium & 0.700 & 0.290 & 0.010 & 5.07 \\
Low & Fine & High & 0.700 & 0.299 & 0.001 & 5.07 \\
Low & Coarse & Low & 0.010 & 0.390 & 0.600 & 5.07 \\
Low & Coarse & Medium & 0.072 & 0.713 & 0.215 & 7.07 \\
Low & Coarse & High & 0.357 & 0.572 & 0.072 & 7.07 \\
Medium & Fine & Low & 0.500 & 0.400 & 0.100 & 5.07 \\
Medium & Fine & Medium & 0.550 & 0.400 & 0.050 & 5.07 \\
Medium & Fine & High & 0.699 & 0.300 & 0.001 & 5.07 \\
Medium & Coarse & Low & 0.004 & 0.534 & 0.462 & 13.07 \\
Medium & Coarse & Medium & 0.276 & 0.546 & 0.179 & 11.07 \\
Medium & Coarse & High & 0.375 & 0.593 & 0.031 & 8.07 \\
High & Fine & Low & 0.490 & 0.500 & 0.010 & 5.07 \\
High & Fine & Medium & 0.700 & 0.290 & 0.010 & 5.07 \\
High & Fine & High & 0.700 & 0.299 & 0.001 & 5.07 \\
High & Coarse & Low & 0.287 & 0.428 & 0.285 & 7.07 \\
High & Coarse & Medium & 0.641 & 0.323 & 0.036 & 7.07 \\
High & Coarse & High & 0.699 & 0.300 & 0.001 & 10.07 \\
\hline
\end{tabular}


Table 2-6. "BCG" node prior conditional probability table and prior weight $\left(\alpha_{0}\right)$, data table, and posterior conditional probability table and posterior weights $\left(\alpha_{0}+n\right)$.

\section{A. Prior conditional probability table}

[BCG tier categories in probability units and $\alpha_{0}$ in equivalent data points; rows selected for prior weight elicitation are highlighted]

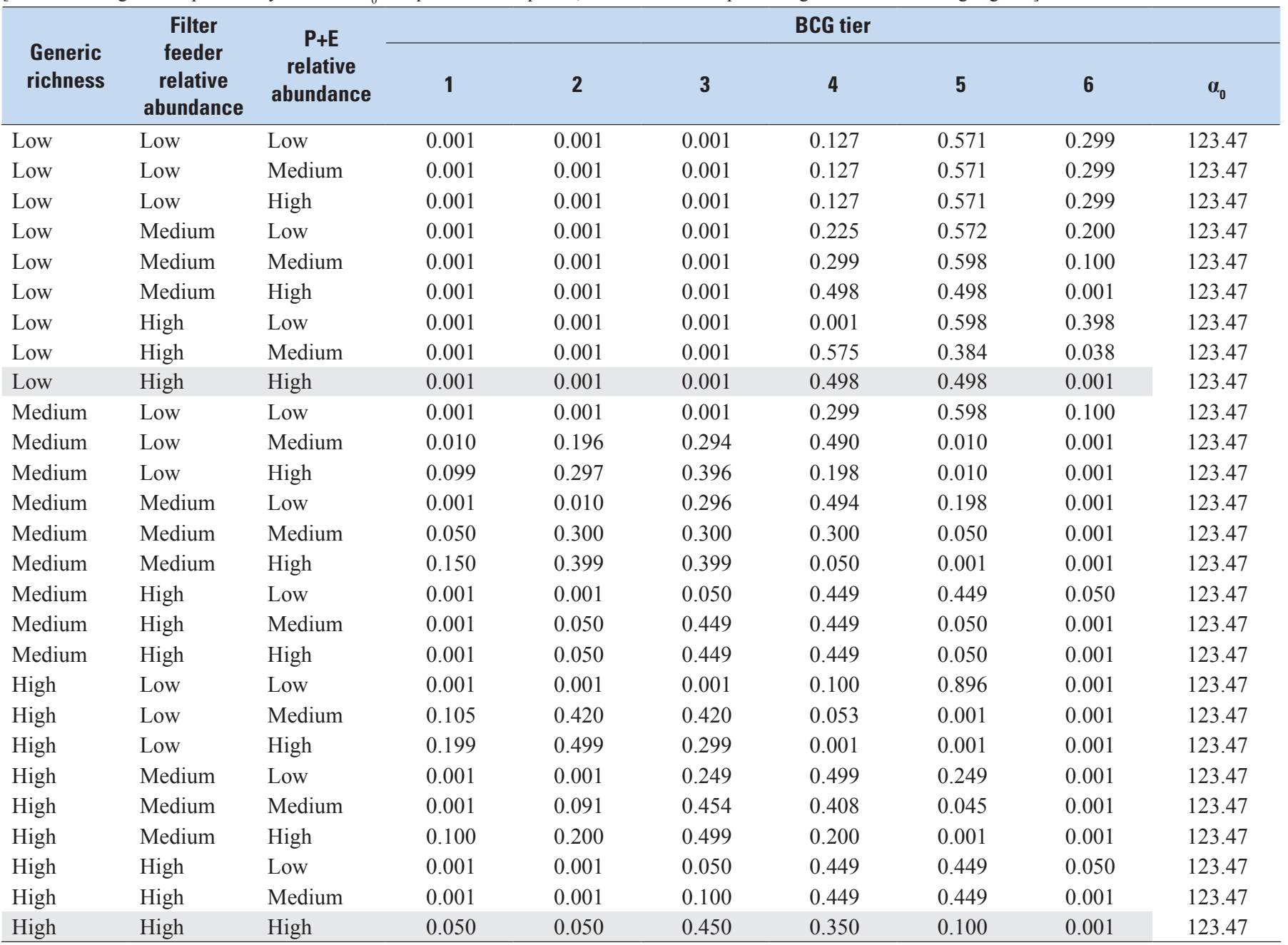


Table 2-6. "BCG" node prior conditional probability table and prior weight $\left(\alpha_{0}\right)$, data table, and posterior conditional probability table and posterior weights $\left(\alpha_{0}+n\right)$.-Continued

\section{B. Data table}

[BCG tier counts and $n$ total number of samples per parent state combination]

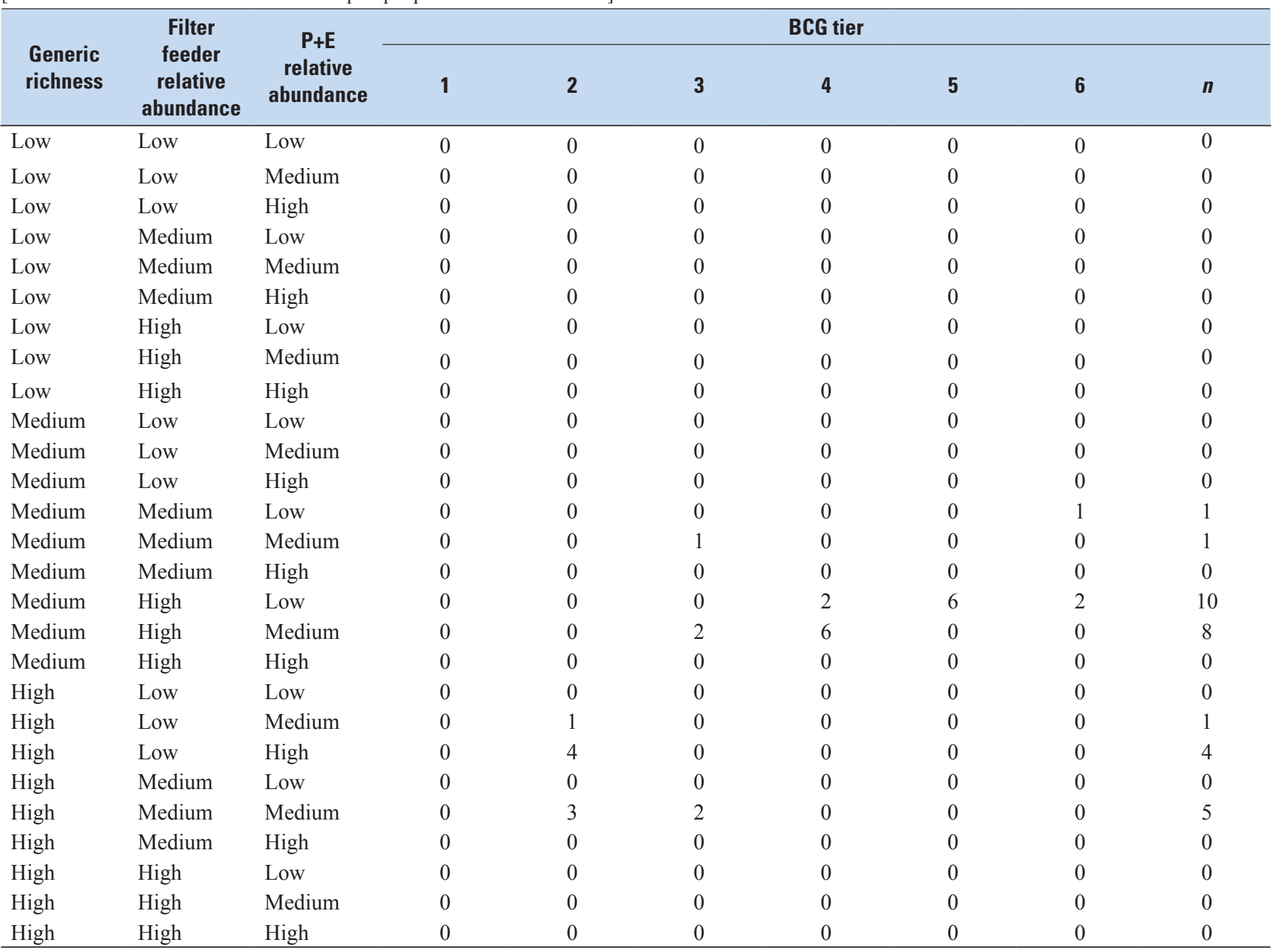


Table 2-6. "BCG" node prior conditional probability table and prior weight $\left(\alpha_{0}\right)$, data table, and posterior conditional probability table and posterior weights $\left(\alpha_{0}+n\right)$.-Continued

\section{Posterior conditional probability table}

[BCG tier categories in probability units and $\alpha_{0}+n$ in equivalent data points]

\begin{tabular}{|c|c|c|c|c|c|c|c|c|c|}
\hline $\begin{array}{l}\text { Generic } \\
\text { richness }\end{array}$ & $\begin{array}{c}\text { Filter } \\
\text { feeder } \\
\text { relative } \\
\text { abundance }\end{array}$ & $\begin{array}{c}\mathrm{P}+\mathrm{E} \\
\text { relative } \\
\text { abundance }\end{array}$ & \multicolumn{7}{|c|}{ BCG tier } \\
\hline Low & Low & Medium & 0.001 & 0.001 & 0.001 & 0.127 & 0.571 & 0.299 & 123.47 \\
\hline Low & Low & High & 0.001 & 0.001 & 0.001 & 0.127 & 0.571 & 0.299 & 123.47 \\
\hline Low & Medium & High & 0.001 & 0.001 & 0.001 & 0.498 & 0.498 & 0.001 & 123.47 \\
\hline Low & High & Low & 0.001 & 0.001 & 0.001 & 0.001 & 0.598 & 0.398 & 123.47 \\
\hline Low & High & Medium & 0.001 & 0.001 & 0.001 & 0.575 & 0.384 & 0.038 & 123.47 \\
\hline Low & High & High & 0.001 & 0.001 & 0.001 & 0.498 & 0.498 & 0.001 & 123.47 \\
\hline Medium & Low & Low & 0.001 & 0.001 & 0.001 & 0.299 & 0.598 & 0.100 & 123.47 \\
\hline Medium & Medium & Medium & 0.050 & 0.297 & 0.305 & 0.297 & 0.050 & 0.001 & 124.47 \\
\hline Medium & Medium & High & 0.150 & 0.399 & 0.399 & 0.050 & 0.001 & 0.001 & 123.47 \\
\hline Medium & High & Low & 0.001 & 0.001 & 0.046 & 0.430 & 0.460 & 0.061 & 133.47 \\
\hline Medium & High & Medium & 0.001 & 0.047 & 0.437 & 0.467 & 0.047 & 0.001 & 131.47 \\
\hline Medium & High & High & 0.001 & 0.050 & 0.449 & 0.449 & 0.050 & 0.001 & 123.47 \\
\hline High & Low & Low & 0.001 & 0.001 & 0.001 & 0.100 & 0.896 & 0.001 & 123.47 \\
\hline High & Low & Medium & 0.104 & 0.425 & 0.417 & 0.052 & 0.001 & 0.001 & 124.47 \\
\hline High & Low & High & 0.193 & 0.514 & 0.290 & 0.001 & 0.001 & 0.001 & 127.47 \\
\hline High & Medium & Low & 0.001 & 0.001 & 0.249 & 0.499 & 0.249 & 0.001 & 123.47 \\
\hline High & Medium & Medium & 0.001 & 0.111 & 0.452 & 0.392 & 0.044 & 0.001 & 128.47 \\
\hline High & Medium & High & 0.100 & 0.200 & 0.499 & 0.200 & 0.001 & 0.001 & 123.47 \\
\hline
\end{tabular}




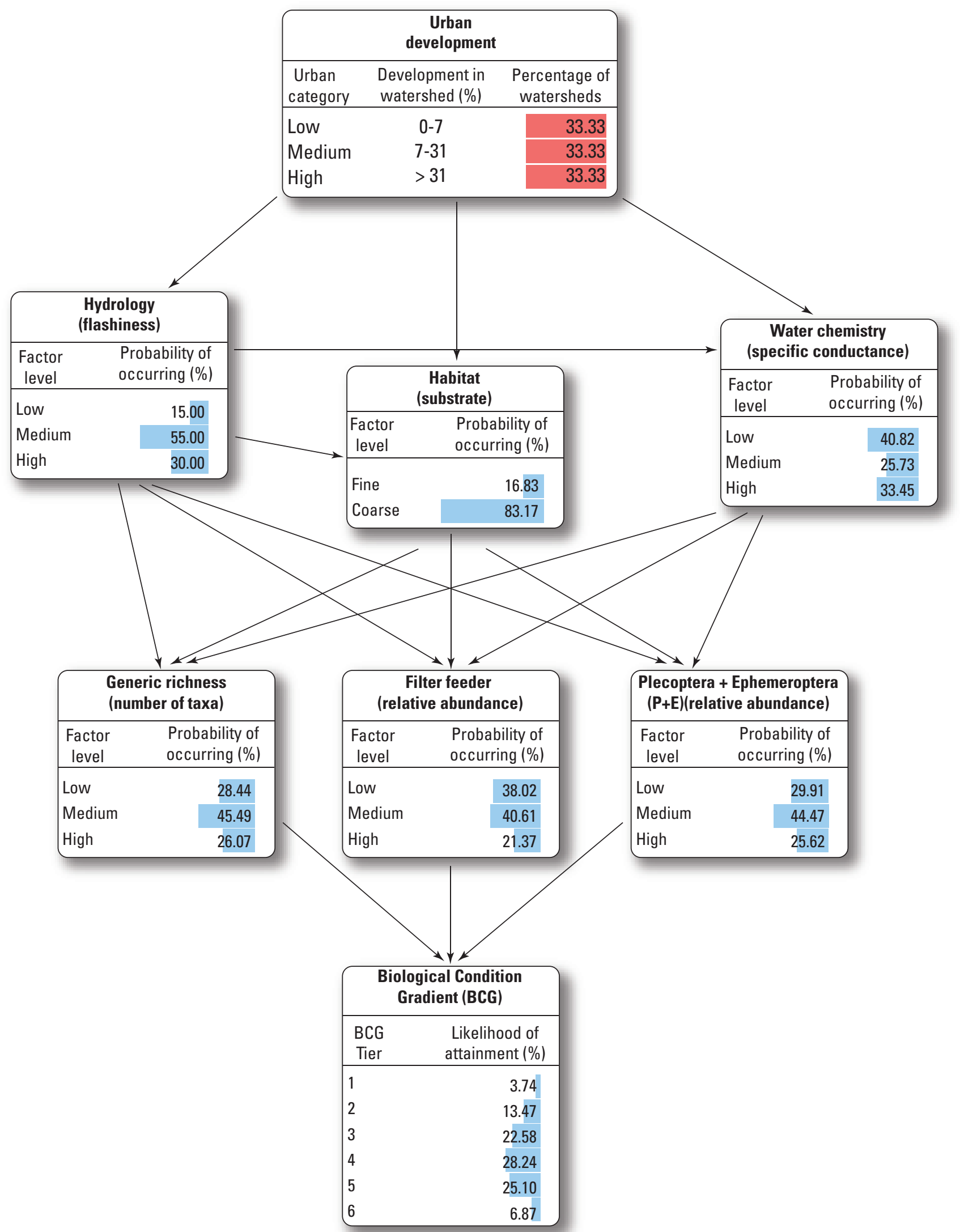

Figure 2-1. Prior Northeast Bayesian network. [Probabilities are represented as percentages, numerically and graphically (horizontal colored bars). Factor levels are defined in table 3.] 


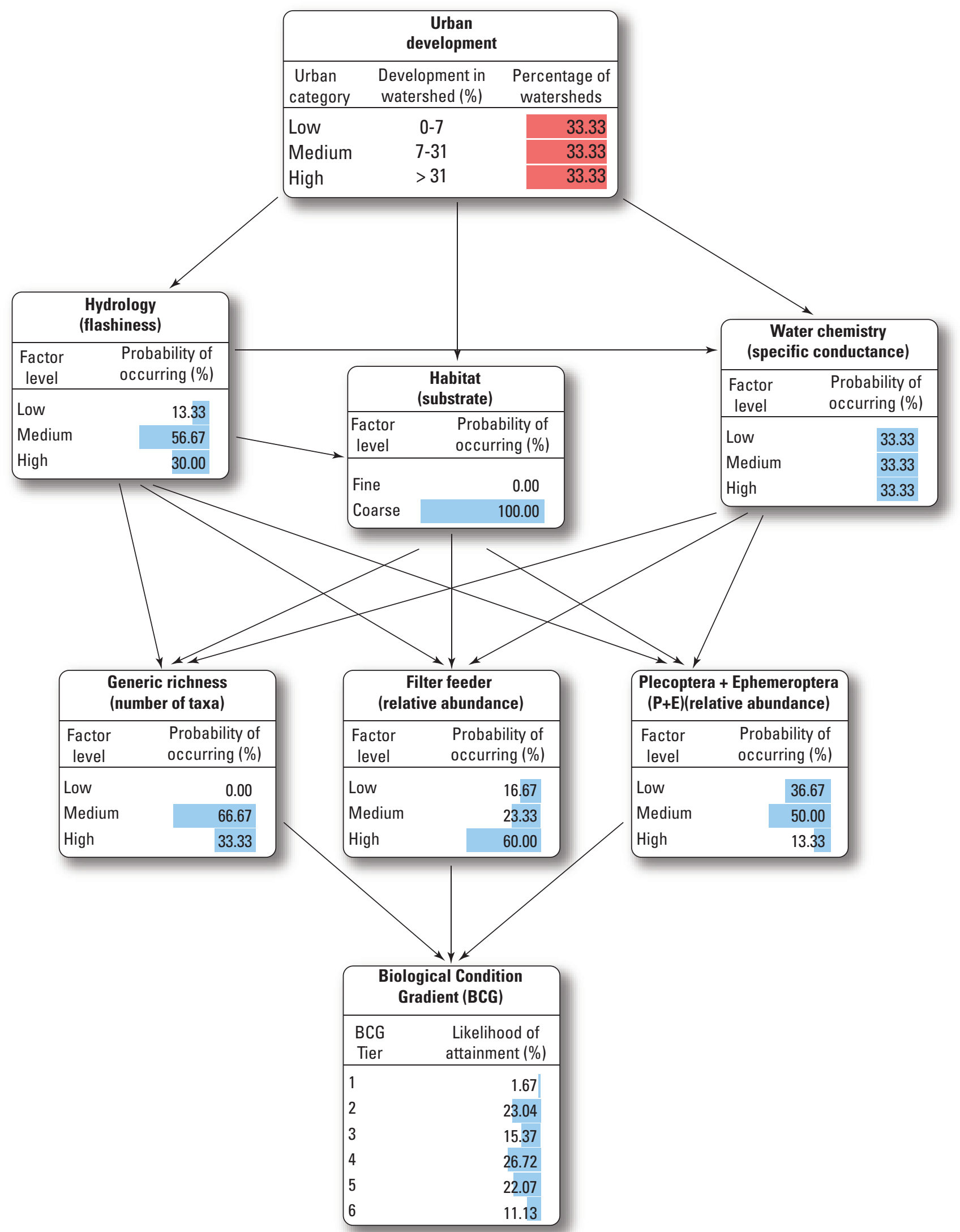

Figure 2-2. Data-only Northeast Bayesian network (uninformed prior updated with data). [Probabilities are represented as percentages, numerically and graphically (horizontal colored bars). Factor levels are defined in table 3.] 
Manuscript approved on February 3, 2012.

\section{Prepared by:}

USGS Science Publishing Network

Edited by Michael Deacon

Layout by Kay P. Naugle

For additional information regarding this publication, contact:

Roxolana O. Kashuba, Hydrologist

USGS North Carolina Water Science Center

3916 Sunset Ridge Road

Raleigh, NC 27607

phone: 919-571-4088

e-mail: rkashuba@usgs.gov

Or visit the Effects of Urbanization on Stream Ecosystems (EUSE) NAWOA Project Web site at:

http://water.usgs.gov/nawqa/urban/

A PDF version of this publication is available online at http://pubs.usgs.gov/sir/2012/5030/ 
